\title{
$F$-REGULARITY, TEST ELEMENTS, AND SMOOTH BASE CHANGE
}

\author{
MELVIN HOCHSTER AND CRAIG HUNEKE
}

\begin{abstract}
This paper deals with tight closure theory in positive characteristic. After a good deal of preliminary work in the first five sections, including a treatment of $F$-rationality and a treatment of $F$-regularity for Gorenstein rings, a very widely applicable theory of test elements for tight closure is developed in $\S 6$ and is then applied in $\S 7$ to prove that both tight closure and $F$-regularity commute with smooth base change under many circumstances (where "smooth" is used to mean flat with geometrically regular fibers). For example, it is shown in $\S 6$ that for a reduced ring $R$ essentially of finite type over an excellent local ring of characteristic $p$, if $c$ is not in any minimal prime of $R$ and $R_{c}$ is regular, then $c$ has a power that is a test element. It is shown in $\S 7$ that if $S$ is a flat $R$-algebra with regular fibers and $R$ is $F$-regular then $S$ is $F$-regular. The general problem of showing that tight closure commutes with smooth base change remains open, but is reduced here to showing that tight closure commutes with localization.
\end{abstract}

\section{INTRODUCTION}

Throughout this paper, unless otherwise specified, all rings are commutative, associative, with identity, and Noetherian, and all modules are unital and finitely generated. In [HH4] the authors introduced the notion of the tight closure for an ideal or submodule, both for Noetherian rings of positive prime characteristic $p$ and for finitely generated algebras over a field of characteristic 0 . Expository (or partially expository) accounts of the theory are given in [HH1-3], [Hul], and [Ho9]. [HH8] contains a detailed study of the notion of phantom homology (roughly speaking, homology is phantom when the cycles are in the tight closure of the boundaries). The theory of tight closure and related matters are studied further in [HH6], [HH9-11], and in [AHH], [Ab1-3], [FeW], [Gla], [Sm1-3], [Sw1,2], [Vel1,2], [W2], and [Wil1,2].

The notion of tight closure has sparked the study of rings in which every ideal is tightly closed, which are called weakly $F$-regular rings. A ring is called $F$-regular if it and all of its localizations are weakly $F$-regular.

The theory of tight closure has had a tremendous number of applications, many of them unexpected. It yields new proofs that rings of invariants of linearly reductive groups acting on regular rings are Cohen-Macaulay (cf. [HR1-2],

Received by the editors May 7, 1994.

1991 Mathematics Subject Classification. Primary 13E05, 13A35, 13B99, 13 H05.

Key words and phrases. Tight closure, $F$-regular ring, characteristic $p$, test element, smooth base change.

Both authors were supported in part by grants from the National Science Foundation. 
[Ke], [B], [HoE], and [HH4]) and a new perspective on that fact and its generalizations. (Briefly, regular rings are $F$-regular, direct summands of $F$-regular rings are $F$-regular, and $F$-regular rings are Cohen-Macaulay.) It also furnishes a new proof (see [HH4], §5) of the Briançon-Skoda theorem on integral closures of ideals in regular rings in a greatly strengthened form in the equicharacteristic case (cf. [BrS], [LS], [LT], and [Sk] for background). This theory also allows one to prove various local homological theorems (known in the equicharacteristic case and conjectured in mixed characteristic: cf. [PS1-2], [Rol-5], [Ho13], [Ho5-7], [EvG1-3], and [Du] for further information) in greatly improved forms: see [HH4], $\S 10$ and [HH8], $\S \S 4-6$, where this program is carried out. It is one of the tools utilized in [Hu3] to prove unexpectedly strong uniform Artin-Rees theorems. Moreover, the study of tight closure led to the discovery of the Cohen-Macaulay property for absolute integral closures $R^{+}$of excellent local domains $R$ in characteristic $p$ (see [HH7] and the final paragraph of this introduction), and continues to have very interesting interactions with the study of $R^{+}$.

Finally, we mention that the theory of tight closure provides a very useful handle on the problem of ccntrolling what happens when one performs operations, such as colon, intersection, product and sum, even repeatedly, on ideals generated by monomials in a system of parameters: in many situations the resulting ideal is contained in the tight closure of the "formal" or "expected" answer which one would get if the parameters were actually an $R$-sequence (or indeterminates). See $\S 7$ of [HH4] for a detailed treatment.

The greatest part of this paper is devoted to improving the theory of test elements $(\S 6)$ and then applying this improved theory to the study of smooth base change ( $(7)$. To explain our motivation, we first recall the definition of tight closure for ideals in a Noetherian ring $R$ of positive prime characteristic $p$. (See $\S 3$ for the more general definition for submodules of a module and for more detail.) Let $I \subseteq R$ be an ideal and let $x \in R$. Then we say that $x$ is in the tight closure $I^{*}$ of $I$ if there exists an element $c \in R$, not in any minimal prime, such that for all sufficiently large integers $q$ of the form $p^{e}$, where $e$ is a nonnegative integer, $c x^{q} \in I^{[q]}$, the ideal of $R$ generated by the $q$ th powers of the elements of $I$. Notice that $c$ is allowed to depend both on $x$ and $I$. In the case where $R$ is reduced (which is the main case for our purposes), one can actually choose $c$ so that $c x^{q} \in I^{[q]}$ for all $q=p^{e}, e \geq 0$. In many applications one would like to be able to choose an element $c$, independent of $x$ or $I$, so that $c$ works in all tight closure tests (i.e., $x \in I^{*}$ if and only if $c x^{q} \in I^{[q]}$ for all $q=p^{e}$ ). For simplicity, we are not discussing tight closure of modules in detail at this point, but one also wants the same element $c$ to work in all tight closure tests for modules. Such an element, if it exists, is called a test element. Furthermore, one wants $c$ to retain this property if one passes to a local ring of $R$ or even to the completion of that local ring. If $c$ has this stronger property it is called a completely stable test element. One of the main results of $\S 6$ asserts that if $R$ is reduced and essentially of finite type over an excellent local ring of characteristic $p$, then any element $c \in R$ not in a minimal prime such that $R_{c}$ is regular has a power which is a completely stable test element.

In particular, this provides an abundance of test elements for reduced ex- 
cellent local rings. We are able to conclude, for example, that if $R$ is locally excellent and weakly $F$-regular then $R$ is Cohen-Macaulay. See Theorem 6.27.

We do not know whether every excellent reduced ring of characteristic $p$ has a test element, even if we assume finite Krull dimension. Some results in low dimension are given in [Ab2].

One consequence of the theory of test elements obtained here is that if $h$ : $R \rightarrow S$ is an arbitrary homomorphism of Noetherian rings of characteristic $p$, then if $S$ has a completely stable test element or if $R$ is essentially of finite type over an excellent local ring, then tight closure is preserved by this base change (for example, if $x \in R$ is in the tight closure of $I \subseteq R$, then $h(x)$ is in the tight closure of $I S$ ). Previously, in results of this type we had to make unpleasant technical assumptions to prevent elements used in tight closure tests in $R$ from mapping into a minimal prime of $S$. For example, the results of $\S 4$ and $\S 6$ of [HH8] are greatly improved by this result.

The very natural seeming results on smooth base change obtained in $\S 7$ are highly dependent on the results of $\S 6$. The point is that when one has a homomorphism $R \rightarrow S$ it is difficult to compare tight closures over $R$ with those over $S$ unless one has a test element in $R$ that is also a test element in $S$. The key point is that when $R \rightarrow S$ is smooth and $c \in R$ is such that $R_{c}$ is regular, then $S_{c}$ is also regular. This enables us (see Theorem 6.22) to prove the existence of elements of $R$ that are test elements for both rings.

The theory of test elements has found a surprising application apart from tight closure in [Hu3], where a very strong uniform Artin-Rees theorem is proved.

A reasonably satisfactory theory concerning the behavior of tight closure under smooth base change has been obtained in $\S 7$. The reader should note that the term "smooth" here is used for flat homomorphisms $R \rightarrow S$ of Noetherian rings such that all fibers are geometrically regular: the term "regular" is often used in the literature instead. This is equivalent to the usual notion of smoothness when the $S$ is finitely presented over $R$. The main results of $\S 7$ are summarized at its beginning, in Theorems 7.1, 7.2, 7.3, and 7.4, and the reader may wish to consult them at this point. The problem of comparing the behavior of tight closure over $R$ and $S$ when one makes a smooth base change turns out to be extraordinarily difficult, even once one knows that there are "joint" test elements. This difficulty may already be seen in the fact that we do not even know whether tight closure commutes with localization in the general case. In $\S 7$ we show that, in a strong sense, the difficulty with localization is the only difficulty: see Theorems 7.1 and 7.18.

Corresponding results on base change for $F$-rational rings of characteristic $p$ (rings in which every ideal generated by parameters is tightly closed) are obtained in iVel1,2]. It is also worth noting that in [Sm1,2], [Vel1,2], and in [HH11] a notion of "test element for parameter ideals" is considered.

When $R$ has a test element, the ideal $\tau(R)$ generated by the test elements has turned out to be a very interesting invariant of $R$ (all the elements of $\tau(R)$ not in a minimal prime are test elements). We are able to prove some results about the behavior of $\tau(R)$ under certain kinds of base change for complete local rings: see, for example, Theorems 7.2 and 7.36. (The ideal $\tau(R)$ is defined whether $R$ has a test element or not: see Definition 3.7 and Proposition 3.8 further details.) 
One of the main results of [HH9] asserts that if $R$ is a locally excellent Gorenstein ring of positive prime characteristic, then $R$ is $F$-regular if and only if $R$ is a direct summand of every module-finite extension ring. (The latter condition is studied in [Ma] and in [Hol].) We want to point out that this result is highly dependent on Corollary 7.34 established here, whose proof, in turn, requires most of what comes prior to it in $\S 6$ and $\S 7$ of this paper.

One of the most persistently frustrating aspects of the theory of tight closure has been the problem of proving results on when tight closure commutes with localization, i.e., given $N \subseteq M$ and a multiplicative system $W$, when does $W^{-1}\left(N^{*}\right)=\left(W^{-1} N\right)^{*}$. In [AHH] we use the results of this paper to prove partial results in this direction under various hypotheses: one is when $M=R$ and $N$ is an ideal generated by a part of a system of parameters or by monomials in parameters. Another is for the case of ideals (or modules) expanded from a regular ring to a module-finite extension ring. A third is for pairs of modules $N \subseteq M$ such that $M / N$ has a finite phantom projective resolution in the sense of [Ab1]. The reader is referred to $[\mathrm{AHH}]$ for further information. In each instance certain additional hypotheses are needed that we shall not discuss in detail here.

What we do want to emphasize at this point is that in all these cases the arguments depend heavily on assuming that the base ring possesses a test element of some sort. Moreover, once a localization result is established, Theorem 7.18 immediately implies that there is a corresponding result for arbitrary smooth base change.

The paper is organized as follows: $\S 2$ contains certain conventions for notation and terminology, and $\S 3$ reviews briefly some definitions and facts that we need from [HH4]. In $\S 4$ we discuss issues connected with tight closure and $F$ regularity in the case of Gorenstein rings, as well as the notion of $F$-rationality (which means that parameter ideals, which are defined in the third paragraph of $\S 2$, are tightly closed; see also [FeW]). It is an open question whether, for finitely generated algebras over a field of characteristic zero, $F$-rationality is equivalent to having rational singularities. In the Gorenstein case, $F$-rational, weakly $F$-regular, and $F$-regular are all equivalent.

In $\S 5$ we review, in a strengthened form, some results about strong $F$ regularity and test elements that were obtained in [HH3] in the case where the Frobenius endomorphism is a finite morphism, and that are needed for our new study of test elements in $\S 6$.

As already mentioned, $\S 7$ contains the main results on smooth base change.

In $\S 8$ we give a proof that if $K$ is a field of characteristic $p$ of infinite transcendence degree over its prime field and $R$ is a finitely generated $K$ algebra that is weakly $F$-regular, then $R$ is $F$-regular. We are indebted to M. P. Murthy, who showed us how to prove this in the case where $K$ is uncountable, and suggested that it ought to be true under the weaker assumption given above. We also want to thank Murthy for pointing out an error in an earlier version of this paper.

This paper deals exclusively with tight closure theory in characteristic $p$. The characteristic 0 theory will be pursued in [HH10].

We conclude this introduction by mentioning one more result which gives a different perspective on tight closure. The authors have recently shown (see [HH7]; [HH5] and [Hu2] give expository accounts) that if $R$ is an excellent local 
domain of characteristic $p$, then the integral closure $R^{+}$of $R$ in an algebraic closure of the fraction field of $R$ (which is called the absolute integral closure of $R$ ) is a big Cohen-Macaulay algebra for $R$. The results of [HH9] imply that if $I$ is an ideal of $R$ then $I R^{+} \cap R \subseteq I^{*}$. So far as the authors know, it is possible that $I^{*}=I R^{+} \cap R$ in very great generality. In fact, for locally excellent domains $R$, it is true that if for every prime ideal $P$ containing $I$ the ideal $I R_{P}$ in $R_{P}$ is generated by part of a system of parameters, then $I^{*}=I R^{+} \cap R$. The general case is proved in $[\mathrm{Sm} 1,2]$. (The special case where the number of generators is at most three is done in [HH11].) One can, in any case, define a closure operation (call it, for the moment, $R^{+}$-closure) using contracted expansions from $R^{+}$. Each of the two kinds of closure suggests properties that the other kind ought to have, and this has been very useful to us in developing the theory. If the two kinds of closure do turn out to be different, then there ought to be a body of results for $R^{+}$-closure that parallels what is done here for tight closure.

\section{NOTATION AND CONVENTIONS}

In any commutative ring $R, R^{o}$ denotes the complement of the union of the set of minimal primes. Thus, if $R$ is a domain, $R^{o}=R-\{0\}$.

By a local ring $(R, m, K)$ we mean a Noetherian ring $R$ with a unique maximal ideal $m$ such that $K=R / m$ is the residue field.

A parameter in a Noetherian ring $R$ is an element of $R^{o}$. A sequence of elements $x_{1}, \ldots, x_{n}$ is called a sequence of parameters if their images form part of a system of parameters in every local ring $R_{P}$ of $R$ at a prime ideal $P$ containing the ideal $I=\left(x_{1}, \ldots, x_{n}\right) R$. In this case we refer to $I$ as an ideal generated by parameters or as a parameter ideal.

Throughout, $p$ always denotes a positive prime integer. We make the following conventions for discussing rings of positive prime characteristic $p$. We shall use $e$ to denote a variable element of the set $\mathbb{N}$ of nonnegative integers and $q$ for a variable element of the set $\left\{p^{e}: e \in \mathbb{N}\right\}$. Thus, "for all $e$ " is synonymous with "for all $e \in \mathbb{N}$ " while "for some $q$ " is synonymous with "for some $q$ of the form $p^{e}$ with $e \in \mathbb{N}$ ".

We denote by $F$ or $F_{R}$ the Frobenius endomorphism of a ring $R$ of characteristic $p$, and we denote by $F^{e}$ the $e$ th iteration of $F$, so that $F^{e}(r)=r^{q}$ where $q=p^{e}$. If $R$ is a reduced ring of characteristic $p$ we write $R^{1 / q}$ for the ring obtained by adjoining $q$ th roots of all elements of $R$. The inclusion map $R \subseteq R^{1 / q}$ is isomorphic with $F^{e}: R \rightarrow R$. We write $R^{\infty}$ for $\bigcup_{q} R^{1 / q}$. The ring $R^{\infty}$ is a chronic exception to the rule that given rings are assumed to be Noetherian.

If $I \subseteq R$ and $q=p^{e}$, then $I^{[q]}$ denotes the ideal $\left(i^{q}: i \in I\right) R$, which is also the expansion $F(I) R$ of $I$ under the Frobenius map $F: R \rightarrow R$. Note that if $T$ denotes a set of generators for $R$, then $\left\{t^{q}: t \in T\right\}$ generates $I^{[q]}$.

We shall make considerable use of the Peskine-Szpiro functors $F_{R}^{e}=F^{e}$, where $R$ has characteristic $p$ and $e \in \mathbb{N}$. For any $R$-algebra $S, S \otimes_{R-}$ gives a functor from $R$-modules to $S$-modules which takes finitely generated $R$ modules to finitely generated $S$-modules. When $S$ is $R$ itself viewed as an $R$-algebra via $F^{e}$, the $e$ th iteration of the Frobenius endomorphism, we refer to this functor as the Peskine-Szpiro or Frobenius functor: see [PS1]. Thus, $F^{e}$ is a covariant functor from $R$-modules to $R$-modules that preserves finite gen- 
eration. Note that $F^{e}(R) \cong R$, that $F^{e}\left(R^{n}\right) \cong R^{n}$ (not canonically), and that if we apply $F^{e}$ to a map $R^{n} \rightarrow R^{m}$ with matrix $\left(a_{i j}\right)$ we obtain a map $R^{n} \rightarrow R^{m}$ with matrix $\left(a_{i j}^{q}\right)$, where $q=p^{e}$. The $R$-module structure on $F^{e}(M)$ is such that $r^{\prime}(r \otimes m)=\left(r^{\prime} r\right) \otimes m$. On the other hand, $r^{\prime} \otimes(r m)=\left(r^{\prime} r^{q}\right) \otimes m$. Observe also that $F^{e}(R / I) \cong R / I^{[q]}$, where $q=p^{e}$.

There is a canonical map $M \rightarrow F^{e}(M)$ that sends $m$ to $1 \otimes m$. If $q=p^{e}$ and $w \in M$, we write $w^{q}$ for the image of $w$ in $F^{e}(M)$. With this notation, $(x+y)^{q}=x^{q}+y^{q}$ and $(r x)^{q}=r^{q} x^{q}$. Moreover, $F^{e}\left(R^{n}\right)$ may be identified with $R^{n}$ in such a way that if $w=\left(a_{1}, \ldots, a_{n}\right)$ then $w^{q}=\left(a_{1}^{q}, \ldots, a_{n}^{q}\right)$. Thus, this notation for modules is consistent with our notation for the Frobenius endomorphism of $R$.

If $N \subseteq M$, we write $N^{[q]}$ (or more precisely, $N_{M}^{[q]}$ ) for $\operatorname{Im}\left(F^{e}(N) \rightarrow F^{e}(M)\right.$ ) $\left(=\operatorname{Ker}\left(F^{e}(M) \rightarrow F^{e}(M / N)\right)\right.$ by the right exactness of $\left.\otimes\right)$. We may also characterize $N^{[q]}$ as the $R$-span in $M$ of the elements $w^{q}$ for $w \in N$. Of course, $N^{[q]}$ depends heavily on what $M$ is (or, more precisely, on what $N \rightarrow M$ is). Note that $M_{M}^{[q]}=F^{e}(M)$, and we sometimes write $M^{[q]}$ for $F^{e}(M)$. The context should make clear what is meant.

Note that if $M=R$ and $N=I \subseteq R$, then $I_{R}^{[q]}$ in this notation is the same as what was earlier described as $I^{[q]}$.

\section{TIGHT CLOSURE AND TEST ELEMENTS}

In this section we give some basic definitions and results from [HH4] that are needed throughout. All rings are assumed to be Noetherian, of characteristic $p$, and the conventions of $\S 2$ apply.

(3.1) Definition. Let $N \subseteq M$ be finitely generated $R$-modules. We say that $w \in M$ is in the tight closure $N^{*}$ (or, more precisely, $N_{M}^{*}$ ) of $N$ in $M$ if there exists an element $c \in R^{o}$ (see $\S 2$ ) such that for all sufficiently large $q=p^{e}$, $c w^{q} \in N_{M}^{[q]}$. In particular, when $N=I \subseteq R, w \in I^{*}$ means that $c w^{q} \in I^{[q]}$ for all sufficiently large $q$.

It is easy to check that the image of $N_{M}^{*} / N$ in $M / N$ is the same as the tight closure of the submodule 0 in $M / N$. This enables one to reduce many problems to studying the tight closure of the zero submodule. On the other hand, one can move in the opposite direction by mapping a finitely generated free module $G$ onto $M$, letting $H$ be the inverse image of $N$ in $G$, and studying $H^{*}$ in $G: H^{*} / H \cong N^{*} / N$ under the obvious identification $G / H \cong M / N$.

(3.2) Definition. A ring $R$ is called weakly $F$-regular if every ideal is tightly closed. $R$ is called $F$-regular if $W^{-1} R$ is weakly $F$-regular for every multiplicative system $W$ in $R$ (equivalently, if $R_{P}$ is weakly $F$-regular for every prime ideal $P$ of $R$ ). See Remark (8.4) of [HH4].

We do not know whether tight closure commutes with localization, nor even whether weakly $F$-regular implies $F$-regular. Theorems 3.3 and 3.4 contain partial information about what we do know that will be needed repeatedly in the body of the paper.

(3.3) Theorem. Let $R$ be a Noetherian ring of characteristic $p$ and let $N \subseteq M$ be R-modules.

(a) $N^{*}$ is a submodule of $M$ containing $N$ and $\left(N^{*}\right)^{*}=N^{*}$. If $N_{1} \subseteq$ $N_{2} \subseteq M$, then $N_{1}^{*} \subseteq N_{2}^{*}$. 
(b) (Irrelevance of nilpotents.) If $J$ is the nilradical of $R$, then $J M \subseteq N^{*}$. Moreover, if $N^{\prime}$ denotes the inverse image $(N+J M) / J M$ of $N$ in $M / J M$, then $N^{*}$ is the inverse image in $M$ of the tight closure $N^{\prime *}$ of $N^{\prime}$ in $M / J M$, where $N^{\prime *}$ may be computed over either $R$ or $R_{\mathrm{red}}$.

(c) If $R$ is reduced or if $\operatorname{Ann}_{R}(M / N)$ has positive height, then $x \in M$ is in $N^{*}$ if and only if there exists $c \in R^{o}$ such that $c x^{q} \in N_{M}^{[q]}$ for all $q=p^{e}$.

Proof. See [HH4], Proposition 8.5, parts (a), (b), (c), (e), and (j).

(3.4) Theorem. Let $R$ be a Noetherian ring of characteristic $p$.

(a) If $R$ is weakly $F$-regular, then every submodule of every finitely generated $R$-module is tightly closed.

(b) $R$ is weakly $F$-regular if and only if its localization at every maximal ideal is weakly F-regular.

(c) If $R$ is weakly $F$-regular then $R$ is normal. If $R$ is weakly $F$-regular and is a homomorphic image of a Cohen-Macaulay ring, then $R$ is Cohen-Macaulay.

(d) A regular ring is $F$-regular.

Proof. All references in this paragraph are to [HH4]. Part (a) follows from Proposition 8.7 and (b) is Corollary 4.15. Part (c) now follows from Theorem 7.15 (a) applied to a local ring $S$ of $R$ at a maximal ideal: $S$ will be equidimensional (since it is normal), parameters are then automatically permutable (since the local ring is catenary and equidimensional), and so if $x_{1}, \ldots, x_{n}$ is a system of parameters for $S$ we have that $\left(x_{1}, \ldots, x_{i}\right) S$ :s $x_{i+1} S \subseteq\left(\left(x_{1}, \ldots, x_{i}\right) S\right)^{*}$ (by Theorem $7.15(\mathrm{a}))=\left(x_{1}, \ldots, x_{i}\right) S$ (since is weakly $F$-regular). Finally, (d) is Theorem 4.6 (it is easily deduced from the flatness of the Frobenius endomorphism of a characteristic $p$ regular ring [Kul]).

We also recall the notions of test element and weak test element.

(3.5) Definition. Let $R$ be a Noetherian ring of characteristic $p$ and let $q^{\prime}=$ $p^{e^{\prime}}$ for some integer $e^{\prime} \in \mathbb{N}$. We say that $c \in R^{o}$ is a $q^{\prime}$-weak test element if for every finitely generated $R$-module $M$ and every submodule $N \subseteq M$, an element $x \in M$ is in $N_{M}^{*}$ if and only if $c x^{q} \in N_{M}^{[q]}$ for all $q \geq q^{\prime}$.

We say that $c$ is a locally (respectively, completely) stable $q^{\prime}$-weak test element if its image in (respectively, in the completion of) every local ring of $R$ is a $q^{\prime}$-weak test element.

If $c$ is a $q^{\prime}$-weak test element for $q^{\prime}=1$ then $c$ is called a test element. Corresponding terminology is used in the locally stable and completely stable cases.

(3.6) Proposition. Let $R$ be a local ring of characteristic $p$.

(a) If $c \in R$ is a $q^{\prime}$-weak test element for $\hat{R}$, then $c$ is a $q^{\prime}$-weak test element for $R$.

(b) If $R$ is weakly $F$-regular and has a completely stable weak test element $c$, then $\hat{R}$ is weakly $F$-regular. 
Proof. Part (a) follows from Proposition 8.13, part (c) of [HH4]. For (b), note that by Proposition 4.16 of [HH4] it suffices to check that ideals primary to the maximal ideal are tightly closed in $\hat{R}$, and this is immediate from Proposition $(6.1)(c)$.

Notice that part (a) implies that a completely stable test element is locally stable.

We next define an invariant ideal $\tau(R)$ which, when $R$ has a test element, turns out to be the ideal generated by the test elements.

(3.7) Definition. Let $R$ be a Noetherian ring of characteristic $p$. Define $\tau(R)=\bigcap_{M} \operatorname{Ann}_{R}\left(0_{M}^{*}\right)$, where $M$ runs through all finitely generated $R$-modules.

(3.8) Proposition. Let $R$ be a Noetherian ring of characteristic $p$.

(a) If $c \in R$, then $c \in \tau(R)$ if and only if whenever $N \subseteq M$ are finitely generated $R$-modules and $x \in N_{M}^{*}$ then $c x^{q} \in N_{M}^{[q]}$ for all $q$.

(b) $R$ has a test element if and only if $\tau(R)$ is not contained in any minimal prime of $R$. In this case, $\tau(R)$ is the ideal generated by the test elements, and $\tau(R) \cap R^{o}$ is the set of test elements.

Proof. This is Proposition (8.23), parts (a) and (b), of [HH4].

\section{GORENSTEIN RINGS AND $F$-RATIONAL RINGS}

(4.1) Definition. A Noetherian ring of characteristic $p$ is called $F$-rational if every ideal generated by parameters (see the third paragraph of $\S 2$ ) is tightly closed.

This terminology is introduced in [FeW]: the motivation is that if one makes the same definition for algebras of finite type over a field of characteristic 0 , there are no known examples which distinguish between $F$-rational rings and rings with rational singularities. It is shown in $[\mathrm{Sm} 1,3]$ that, under mild hypotheses, an $F$-rational ring of characteristic $p$ has pseudo-rational singularities in the sense of Lipman and Teissier [LT] and that for affine algebras over a field of characteristic zero, those of $F$-rational type have rational singularities. (The algebra $S$ has $F$-rational type if there is a finitely generated algebra $R$ over a finitely generated domain of characteristic zero $A$ over the integers such that the closed fibers of $A \rightarrow R$ are $F$-rational and $S \cong K \otimes_{A} R$ for some field $K$ containing $A$.) See also [Ab3], [Vel1,2].

A number of results in this section may also be found, in a slightly different form, in [FeW].

(4.2) Theorem. For Noetherian rings of characteristic $p$ the following hold.

(a) A weakly $F$-regular ring is $F$-rational.

(b) An F-rational ring is normal.

(c) An F-rational ring which is a homomorphic image of a Cohen-Macaulay ring is Cohen-Macaulay.

(d) A local ring $(R, m)$ which is a homomorphic image of a Cohen-Macaulay ring is $F$-rational if and only if it is equidimensional and the ideal generated by one system of parameters is tightly closed.

(e) A homomorphic image of a Cohen-Macaulay ring is $F$-rational if and only if its localization at every maximal ideal is F-rational. 
(f) A localization of an F-rational ring which is a homomorphic image of a Cohen-Macaulay ring is $F$-rational. In particular, a localization of a weakly F-regular ring which is a homomorphic image of a CohenMacaulay ring is Cohen-Macaulay.

(g) A Gorenstein ring is weakly $F$-regular if and only if it is $F$-rational.

(h) If $(R, m)$ is a local ring which is a homomorphic image of a CohenMacaulay ring and $x \in m$ is a nonzerodivisor such that $R / x R$ is $F$ rational, then $R$ is $F$-rational.

Before proving this, we establish Theorem 4.3 below. (At this point we want to note a correction to Lemma (7.10) of [HH4]. In the statement of (7.10) it should have been assumed that all minimal primes of $Q$ have the same height. This is the case in the situation where (7.10) is used (the middle of the second paragraph on p. 65 of [HH4]). We mention this here since Theorem (7.9) of [HH4] is used in proving part (d) of Theorem 4.3 below.) Cf. Proposition (6.28) for a global version of (4.3d) below.

(4.3) Theorem. Let $(R, m)$ be an equidimensional local Noetherian ring of characteristic $p$ which is a homomorphic image of a Cohen-Macaulay ring and let $x_{1}, \ldots, x_{d} \in m$ be part of system of parameters.

(a) If the ideal $\left(x_{1}, \ldots, x_{d}\right) R$ is tightly closed then so is $\left(x_{1}, \ldots, x_{i}\right) R$ for $0 \leq i \leq d$.

(b) If either the ideal $\left(x_{1}, \ldots, x_{d}\right) R$ or the ideal $\left(x_{1}, \ldots, x_{d-1}\right) R$ is tightly closed then $x_{1}, \ldots, x_{d}$ is a regular sequence in $R$.

(c) If $d=\operatorname{dim} R$ and either of the ideals $\left(x_{1}, \ldots, x_{d}\right) R$ or $\left(x_{1}, \ldots, x_{d-1}\right) R$ is tightly closed, then $R$ is Cohen-Macaulay.

(d) If $J=\left(x_{1}, \ldots, x_{d-1}\right) R$ then $J^{*}:_{R} x_{d} R=J^{*}$.

Moreover, if $d=n=\operatorname{dim} R$ and if $\left(x_{1}, \ldots, x_{n}\right) R$ is tightly closed, then so is every ideal generated by part of a system of parameters: i.e., $R$ is $F$-rational. Proof. We first prove (d). Let $u \in J^{*}:_{R} x_{d} R$. Then $x_{d} u \in J^{*}$ and so there exists $c_{0} \in R^{o}$ such that $c_{0}\left(x_{d} u\right)^{q} \in J^{[q]}$ for all large $q$. Thus, for all large $q$, we have that $c_{0} u^{q} \in J^{[q]}:_{R} x_{d}^{q} R=\left(x_{1}^{q}, \ldots, x_{d-1}^{q}\right) R:_{R} x_{d}^{q} R$. Let $c \in R^{o}$ and $q^{\prime}$ (a power of $p$ ) be as in the last statement of Theorem (7.9) of [HH4]. Then we have that for all large $q, c\left(c_{0} u^{q}\right)^{q^{\prime}} \in\left(J^{[q]}\right)^{\left[q^{\prime}\right]}$, i.e., $\left(c c_{0}^{q^{\prime}}\right) u^{q q^{\prime}} \in J^{\left[q q^{\prime}\right]}$, and this shows that $u \in J^{*}$. The other inclusion is obvious, and so (d) is established.

The proof of (a) reduces, by reverse induction on $i$, to the case where $d \geq 1$ and $i=d-1$. Let $u$ be any element in the tight closure of $J=$ $\left(x_{1}, \ldots, x_{d-1}\right) R$. Then $u \in\left(J+x_{d} R\right)^{*}=J+x_{d} R$ by hypothesis, say $u=$ $j+x_{d} r$. It follows that $u-j \in J^{*}+J=J^{*}$ and so $r \in J^{*}:_{R} x_{d} R=J^{*}$ by part (d), which we proved just above. Thus, $J^{*}=J+x_{d} J^{*}$, and the fact that $J^{*}=J$ now follows from Nakayama's lemma.

To see (b), note that the ideal $J_{i}=\left(x_{1}, \ldots, x_{i}\right) R$ is tightly closed for $0 \leq$ $i \leq d-1$ in either case. But, again by part (d), this implies that $x_{i+1}$ is not a zerodivisor modulo $J_{i}$ for $0 \leq i \leq d-1$, since $J_{i}:_{R} x_{i+1}=J_{i}^{*}:_{R} x_{i+1}=J_{i}^{*}$ (by part (d)) $=J_{i}$. Part (c) is immediate from (b).

Let $x_{1}, \ldots, x_{n} \in R$ and $y_{1}, \ldots, y_{n} \in R$ be two systems of parameters for $R$ and suppose that $\left(x_{1}, \ldots, x_{n}\right) R$ is tightly closed. To show that $\left(y_{1}, \ldots, y_{i}\right) R$ is tightly closed, it will suffice to show that $\left(y_{1}, \ldots, y_{n}\right) R$ is tightly closed. 
But $H_{m}^{n}(R) \cong \lim _{t} R /\left(x_{1}^{t}, \ldots, x_{n}^{t}\right) \cong \lim _{t} R /\left(y_{1}^{t}, \ldots, y_{n}^{t}\right)$ where the map from the term indexed by $t$ to that indexed by $t+1$ is induced by multiplication by $x_{1} \cdots x_{n}$ (respectively, by $\left.y_{1} \cdots y_{n}\right)$ and is injective. Thus, $R /\left(y_{1}, \ldots, y_{n}\right)$ injects into $R /\left(x_{1}^{t}, \ldots, x_{n}^{t}\right)$ for any sufficiently large $t$. To show that $\left(y_{1}, \ldots, y_{n}\right) R$ is tightly closed in $R$, it suffices to show that 0 is tightly closed in $R /\left(y_{1}, \ldots, y_{n}\right) R$, and, hence, to show that 0 is tightly closed in $R /\left(x_{1}^{t}, \ldots, x_{n}^{t}\right) R$. Thus, we have reduced to the case where $y_{i}=x_{i}^{t}$.

We can complete the argument by showing that if $\left(x_{1}, \ldots, x_{n}\right) R$ is tightly closed then so is $\left(x_{1}^{t}, \ldots, x_{n}^{t}\right) R$ for every $t$. But if the latter were not tightly closed then some element representing a socle element from $R /\left(x_{1}^{t}, \ldots, x_{n}^{t}\right) R$ must be in its tight closure, and this element will have the form $\left(x_{1} \cdots x_{n}\right)^{t-1} u$, where $u$ represents an element of the socle in $R /\left(x_{1}, \ldots, x_{n}\right)$. But then $c\left(\left(x_{1} \cdots x_{n}\right)^{t-1} u\right)^{q} \in\left(\left(x_{1}^{t}, \ldots, x_{n}^{t}\right) R\right)^{[q]}$ implies that

$$
c u^{q} \in\left(x_{1}^{q t}, \ldots, x_{n}^{q t}\right) R:_{R}\left(x_{1} \cdots x_{n}\right)^{q t-q}=\left(x_{1}^{q}, \ldots, x_{n}^{q}\right) R
$$

(since the $x_{i}$ are a regular sequence), and so, if this holds for all $q \gg 0$, then $u$ is in the tight closure of $\left(x_{1}, \ldots, x_{n}\right) R$, a contradiction.

(4.4) Remark. More generally, the argument shows that if $R$ is Noetherian of characteristic $p$ and $x_{1}, \ldots, x_{d}$ is a sequence of elements such that $I=$ $\left(x_{1}, \ldots, x_{d-1}\right) R$ or $\left(x_{1}, \ldots, x_{d}\right) R$ is tightly closed, then the sequence is an $R$-sequence provided we know that:

(i) $\left(\left(x_{1}, \ldots, x_{i}\right) R\right)^{*}:_{R} x_{i+1} R \subseteq\left(\left(x_{1}, \ldots, x_{i}\right) R\right)^{*}$ for relevant values of $i$, and

(ii) Nakayama's lemma can be applied.

There are many variant situations in which (i) holds. See, for example, Proposition 6.28 .

Proof of (4.2). (a) is immediate from the definitions. (b) follows by the same argument used to prove that weakly $F$-regular rings are normal: see (5.10) and (5.11) of [HH4]. Similarly, (c) follows from the same argument used to prove that weakly $F$-regular rings are Cohen-Macaulay: note that (b) implies that the ring is normal and so locally equidimensional. (Any ideal of height $n$ contains a sequence of elements $x_{1}, \ldots, x_{n}$ such that for $1 \leq i \leq n, x_{1}, \ldots, x_{i}$ consists of parameters. Then $\left(x_{1}, \ldots, x_{i-1}\right) R:_{R} x_{i} R \subseteq\left(\left(x_{1}, \ldots, x_{i-1}\right) R\right)^{*}$ by Theorem 7.15 of [HH4] and this ideal is equal to $\left(x_{1}, \ldots, x_{i-1}\right) R$ by the definition of $F$-rationality.)

The second condition in (d) is obviously necessary, and it is sufficient by Theorem 4.3.

To prove (e) first suppose that $R_{m}$ is $F$-rational for every maximal ideal $m$. Suppose that $\left(x_{1}, \ldots, x_{n}\right) R$ has height $n$ in $R$ and that $y$ is in its tight closure but not in the ideal. Then all this can be preserved while localizing at a suitable maximal ideal, giving a contradiction. Thus, $R$ is $F$-rational.

Now suppose that $R$ is $F$-rational, and let $P$ be any prime ideal of $R$. Choose $x_{1}, \ldots, x_{n} \in P$ to be an $R$-sequence, where $n$ is the height of $P$ (which is the same as the depth of $R$ on $P$ ). The images $x_{1} / 1, \ldots, x_{n} / 1$ in $R_{P}$ will be a system of parameters. If we can show that the ideal $\left(x_{1} / 1, \ldots, x_{n} / 1\right) R_{P}$ is tightly closed in $R_{P}$, it will follow from (4.3) that $R_{P}$ is $F$-rational. This follows from Theorem 4.5 below. 
It now follows that $R$ is $F$-rational iff all its localizations at primes are, and this implies that every localization of $R$ is $F$-rational.

We next consider $(\mathrm{g})$. If $R$ is Gorenstein, both its $F$-rationality and weak $F$-regularity can be checked locally at maximal ideals. We need only show that if $(R, m)$ is local Gorenstein and every system of parameters generates a tightly closed ideal, then every ideal is tightly closed. It suffices to prove this for $m$-primary ideals. But if $I$ is $m$-primary $R / I$ can be embedded in a finite direct sum of copies of the injective hull of $K=R / m$, and each of these can be replaced by a copy of $R /\left(x_{1}^{t}, \ldots, x_{n}^{t}\right)$ for sufficiently large $t$ (where $x_{1}, \ldots, x_{n}$ is any fixed system of parameters). Since 0 is tightly closed in each $R /\left(x_{1}^{t}, \ldots, x_{n}^{t}\right)$, this is also true in the direct sum and hence in every submodule of the direct sum.

Finally, we consider (h). Since $R / x R$ is a homomorphic image of a CohenMacaulay ring and is $F$-rational, it is C-M. Hence, $R$ is C-M and so equidimensional. Extend $x$ to a system of parameters $x, x_{1}, \ldots, x_{d}$ for $R$. It will suffice to show that these elements generate a tightly closed ideal. Suppose $c \in R^{o}$ and $c u^{q} \in\left(x^{q}, x_{2}^{q}, \ldots, x_{d}^{q}\right) R$ for all $q \gg 0$. Write $c=d x^{t}$ where $d$ is not in $x R$. Note that $x R$ is a prime ideal and that $d$ represents a nonzero element of the domain $R / x R$. If $c u^{q} \in\left(x^{q}, x_{2}^{q}, \ldots, x_{d}^{q}\right) R$ and $q>t$ we have $d u^{q} \in\left(x^{q-t}, x_{2}^{q}, \ldots, x_{d}^{q}\right) R$ for $q \gg 0$ and reducing modulo $R x$ we obtain that the image of $u$ is in the tight closure in $R / x R$ of $\left(x_{2}, \ldots, x_{d}\right)(R / x R)$. It follows that the image of $u$ is in this ideal and so $u$ is in the ideal $\left(x, x_{2}, \ldots, x_{d}\right) R$.

As mentioned in the Introduction, the next result is generalized to parameter ideals (and in other directions) in [AHH]. However, these generalizations require some assumptions on the ring (such as the existence of test elements) that are not needed in case the elements from a regular sequence.

(4.5) Theorem. Let $R$ be a Noetherian ring of characteristic $p$ and let $x_{1}, \ldots$, $x_{n}$ be an $R$-sequence. Let $S$ be a multiplicative system and let

$$
I=\left(x_{1}, \ldots, x_{n}\right) R .
$$

Then $I^{*} S^{-1} R=\left(I S^{-1} R\right)^{*}$. In particular, if $I$ is tightly closed, then $I S^{-1} R$ is tightly closed.

Before giving the proof, we prove the following:

(4.6) Lemma. Let $I$ be an ideal of an arbitrary Noetherian ring $R$ and let $S \subseteq R$ be a multiplicative system.

(a) There is an element $s \in S$ such that $\bigcup_{w \in S} I^{m}:_{R} w=I^{m}:_{R} s^{m}$ for all $m \in \mathbb{N}$.

(b) Suppose that $R$ has characteristic $p$ and $I$ is generated by a regular sequence $x_{1}, \ldots, x_{n}$. Then with $s$ as in part $(\mathrm{a}), \bigcup_{w \in S}{ }^{[q]}: w=$ $I^{[q]}: s^{(n+1) q}$ for all $q=p^{e}$.

Proof. (a) is well known but we sketch a brief argument. Choose $s$ such that, if $T=\operatorname{gr}_{I} R$, then $\operatorname{Ann}_{T} s=\operatorname{Ann}_{T} w s$ for all $w \in S$. Suppose $w$ kills $u$ modulo $I^{m}$, and $u \in I^{r}$ but $u \notin I^{r+1}, r<m$. Then $s u$ is in $I^{r+1}$. Repeating this argument $m$ times shows that $s^{m} u$ is in $I^{m}$. 
We now consider (b). Fix $q$ and an element $u \in I^{[q]}: w$ for some $w \in S$. We shall prove by induction on $h \in \mathbb{N}$ that $d_{h}=s^{q+h} u \in I^{[q]}+I^{q+h}$. This will suffice, for when $h=q n$ this yields $s^{(n+1) q} u \in I^{[q]}+I^{q(n+1)} \subseteq I^{[q]}$ when $I$ has $n$ generators.

If $h=0$ we are simply asserting that $s^{q} u \in I^{[q]}+I^{q}=I^{q}$, and this follows from the property of $s$ in part (a).

Now suppose that we have established that $d_{h} \in I^{[q]}+I^{q+h}$ for a given $h \geq 0$. Say $d_{h}=\sum_{i} r_{i}^{\prime} x_{i}^{q}+\sum_{\nu} r_{\nu} x^{\nu}$ where $\nu$ runs through $n$-tuples of nonnegative integers $\left(\nu_{1}, \ldots, \nu_{n}\right)$ such that every $\nu_{i}<q$ and the sum of the $\nu_{i}$ is $q+h$. Some $w \in S$ multiplies this element into $I^{[q]}$, giving an equation:

$$
\sum_{i} r_{i}^{\prime \prime} x_{i}^{q}=\sum_{i} w r_{i}^{\prime} x_{i}^{q}+\sum_{\nu} w r_{\nu} x^{\nu} \quad \text { or } \quad \sum_{i} a_{i} x_{i}^{q}+\sum_{\nu} w r_{\nu} x^{\nu}=0,
$$

where $a_{i}=w r_{i}^{\prime}-r_{i}^{\prime \prime}$. Since the $x_{j}$ form a regular sequence and none of the monomials occurring is formally in the ideal generated by the others, each $w r_{\nu}$ must be in $I$. But then $s r_{\nu}$ is in $I$ for each $\nu$, and so $d_{h+1}=s d_{h}=$ $\sum_{i} s r_{i}^{\prime} x_{i}^{q}+\sum_{\nu} s r_{\nu} x^{\nu}:$ the first term is in $I^{[q]}$, while the second is in $I^{q+(h+1)}$.

We note that in part (b) it is not necessary to assume characteristic $p$ nor to assume that $q$ is a prime power, if one agrees that $I^{[q]}$ simply denotes $\left(x_{1}^{q}, \ldots, x_{n}^{q}\right) R$. However, $I^{[q]}$ then depends on the choice of generators for $I$, not just on what $I$ itself is.

Proof of (4.5). We note that $I^{*} S^{-1} R \subseteq\left(I S^{-1} R\right)^{*}$ for any ideal $I$. The hard part is to show that $\left(I S^{-1} R\right)^{*} \subseteq I^{*} S^{-1} R$. If not, we can choose an element $u / 1$ in $\left(I S^{-1} R\right)^{*}$ but not in $I^{*} S^{-1} R$.

Now the statement that $u / 1 \in\left(I S^{-1} R\right)^{*}$ means that for some $c \in R^{o}$ and for all $q \gg 0$ we can choose $s(q) \in S$ such that $s(q) c u^{q} \in I^{[q]}$. (A priori, we only know that $c$ is not in any minimal prime of $R$ disjoint from $S$. However, we can modify $c$ by adding to it an element in all the minimal primes of $R$ which meet $S$ and precisely those minimal primes not meeting $S$ to which $c$. does not belong, and then raising the sum to a power. The element we are adding is nilpotent when mapped to $S^{-1} R$.)

With $s$ as in (4.6a) we then have that $s^{(n+1) q} c u^{q}=c\left(s^{n+1} u\right)^{q} \in I^{[q]}$, and then $s^{n+1} u \in I^{*}$, so that $u / 1 \in I^{*} S^{-1} R$. This completes the proof for $(4.5)$ and, at last, for (4.2).

We obtain some important corollaries by specializing to the case where the ring is Gorenstein.

(4.7) Corollary. Let $R$ be a Noetherian ring of characteristic $p$ and suppose that $R$ is Gorenstein.

(a) $R$ is $F$-rational if and only if $R$ is weakly $F$-regular if and only if $R$ is $F$-regular.

(b) If $(R, m)$ is local, then $R$ is $F$-regular if and only if the ideal generated by a single system of parameters is tightly closed.

(c) If $(R, m)$ is local, $x$ is a nonzerodivisor in $R$, and $R / x R$ is $F$-regular, then $R$ is $F$-regular.

Proof. (a) follows because the property of being Gorenstein and $F$-rational is preserved by localization. (b) and (c) are immediate from (a) and (4.2). 
(4.8) Remark. $F$-regular Gorenstein rings have a number of good properties that distinguish them from other $F$-regular rings-at least in terms of what we can prove about them. In the next section we shall see that if $R$ is a reduced Noetherian ring of characteristic $p$ such that $R^{1 / p}$ is module-finite over $R$, and $R$ is a weakly $F$-regular Gorenstein ring, then $R$ is strongly $F$-regular in the sense defined in that section. In [HH9] we show that a characteristic $p$ Gorenstein ring is $F$-regular if and only if it is a direct summand, as a module over itself, of every module-finite extension ring.

Finally, we note:

(4.9) Proposition. Let $R$ be a weakly $F$-regular ring that is a homomorphic image of Cohen-Macaulay ring. Suppose that $R_{P}$ is Gorenstein for every prime $P$ which is not maximal. Then $R$ is $F$-regular.

Proof. The localization of $R$ at every maximal ideal is weakly $F$-regular. It suffices to show that the localization at every nonmaximal prime $P$ is weakly $F$-regular. But $R_{P}$ is Gorenstein and $F$-rational, and so $F$-regular.

(4.10) Remark. By results of Watanabe there exist two-dimensional normal (hence, Cohen-Macaulay) domains $R$ finitely generated over a field such that (1) $R$ is $F$-rational but not $F$-regular (nor even $F$-pure) (see [W1]) and such that (2) $R$ is $F$-rational and $F$-pure but not weakly $F$-regular (see [W2]). The example in (2) is not Gorenstein ( $F$-rational Gorenstein rings are $F$-regular). These examples are also discussed in some detail in $\S 7$ of [HH9].

\section{STRONGLY $F$-REgUlAR RINGS AND TEST ELEMENTS}

In this section we survey the characteristics of a property of rings of characteristic $p$ that is, on the face of it, stronger than $F$-regularity but that may be equivalent to it, or even to weak $F$-regularity (there is evidence of this in low dimension in [Will,2]). This property, strong $F$-regularity, is only defined for reduced rings $R$ such that $R^{1 / p}$ is module-finite over $R$ : since the latter condition is satisfied by finitely generated algebras over a perfect field $K$ and by complete local rings $R$ with perfect residue class field $K$ (in fact, in both cases, one only needs that $K^{1 / p}$ be finite over $K$ ), it is not unduly restrictive.

Throughout the rest of this section, unless otherwise specified, $R$ denotes a reduced Noetherian ring of positive prime characteristic $p$ such that $R^{1 / p}$ is module-finite over $R$ (although we often repeat this hypothesis in stating theorems). Of course, $R^{1 / q}$ is then module-finite over $R$ for all $q=p^{e}$. For the most part, we are summarizing the results of $\S 3$ of [HH3], and so most proofs are omitted. We should note two points: first, in many instances, the rings in [HH3] were assumed to be domains, when, in fact, it is only necessary to assume that the ring be reduced. Second, the result on test elements in Theorem 5.10 , which is quite critical in obtaining the results of $\S 6$, is strengthened from what is asserted in [HH3], Theorem 3.4, in three ways:

(i) One shows that the elements $c$ discussed are test elements for modules, not just for ideals.

(ii) One can conclude that $c$ is in $\tau(R)$ whether or not $c$ is in $R^{o}$ : thus, there is no need to assume the $c \in R^{o}$.

(iii) $R$ need only be reduced, not necessarily a domain. 
However, the proof is omitted, except for some brief remarks, since it is the same as that given for Theorem 3.4 of [HH3].

(5.1) Definition. We say that a reduced Noetherian ring $R$ such that $R^{1 / p}$ is module-finite over $R$ is strongly $F$-regular if for every $c \in R^{o}$ there exists $q$ such that the $R$-linear map $R \rightarrow R^{1 / q}$ that sends 1 to $c^{1 / q}$ splits as a map of $R$-modules, i.e., iff $R c^{1 / q} \subseteq R^{1 / q}$ splits over $R$.

(5.2) Discussion. In rings that are not reduced we impose the condition that $R \stackrel{F}{\longrightarrow} R$ be module-finite instead (an equivalent condition is that $R$ be modulefinite over its subring $F(R)=R^{p}=\left\{r^{p}: r \in R\right\}$ ). (Rings satisfying this condition are sometimes called " $F$-finite.") However, the splitting condition we are studying implies that $R$ is reduced. So long as we are focusing on this condition, we might as well work with the isomorphic inclusion map $R \rightarrow R^{1 / p}$.

(5.3) Remark. Suppose that $R$ is reduced and that we do not know that $R^{1 / p}$ is module-finite over $R$. Parts of the theory developed below generalize if we change the definition of "strong $F$-regularity" to the statement that for every $c \in R^{o}$ there exists $q$ such that the $R$-linear map $R \rightarrow R^{1 / q}$ which sends 1 to $c^{1 / q}$ is pure as a map of $R$-modules (a map is pure if it is injective and remains so after tensoring with any third $R$-module). But we have chosen not to pursue this idea in detail at present.

A different generalization, "strong $F$-rationality" is studied in [Vel1,2].

(5.4) Remarks. (a) The issue of whether a homomorphism of finitely generated modules over a Noetherian ring splits is local and is unaffected by a faithfully flat extension of the base ring (since the question can be translated into whether a certain map of Hom's is onto: see [Hol]).

(b) If $R \subseteq S$ and $f: R \rightarrow M$ is split by $g$, where $M$ is an $S$-module, then $R \subseteq S$ splits: send $s$ to $g(s f(1))$.

(c) In the definition above, if a splitting exists for one choice of $c \in R^{o}$ and $q$ then $R \subseteq R^{1 / q^{\prime}}$ splits for every $q^{\prime}$. (It suffices to split $R \subseteq R^{1 / p}$ and hence $R \subseteq R^{1 / q}:$ now use (b).)

(d) Note also that if $R \rightarrow R^{1 / q}$ sending 1 to $c^{1 / q}$ splits for one choice of $q$, the map $R \rightarrow R^{1 / q^{\prime}}$ sending 1 to $c^{1 / q^{\prime}}$ splits for every $q^{\prime} \geq q$ : the map $R \rightarrow R^{1 / q}$ described is isomorphic to the map $R^{q / q^{\prime}} \rightarrow R^{1 / q^{\prime}}$ sending 1 to $c^{1 / q^{\prime}}$ and so that map splits over $R^{q / q^{\prime}}$, and this splitting may be composed with $R$-splitting $R \subseteq R^{q / q^{\prime}}$ whose existence we showed in (c).

The following result exhibits a number of the good properties of strong $F$ regularity.

(5.5) Theorem. Let $R$ be a reduced Noetherian ring of characteristic $p$ such that $R^{1 / p}$ is module-finite over $R$.

(a) $R$ is strongly $F$-regular iff $R_{P}$ is strongly $F$-regular for every prime (respectively, for every maximal) ideal $P$ of $R$. Hence, if $R$ is strongly $F$-regular, so is $W^{-1} R$ for every multiplicative system $W$.

(b) If $S$ is faithfully flat over $R$ and strongly $F$-regular then so is $R$.

(c) If $R$ is regular, then $R$ is strongly $F$-regular.

(d) If $R$ is strongly $F$-regular, then $R$ is F-regular. In particular, $R$ is normal, and is a finite product of domains, each of which is strongly 
F-regular. Conversely, a finite product of strongly F-regular rings is strongly $F$-regular.

(e) If $R^{\prime}$ is strongly $F$-regular and $R$ is a direct summand of $R^{\prime}$ as an $R$ module, then $R$ is strongly $F$-regular. In particular, a direct summand (as a module over itself) of a regular ring is strongly $F$-regular.

(f) If $R$ is weakly $F$-regular and Gorenstein, then $R$ is strongly $F$-regular.

Proof. The argument is essentially the same as the proof of Proposition 3.1 of [HH3], and is omitted.

In [Will,2] it is shown that a reduced weakly $F$-regular ring $R$ of dimension at most three such that $R^{1 / p}$ is module-finite over $R$ is strongly $F$-regular.

(5.6) Remark. The argument for part (f) in [HH3] actually shows again that a Gorenstein local ring is strongly $F$-regular provided the ideal generated by a single system of parameters is tightly closed. We have already seen ( $\$ 4)$ that a Gorenstein local ring is $F$-regular (not just weakly $F$-regular) if the ideal generated by one system of parameters is tightly closed: it is not necessary that $R^{1 / p}$ be module-finite over $R$.

(5.7) Remark. When $R^{1 / p}$ is module-finite over the reduced ring $R$ we can always choose $c \in R^{o}$ such that $\left(R^{1 / p}\right)_{c} \cong\left(R_{c}\right)^{1 / p}$ is free over $R_{c}$ : for such a $c, R_{c}$ is regular and, hence, strongly $F$-regular.

(5.8) Remark. Suppose that $R^{1 / p}$ is module-finite over the reduced ring $R$ and that $c \in R^{o}$ is such that $R_{c}$ is strongly $F$-regular. Then for every $d \in R^{o}$ there is an integer $q=p^{e}$, an integer $t \geq 0$ and an $R$-linear map $R^{1 / q} \rightarrow R$ that sends $d^{1 / q}$ to $c^{t}$. To see this, choose $q$ sufficiently large that there is an $R_{c}$-linear map $g:\left(R^{1 / q}\right)_{c} \rightarrow R_{c}$ such that $g\left(d^{1 / q}\right)=1$. Since $R^{1 / q}$ is modulefinite over $R, c^{t} g\left(R^{1 / q}\right) \subseteq R$ for sufficiently large $t$, and then $c^{t} g$, restricted to $R^{1 / q}$, has the required property. Notice that we may replace $t$ by any larger integer: in particular, we may assume that it is a power of $p$.

(5.9) Theorem. Let $R$ be a reduced Noetherian ring of characteristic $p$ such that $R^{1 / p}$ is module-finite over $R$.

(a) Let $c$ be any element of $R^{o}$ such that $R_{c}$ is strongly $F$-regular (such elements always exist). Then $R$ is strongly $F$-regular if and only if there exists $q=p^{e}$ such that the $R$-module inclusion $R c^{1 / q} \subseteq R^{1 / q}$ splits over $R$.

(b) The set $\left\{P \in \operatorname{Spec} R: R_{P}\right.$ is strongly $F$-regular $\}$ is Zariski open in $\operatorname{Spec} R$.

Proof. The argument is the same as for Theorem 3.3 of [HH3].

The constructions of test elements given in [HH4] provide only a very limited class. One of the pleasant consequences of the theory of strong $F$-regularity is that one can use it to show that every $R$ such that $R^{1 / p}$ is module-finite over $R$ has a test element and, in fact, an abundance of test elements: every element in the ideal which defines the locus of primes $P$ where $R_{P}$ is not strongly $F$-regular has a power which is a test element. In particular, in the case of an isolated local singularity, there is a power of the maximal ideal all of whose elements that are in $R^{o}$ are test elements. This follows from: 
(5.10) Theorem. Let $R$ be a reduced Noetherian ring of characteristic $p$ such that $R^{1 / p}$ is module-finite over $R$. Then every element $c^{\prime}$ such that $R_{c^{\prime}}$ is strongly $F$-regular has a power $c$ which is in $\tau(R)$ (which in this case is the ideal generated by the test elements). One can always choose such an element $c^{\prime}$ such that $c^{\prime} \in R^{o}$ and $R_{c^{\prime}}$ is regular.

More precisely, $c^{\prime}$ has a power $c$ such that there is an R-linear map $h$ of $R^{1 / p}$ to $R$ which sends 1 to $c$, and for $c \in R^{o}$ with this property such that $R_{c}$ is strongly F-regular, $c^{3}$ is in $\tau(R)$.

What is more, if $c$ has the properties that $R_{c}$ is regular and that there is an $R$-linear map $h$ of $R^{1 / p}$ to $R$ that sends 1 to $c$, then this situation is preserved when one takes the image of $c$ in either a local ring $S$ of $R$ or in the completion of $S$. It follows that every element $c^{\prime} \in R^{o}$ such that $R_{c^{\prime}}$ is regular has a power $c$ such that $c$ is a completely stable test element for $R$.

Proof. The proof of the first assertion in the second paragraph is the same as for the corresponding statement in the proof of Theorem 3.4 in [HH3]. In order to check that $c^{3}$ is a test element it suffices to consider the case of pairs of modules $N \subseteq G \cong R^{t}$. With this remark, the second assertion in the second paragraph then follows exactly the lines of the argument given in the proof of Theorem 3.4 in [HH3].

The last statement in the first paragraph follows from (5.7). This shows that there is a test element for $R$, and this implies that $\tau(R)$ is the ideal generated by the test elements (which then agrees with $\tau(R) \cap R^{o}$ ). The assertions in the second paragraph then imply all the assertions in the first paragraph.

The statements in the last paragraph are then clear: note that since $R^{1 / p}$ is module-finite over $R$, we have from [Ku2] that $R$ is excellent, and so the fibers of the map $S \rightarrow \hat{S}$ are regular. This enables us to show that $(\hat{S})_{c^{\prime}}$ is regular whenever $R_{c^{\prime}}$ is regular.

\section{TEST ELEMENTS IN FINITELY GENERATED ALGEBRAS OVER EXCELLENT LOCAL RINGS}

The main results of this section are Theorem 6.1 and Theorem 6.2 immediately below, Theorem 6.21 and Theorem 6.22 (which yield common test elements), and Theorem 6.24, which shows that, under mild conditions on $R$ or $S$, tight closure is preserved by arbitrary base change, $R \rightarrow S$, without hypotheses concerning whether $R^{o}$ or test elements of $R$ map into $S^{o}$. The conditions require that at least one of the rings $R, S$ have "sufficiently many sufficiently good" test elements. For example, it suffices if $S$ has a completely stable (weak) test element, or if $R$ is essentially of finite type over an excellent local ring.

(6.1) Theorem. Let $R$ be a ring of characteristic $p$.

(a) Suppose that $(R, m, K)$ is local ring such that $R \rightarrow \hat{R}$ has regular fibers and let $c \in R^{o}$ be any element such that $\left(R_{\mathrm{red}}\right)_{c}$ is regular. Then $c$ has a power which is a completely stable $q^{\prime}$-weak test element for $R$. If $R$ is reduced, then $c$ has a power which is a completely stable test element for $R$.

In particular, if $R$ is an excellent local ring, then $R$ has a completely stable $q^{\prime}$-weak test element; if, moreover, $R$ is reduced, then $R$ has a completely stable test element. 
(b) Let $R$ be an algebra of finite type over a local ring $(B, m, K)$ such that $B \rightarrow \hat{B}$ has smooth fibers (e.g., such that $B$ is excellent). Let $c$ be an element of $R^{o}$ such that $\left(R_{\mathrm{red}}\right)_{c}$ is regular. Then $c$ has a power which is a completely stable weak test element for $R$. If $R$ is reduced, then $c$ has a power which is a completely stable test element for $R$.

Theorem 6.1 will be deduced from:

(6.2) Theorem. Let $R$ be an algebra of finite type over a complete local ring $(B, m, K)$ of characteristic $p$. Let $c \in R^{o}$ be such that $\left(R_{\mathrm{red}}\right)_{c}$ is Gorenstein and $F$-regular. Then $c$ has a power which is a completely stable $q^{\prime}$-weak test element for $R$. If $R$ is reduced, then $c$ has a power which is a completely stable test element for $R$.

This result is contained in Theorem 6.20 below. The proof of Theorem 6.1 immediately follows the proof of Theorem 6.20.

In $\S 7$ we shall obtain an improvement of Theorem 6.1, Theorem 7.32 (see also Theorem 7.33) in which the hypothesis on $c$ is weakened to the condition that $\left(R_{\text {red }}\right)_{c}$ be $F$-regular and Gorenstein (instead of regular).

The technique of the proof of Theorem 6.2 is to pass to a flat purely inseparable extension ring $S$ of $R$ in such a way that $S^{1 / p}$ is module-finite over $S$ (so that the theory of $\S 5$ applies to $S$ ) while controlling the ramification of primes sufficiently to guarantee that $S_{c}$ is $F$-regular (and Gorenstein: hence, strongly $F$-regular). This will entail a considerable digression concerning the behavior of complete local rings under various operations that enlarge their residue class fields.

We shall also need to prove some results about the behavior of tight closure under flat inseparable extension: This question is treated in (6.15) through (6.18).

(6.3) Discussion. Let $K$ be a fixed coefficient field for an arbitrary complete local ring $(R, m, K)$. Given an overfield $L$ of $K$, we shall write $L_{K}[[R]]$ or, more simply, $L[[R]]$, for the completion of $L \otimes_{K} R$ at the ideal $m\left(L \otimes_{K} R\right)$. ( $L_{K}[[R]]$ does depend on the choice of the coefficient field $K$, so that, strictly speaking, $K$ should be indicated in the notation.) Note that if $R=K[[x]]$ is a formal power series ring, where $x$ denotes $x_{1}, \ldots, x_{n}$, then $L[[R]] \cong L[[x]]$. (The obvious maps $L \otimes_{K} R \rightarrow L[[x]], L[x] \rightarrow L[[R]]$ induce continuous maps with the same targets but with their domains completed.)

Suppose that $R$ is module-finite over a complete local ring $B$ with the same coefficient field $K$. We claim that $L[[R]] \cong R \otimes_{B} L[[B]]$. Since $R$ is modulefinite over $B$, the maximal ideal of $B$ expands to an ideal primary to the maximal ideal $m$ of $R$ and so $R \otimes_{B} L[[B]]$ is complete with respect to the expansion of $m$. This yields a continuous map $L[[R]] \rightarrow R \otimes_{B} L[[B]]$. The obvious $B$-algebra maps $L[[B]] \rightarrow L[[R]]$ and $R \rightarrow L[[R]]$ yield a continuous map $R \otimes_{B} L[[B]] \rightarrow L[[R]]$. The compositions in either order yield a map which is evidently the identity on the dense subring $R \otimes_{K} L \cong R \otimes_{B}\left(B \otimes_{K} L\right)$.

Thus, if $R=K[[x]] / I\left(x=x_{1}, \ldots, x_{n}\right)$, then $L[[R]] \cong L[[x]] / I L[[x]]$. Alternatively, if we think of $R$ as module-finite over $B=K[[x]]$, then $L[[R]] \cong$ $R \otimes_{B} L[[x]]$. In any case, $L[[R]]$ is faithfully flat over $R$, and its maximal idcal is the expansion of the maximal ideal of $R$. 
Notice that for any intermediate field $K \subseteq K^{\prime} \subseteq L$ we have natural inclusions $R \subseteq K^{\prime}[[R]] \subseteq L[[R]]$ and that $L_{K^{\prime}}\left[\left[K_{K}^{\prime}[[R]]\right]\right] \cong L_{K}[[R]]$ in a natural way.

Note that $L_{K}[[R]]$ discussed here coincides with $R \hat{\otimes}_{K} L$ discussed in (7.39): this is somewhat more general than the version of complete tensor product given in [S], Chapitre V, A2.

(6.4) Discussion. We retain the conventions of (6.3). Let $K=K_{0} \subseteq K_{1} \subseteq$ $K_{2} \subseteq \cdots \subseteq K_{e} \subseteq \cdots$ be an infinite sequence of fields whose union is a field $L$. Then we may form the union $\bigcup_{e} K_{e}[[R]]$. Since this ring is a direct limit of Noetherian local rings and faithfully flat maps such that the maximal ideal of the domain of each map expands to the maximal ideal of its target, this union is a Noetherian local ring, faithfully flat over $R$, whose residue field is $L$, and whose completion is $L[[R]]$. (If there exists a Noetherian ring $T$ faithfully flat over (or even a pure extension of) all the rings in a directed union, the directed union is Noetherian: given a strictly increasing sequence $J_{t}$ of finitely generated ideals from the union, we have that $J_{t} T$ stabilizes. But each of $J_{t}, J_{t+1}$ is the expansion of an ideal coming from one of the rings in the union, say $J_{t}=I_{t}^{e}$, $J_{t+1}=I_{t+1}^{e}$, where $I_{t} \subseteq I_{t+1}$. But then $I_{t} T=I_{t+1} T \Rightarrow I_{t}=I_{t+1} \Rightarrow J_{t}=J_{t+1}$.)

(6.5) Discussion. Now suppose that $R$ is a complete local ring with a coefficient field $K$ of characteristic $p$. We shall say that an increasing sequence of subfields $\left\{K_{e}\right\}_{e}$ of the perfect closure $K^{\infty}$ of $K$ is admissible for $K$ if first, $K_{0}=K$, second, for all $e, K_{e} \subseteq K_{e+1} \subseteq K_{e}^{1 / p}$ (so that if $q=p^{e}$ we have $K \subseteq K_{e} \subseteq K^{1 / q}$ for all $e$ ) and third, there is a finite set of elements $\theta_{1}, \ldots, \theta_{N}$ of $K^{1 / p}$ such that for all $e, \theta_{1}, \ldots, \theta_{N}$ spans $K_{e}^{1 / p}$ as a vector space over $K_{e+1}$. For a fixed sequence of this type we shall denote by $K_{\infty}\langle R\rangle$ the ring $\bigcup_{e} K_{e}[[R]]$ discussed in (6.4). Note that the completion of $K_{\infty}\langle R\rangle$ is $\left(\bigcup_{e} K_{e}\right)[[R]]$.

(6.6) Lemma. Let $R$ be a complete local ring of characteristic $p$ with coefficient field $K$ and let $\left\{K_{e}\right\}_{e}$ be an admissible sequence of extension fields for $K$.

(a) If $S$ is a complete local ring module-finite over $R$ with the same coefficient field then $K_{\infty}\langle S\rangle \cong S \otimes_{R} K_{\infty}\langle R\rangle$.

(b) If $R$ is module-finite over (respectively, homomorphic image of) a regular ring $A=K\left[\left[x_{1}, \ldots, x_{n}\right]\right]$, then $S=K_{\infty}\langle R\rangle$ is module-finite over (respectively, a homomorphic image of) the regular ring $K_{\infty}\langle A\rangle$.

(c) $R \rightarrow K_{\infty}\langle R\rangle$ is a flat, purely inseparable map with Gorenstein fibers. Moreover, $K_{\infty}\langle R\rangle$ is Henselian.

(d) If $S=K_{\infty}\langle R\rangle$, then $S$ is a finite module over $S^{p} \subseteq S$ (i.e., the Frobenius map is a finite morphism). If $S$ is reduced, then, equivalently, $S^{1 / p}$ is module-finite over $S$. In particular, $S$ is excellent.

Proof. (a) follows from the fact that for every $e, K_{e}[[S]] \cong S \otimes_{R} K_{e}[[R]]$, and (b) is immediate from (a). We next prove (c). $K_{\infty}\langle R\rangle$ is Henselian because it is a direct limit of Henselian rings and local homomorphisms. It is purely inseparable over $R$ because $\left(K_{e}[[R]]\right)^{q} \subseteq R$ for every $q=p^{e}$. Flatness follows from (a) and the case where $R=K\left[\left[x_{1}, \ldots, x_{n}\right]\right]$, where it is obvious.

It remains to check that the fibers of the map $R \rightarrow S=K_{\infty}\langle R\rangle$ are Gorenstein. If $P$ is a prime of $R$ we have that $S / P S \cong K_{\infty}\langle R / P\rangle$, and so it suffices to check that the generic fiber is Gorenstein when $R$ is a domain. Suppose 
that $R$ is module-finite over a regular subring $A$ with coefficient field $K$ and that the fraction fields of $A$ and $R$ are $\mathcal{F} \subseteq \mathcal{G}$. The generic fiber is therefore $\mathcal{G} \otimes_{\mathcal{F}}(A-\{0\})^{-1} K_{\infty}\langle A\rangle$. Now, $T=(A-\{0\})^{-1} K_{\infty}\langle A\rangle$ is a localization of a regular ring and, therefore, regular, and so it suffices to note that if $T$ is an arbitrary Gorenstein ring containing a field $\mathcal{F}$ and $\mathcal{G}$ is a finite algebraic extension field of $\mathcal{F}$, then $\mathcal{G} \otimes_{\mathcal{F}} T$ is Gorenstein. (Briefly, $\mathcal{G}$ is the result of killing an $R$-sequence in a polynomial ring over $\mathcal{F}$, since a maximal ideal of a polynomial ring over a field is a complete intersection, and so $\mathcal{G} \otimes_{\mathcal{F}} T$ is the result of killing an $R$-sequence in a polynomial ring over $T$. Cf. [WITO].) This completes the proof of (c).

From (b) it follows that to prove (d) it suffices to consider the case where $R=A=K\left[\left[x_{1}, \ldots, x_{n}\right]\right]$. We want to show that $S^{1 / p}$ is module-finite over $S$. Let $\left\{\mu_{j}\right\}$ denote the finite set of monomials in the elements $x_{i}^{1 / p}$ in which each exponent is an integer between 0 and $p-1$. Let $\left\{\theta_{1}, \ldots, \theta_{N}\right\}$ be elements of $K^{1 / p}$ that span $K_{e}^{1 / p}$ over $K_{e+1}$ as in the definition of an admissible sequence of fields. It will suffice to show that the elements $\theta_{h} \mu_{j}$ span $S^{1 / p}$ over $S$. Since $S$ is the union of the rings $K_{e}\left[\left[x_{1}, \ldots, x_{n}\right]\right]$, it will suffice to note that $\left(K_{e}\left[\left[x_{1}, \ldots, x_{n}\right]\right]\right)^{1 / p}=\left(K_{e}^{1 / p}\right)\left[\left[x_{1}^{1 / p}, \ldots, x_{n}^{1 / p}\right]\right]$, which is spanned over $K_{e+1}\left[\left[x_{1}, \ldots, x_{n}\right]\right] \subseteq S$ by the elements $\theta_{h} \mu_{j}$.

It follows at once [Ku2] that $S$ is excellent.

(6.7) Discussion. To make use of this result, we need a large source of admissible sequences for fields $K$. Part (b) of (6.8) below will be our main provider. We first recall that a $p$-base for a field $K$ of characteristic $p$ is a family $\Lambda$ of elements of $K$ such that $K=K^{p}(\Lambda)$ and such that for every finite subset $\Sigma \subseteq \Lambda$ if $\Sigma$ has $s$ elements then $\left[K^{p}(\Sigma): K^{p}\right]=p^{s}$. Every set of generators for $K$ over $K^{p}$ has a subset which is a $p$-base. See [N], $\S 31$. In this situation, the fields $\left\{K^{p}[\lambda]: \lambda \in \Lambda\right\}$ are mutually linearly disjoint extensions of $K^{p}$ in $K$ of degree $p$, and, if for every $\lambda \in \Lambda$ we choose a power $q(\lambda)$ of $p$, then the fields $\left\{K\left[\lambda^{1 / q(\lambda)}\right]: \lambda \in \Lambda\right\}$ are mutually linearly disjoint extensions of $K$ in $K^{\infty}$ of respective degrees $q(\lambda)$. Thus, as a vector space basis for $K^{\infty}$ over $K$ we may take the set of all monomials $\lambda_{1}^{\eta_{1}} \ldots \lambda_{s}^{\eta_{s}}$ in distinct elements $\lambda_{1}, \ldots, \lambda_{s}$ of $\Lambda$ such that each exponent $\eta_{j}$ is a nonnegative fraction, less than 1 , whose denominator is a power of $p$.

(6.8) Lemma. Let $K$ be a field of characteristic $p$.

(a) Let $k$ be a subfield of $K$ such that $K$ is separable over $k$ and $\operatorname{tr} \operatorname{deg}_{k} K$ $=r$ is finite. Let $K_{e}=\left\{K\left[k^{1 / q}\right]\right\}$ where $q=p^{e}$. Then $\left\{K_{e}\right\}_{e}$ is an admissible sequence of fields for $K$. In fact, $N=\left[K^{1 / p}: K\left[k^{1 / p}\right]\right] \leq p^{r}$, and if $\theta_{1}, \ldots, \theta_{N}$ span $K^{1 / p}$ over $K\left[k^{1 / p}\right]$ then they span $\left(K\left[k^{1 / q}\right]\right)^{1 / p}$ over $K\left[k^{1 / q p}\right]$ for all $q$.

(b) Let $\Lambda$ be a p-base for $K$, let $\Gamma$ be a cofinite subset of $\Lambda$ (i.e., $\Lambda-\Gamma$ is finite), and let $K_{e}^{\Gamma}$ be the subfield of $K^{\infty}$ generated over $K$ by the $\left(p^{e}\right)$ th roots of all elements in $\Gamma$. Then $\left\{K_{e}^{\Gamma}\right\}_{e}$ is an admissible sequence of field extensions for $K$.

Proof. (a) We first note that $K^{1 / p}$ is a finite algebraic extension of $K\left[k^{1 / p}\right]$ of degree at most $p^{r}$. To see this, observe that otherwise we could choose $N_{1}>p^{r}$ elements in $K$ whose $p$ th roots are linearly independent over $K\left[k^{1 / p}\right]$. 
This situation is preserved if we replace $K$ by the field generated by these $N_{1}$ elements over $k$. Thus, we may assume that $K$ is finitely generated over $k$ : In this case $K$ is separably generated over $k$ and we may write $K=k(u)[z]$ where $u$ indicates the elements $u_{1}, \ldots, u_{s}$ and $z$ is a single element which is separable algebraic over $k(u)$. Now, when $z$ is separable algebraic over a field $G, G^{1 / p}[z]$ already contains a $p$ th root of $z$. (The field extensions $G^{1 / p} \subseteq G^{1 / p}\left[z^{1 / p}\right]$ and $G \subseteq G[z]$ are isomorphic, and have the same degree, $m$; but $G[z]$ and $G^{1 / p}$ are linearly disjoint over $G$, and so $\left[G^{1 / p}[z]: G^{1 / p}\right]=m$ as well. Since $G^{1 / p}[z] \subseteq G^{1 / p}\left[z^{1 / p}\right]$ and both have degree $m$ over $G^{1 / p}$, they are equal.) It follows that $(k(u)[z])^{1 / p}=(k(u))^{1 / p}[z]=k^{1 / p}\left(u_{1}^{1 / p}, \ldots, u_{s}^{1 / p}\right)[z]$, which has degree at most $p^{s} \leq p^{r}$ over $k^{1 / p}\left(u_{1}, \ldots, u_{s}\right)[z]$. This establishes that $\left[K^{1 / p}: K\left[k^{1 / p}\right]\right] \leq p^{r}$.

Suppose that $\theta_{1}, \ldots, \theta_{N}$ span $K^{1 / p}$ over $K\left[k^{1 / p}\right]$. It is then clear that the $\theta_{i}$ span $\left(K\left[k^{1 / q}\right]\right)^{1 / p}$ over $K\left[k^{1 / p q}\right]$ for all $q=p^{e}$, since $\left(K\left[k^{1 / q}\right]\right)^{1 / p}=$ $K^{1 / p}\left[k^{1 / p q}\right]$, and the admissibility of the specified sequence $K_{e}$ follows.

(b) Let $\sigma_{1}, \ldots, \sigma_{h}$ be the elements of $\Lambda$ not in $\Gamma$. We take $\theta_{1}, \ldots, \theta_{N}$ to be the monomials in the elements $\sigma_{i}^{1 / p}$ in which every exponent is a nonnegative integer $\leq p-1$. The result is now an easy consequence of the last statement in Discussion 6.7.

We shall need the following result from [Mat], (30.E).

(6.9) Lemma. Let $\left\{L_{j}\right\}_{j \in J}$ be an arbitrary nonempty family of subfields of a field $L$ with $\bigcap_{j} L_{j}=K$. Let $A_{j}=L_{j}\left[\left[x_{1}, \ldots, x_{n}\right]\right] \subseteq B=L\left[\left[x_{1}, \ldots, x_{n}\right]\right]$ and let $\mathcal{F}_{j}$ denote the fraction field of $A_{j}$. Then $\bigcap_{j} \mathcal{F}_{j}=\mathcal{G}$, the fraction field of $A=K\left[\left[x_{1}, \ldots, x_{n}\right]\right]$.

Similarly, the intersection of all of the fraction fields of the polynomial rings $L_{j}\left[x_{1}, \ldots, x_{n}\right]$ is the fraction field of $K\left[x_{1}, \ldots, x_{n}\right]$.

Note that the second statement is completely elementary: by induction on $n$, one may reduce to the case of a single variable, where it follows from the fact that when one writes an element in the fraction field of $L[x]$ in lowest terms, with monic denominator, the result is unique, and hence independent of the field to which one thinks of the coefficients as belonging.

We shall also need:

(6.10) Lemma. Let $\mathcal{F}$ be a field, let $\left\{\mathcal{F}_{j}\right\}_{j \in J}$ be a family of subfields, directed by $\supseteq$, with intersection $L$, let $K$ be a subfield of $L$, and let $\Omega$ be a subfield of $\mathcal{F}$ that is a finite algebraic extension of $K$. Suppose that $L$ and $\Omega$ are linearly disjoint in $\mathcal{F}$ over $K$. Then there exists an element $j \in J$ such that $\mathcal{F}_{j}$ (as well as all smaller members of the family) is linearly disjoint from $\Omega$ over $K$.

Proof. Fix a basis $w_{1}, \ldots, w_{N}$ for $\Omega$ over $K$. As we decrease $\mathcal{F}_{j}$ the dimension of the vector space $V_{j}$ spanned by the $w_{i}$ over $\mathcal{F}_{j}$ can only increase, but can never be more than $N$. Therefore, we can choose $\mathcal{F}_{j}$ so that this dimension is maximum. We want to prove that the $w_{i}$ are independent over $\mathcal{F}_{j}$. If not, after renumbering we may suppose that $w_{1}, \ldots, w_{r}$ is a basis for $V_{j}$ with $r<n$. Then $w_{r+1}$ is an $\mathcal{F}_{j}$-linear combination of $w_{1}, \ldots, w_{r}$ in a unique way. At least one of the coefficients occurring is not in $L$. Since the family is directed, we can find a smaller field in the family that excludes this coefficient. 
Over this field, $w_{1}, \ldots, w_{r}, w_{r+1}$ are independent, contradicting our choice of $\mathcal{F}_{j}$.

(6.11) Discussion and notation. Let $K$ be a field of characteristic $p$ and let $\Lambda$ be a $p$-base for $K$. Let $\Gamma$ be a cofinite subset of $\Lambda$. Then we may define an admissible sequence of fields $K_{e}^{\Gamma}=K\left[\lambda^{1 / p^{e}}: \lambda \in \Gamma\right]$ for $K$ as in (6.8b). If $R$ is a complete local ring with $K$ as coefficient field then we may define $R^{\Gamma}=K_{\infty}^{\Gamma}\langle R\rangle=\bigcup_{e} K_{e}^{\Gamma}[[R]]$. Note that the completion of $R^{\Gamma}$ is $K^{\Gamma}[[R]]$, where $K^{\Gamma}=\bigcup_{e} K_{e}^{\Gamma}$.

All of the results of (6.6) apply. Thus, $R^{\Gamma}$ is faithfully flat and purely inseparable over $R$, with Gorenstein fibers. $R^{\Gamma}$ is module-finite over $\left(R^{\Gamma}\right)^{p}$ and $R^{\Gamma}$ is an excellent Henselian ring. When $S$ is module-finite over $R$ with the same coefficient field $K, S^{\Gamma} \cong S \otimes_{R} R^{\Gamma}$. In particular, if $R^{\Gamma}$ is module-finite over a regular ring $A=K\left[\left[x_{1}, \ldots, x_{n}\right]\right]$ then $R^{\Gamma}$ is module-finite over the regular ring $A^{\Gamma}=\bigcup_{e} K_{e}^{\Gamma}\left[\left[x_{1}, \ldots, x_{n}\right]\right]$. Moreover, if $\Gamma^{\prime} \subseteq \Gamma$ then $R^{\Gamma^{\prime}} \rightarrow R^{\Gamma}$ is faithfully flat and purely inseparable. The inseparability is obvious since $R^{\Gamma}$ is purely inseparable even over $R$. The faithful flatness is clear in the case where $R=K\left[\left[x_{1}, \ldots, x_{n}\right]\right]$ is regular and may then be obtained in general by using the fact that $R$ is module-finite over a regular ring and making a base change.

When $S$ is a finitely generated algebra over a complete local ring $R$ with a $p$-base $\Lambda$ for its coefficient field $K$, we shall write $S^{\Gamma}$ for $S \otimes_{R} R^{\Gamma}$ : this ring is faithfully flat and purely inseparable over $S$ for each $\Gamma$ cofinite in $\Lambda$. $S^{\Gamma}$ is finitely generated over $R^{\Gamma}$ and so has the property that $S^{\Gamma}$ is module-finite over $\left(S^{\Gamma}\right)^{p}$ for every $\Gamma$ cofinite in $\Lambda$.

(6.12) Lemma. Let $K$ be a field of characteristic $p$ and let $\Lambda$ be a fixed $p$ base for $K$. let $K^{\Gamma}, A^{\Gamma}$, etc., be defined as in (6.11). Let $\mathbf{x}$ denote the string $x_{1}, \ldots, x_{n}$.

(a) $\bigcap_{\Gamma} K^{\Gamma}=K$ as $\Gamma$ runs through all cofinite subsets of $\Lambda$.

(b) Let $A=K[[\mathbf{x}]]$. The intersection of the fraction fields of the rings $K^{\Gamma}[[\mathbf{x}]]$ as $\Gamma$ runs through all cofinite subsets of $\Lambda$ is the fraction field of $A$.

$\left(\mathrm{b}^{o}\right)$ Let $A=K[\mathbf{x}]$. The intersection of the fraction fields of the rings $K^{\Gamma}[\mathbf{x}]$ is the fraction field of $K[\mathbf{x}]$.

(c) The intersection of the fraction fields of the rings $A^{\Gamma}$ as $\Gamma$ runs through all cofinite subsets of $\Lambda$ is the fraction field of $A$.

Proof. (a) Recall the description of the $K$-basis for $K^{\infty}=K^{\Lambda}$ given in the last sentence of (6.7). Similarly, the set $\mathcal{B}_{\Gamma}$ of monomials $\lambda_{1}^{\eta_{1}} \cdots \lambda_{s}^{\eta_{s}}$ in distinct elements $\lambda_{1}, \ldots, \lambda_{s}$ of $\Gamma$ such that each exponent $\eta_{j}$ is a nonnegative fraction, less than 1 , whose denominator is a power of $p$, form a vector space basis for $K^{\Gamma}$ over $K$. When an element $\rho$ of $K^{\infty}$ not in $K$ is written as a $K$ linear combination of elements in the specified basis $\mathcal{B}_{\Lambda}$ for $K^{\infty}$ at least one monomial other than one must occur. We need only choose $\Gamma$ small enough to exclude this monomial from $\mathcal{B}_{\Gamma}$ to make sure that $\rho \notin K^{\Gamma}$, since $\mathcal{B}_{\Gamma} \subseteq \mathcal{B}_{\Lambda}$.

(b) and $\left(\mathrm{b}^{o}\right)$ are then immediate from (a) and Lemma 6.9.

(c) follows from (b), since $A^{\Gamma} \subseteq K^{\Gamma}\left[\left[x_{1}, \ldots, x_{n}\right]\right]$.

(6.13) Lemma. Let $(B, m, K)$ be a complete local ring with coefficient field $K$ of characteristic $p$, and let $\Lambda$ be a fixed p-base for $K$. Let $B^{\Gamma}$ be as in 
(6.11). For a finitely generated B-algebra $R$, let $R^{\Gamma}=B^{\Gamma} \otimes_{B} R$.

(a) If $R$ is reduced or a domain then there exists $\Gamma_{0}$ cofinite in $\Lambda$ such that $R_{\Gamma}$ is also reduced or a domain for all $\Gamma \subseteq \Gamma_{0}$.

(b) If $P$ is a prime ideal of $R$ then there exists a cofinite set $\Gamma_{0} \subseteq \Lambda$ such that $Q=P R^{\Gamma}$ is prime for all $\Gamma \subseteq \Gamma_{0}$, and whenever $P R^{\Gamma}$ is prime the fiber of $R \rightarrow R^{\Gamma}$ over $P$ is a field.

Proof. (a) First suppose that $R$ is a domain. We represent $B$ as a finite module over a regular ring $A=K\left[\left[x_{1}, \ldots, x_{n}\right]\right] \subseteq R$. Now $R^{\Gamma} \cong R \otimes_{B} B^{\Gamma} \cong R \otimes_{B}$ $\left(B \otimes_{A} A^{\Gamma}\right) \cong R \otimes_{A} A^{\Gamma}$, so that there is no loss of generality in assuming that $B=A=K[[x]]$. Since $R$ is finitely generated as an $A$-algebra its fraction field $\Omega$ is finitely generated as a field over the fraction field $L$ of $A$. Let $\mathcal{F}_{\Gamma}$ denote the fraction field of $A^{\Gamma}$. We want to show that we can choose $\Gamma$ cofinite but sufficiently small that $R \otimes_{A} A^{\Gamma}$ is a domain, which will be the case iff it is reduced, since $A^{\Gamma}$ is purely inseparable over $A$. This ring is contained in $\Omega \otimes_{L} \mathcal{F}_{\Gamma}$, and so it suffices to show that we can choose $\Gamma$ so that $\Omega \otimes_{L} \mathcal{F}_{\Gamma}$ is reduced. It is clear that if one choice of $\Gamma$ succeeds then so do all smaller choices. It is also clear that the problem gets harder as $\Omega$ gets larger. By replacing $\Omega$ by a larger field we may assume without loss of generality that it is obtained from $L$ by first making a purely transcendental extension $L(y)=L\left(y_{1}, \ldots, y_{s}\right)$ followed by a finite purely inseparable extension followed by a finite separable extension. The purely inseparable part will be contained in $L^{\prime}\left(y_{1}^{1 / q}, \ldots, y_{s}^{1 / q}\right)$ for some finite purely inseparable extension $L^{\prime}$ of $L$ and some $q$. The separable part may be ignored. We change notation, writing $y$ for $y^{1 / q}$, and we may thus assume that $\Omega=L^{\prime}(y)$ where $L^{\prime}$ is a finite purely inseparable extension of $L$. It suffices to show that we can choose $\Gamma$ so that $L^{\prime}(y) \otimes_{L} \mathcal{F}_{\Gamma}$ is reduced. This ring is a localization of a polynomial ring over $L^{\prime} \otimes_{L} \mathcal{F}_{\Gamma}$, and so it suffices to show that for a fixed finite purely inseparable extension $L^{\prime}$ of $L$ we can choose $\Gamma$ cofinite in $\Lambda$ so that $L^{\prime} \otimes_{L} \mathcal{F}_{\Gamma}$ is reduced, and this is the case iff $L^{\prime}$ and $\mathcal{F}_{\Gamma}$ are linearly disjoint over $L$ in $L^{\infty}$. Since $L^{\prime}$ and $L$ are linearly disjoint over $L$ and $\bigcap_{\Gamma} \mathcal{F}_{\Gamma}=L$ as $\Gamma$ runs through the cofinite subsets of $\Lambda$ by (6.12c), it follows from Lemma 6.10 that for all sufficiently small choices of the cofinite sets $\Gamma, L^{\prime}$ and $\mathcal{F}_{\Gamma}$ are linearly disjoint over $L$. This establishes (a) when $R$ is a domain. Also note that for any prime ideal $P$ of $R, P R^{\Gamma}$ is prime for all sufficiently small cofinite sets $\Gamma \subseteq \Lambda$, since $R^{\Gamma} / P R^{\Gamma} \cong(R / P)^{\Gamma}$ and we may apply part (a) to $R / P$.

Suppose that $R$ is reduced. For every minimal prime $P_{i}$ of $R$ and for all sufficiently small cofinite sets $\Gamma$ we have that $P_{i} R^{\Gamma}$ is prime, and since finite intersection of ideals commutes with flat base change it follows that $\bigcap_{i} P_{i} R^{\Gamma}=$ $\left(\bigcap_{i} P_{i}\right) R^{\Gamma}=(0)$ in $R^{\Gamma}$, and so $R^{\Gamma}$ is reduced for all sufficiently small cofinite sets $\Gamma$.

To complete the proof of $(\mathrm{b})$ we need only see that when $Q=P R^{\Gamma}$ is prime then the fiber $R_{P}^{\Gamma} / P R_{P}^{\Gamma}$ is a field. But since $R^{\Gamma}$ is purely inseparable over $R$, this ring has only one prime ideal. Since $\left(R^{\Gamma} / P R^{\Gamma}\right)_{P}$ is a domain of dimension 0 , it is a field.

We next note:

(6.14) Lemma. Consider rings $R, R_{\lambda}$, and $S$ that are Noetherian of characteristic $p$. 
(a) Let $\left\{R_{\lambda}\right\}_{\lambda}$ be a direct limit system such that the limit ring $R$ is Noetherian. Suppose that all the direct limit system maps are pure and carry parameters to parameters (both conditions are automatic if the maps are faithfully flat). Then for every ideal $J$ of $R$, if $J$ is generated by an ideal $I \subseteq R_{\mu}$, then $J^{*}=\bigcup_{\lambda \geq \mu}\left(I R_{\lambda}\right)^{*}$. Hence, if each $R_{\lambda}$ is weakly $F$-regular (respectively, $F$-regular) then $R$ is weakly $F$-regular (respectively, F-regular).

(b) Suppose that $R \subseteq S$ is pure and $R^{o} \subseteq S^{o}$. Suppose that $c \in R$ is a $q^{\prime}$-weak test element in $S$. Then $c$ is a $q^{\prime}$-weak test element in $R$. In particular, if $R \rightarrow S$ is faithfully flat and $c \in R$ is a $q^{\prime}$-weak test element in $S$, then $c$ is a $q^{\prime}$-weak test element in $R$.

(c) Suppose that $R \subseteq S$ is faithfully flat and that $c \in R^{o}$ is a completely stable $q^{\prime}$-weak test element for $S$. Then $c$ is a completely stable $q^{\prime}$-weak test element for $R$.

Proof. (a) Note that, given $J$, since it is finitely generated, we can always choose such a $\mu$ and $I \subseteq R_{\mu}$. It is clear that each $\left(I R_{\mu}\right)^{*} \subseteq J^{*}$. Now, given $v \in J^{*}$ we can choose $c \in R^{o}$ such that $c v^{q} \in I^{[q]} R$ for all large $q$. Choose $\lambda$ sufficiently large that $c, v \in R_{\lambda}$. Since $R_{\lambda} \rightarrow R$ is pure, we obtain $c v^{q} \in I^{[q]} R_{\lambda}$ for large $q$ as well. Since $c \in R^{o}$ and $R_{\lambda} \rightarrow R$ is pure, $c$ must be in $R_{\lambda}^{o}$ (every minimal prime of $R_{\lambda}$ lies under a prime of $R$, and hence lies under a minimal prime of $R$ ). This establishes the first statement in (a). If all the $R_{\lambda}$ are weakly $F$-regular, then $J^{*}=\bigcup_{\lambda \geq \mu}\left(I R_{\lambda}\right)^{*}=\bigcup_{\lambda \geq \mu} I R_{\lambda}=I R=J$. Finally, if every $R_{\lambda}$ is $F$-regular, and $W$ is a multiplicative system in $R$, let $W_{\lambda}=W \cap R_{\lambda}$ and note that $W^{-1} R$ is the direct limit of the system $\left\{W_{\lambda}^{-1} R_{\lambda}\right\}_{\lambda}$.

(b) Let $w \in 0_{M}^{*}$. Then $1 \otimes w \in\left(S \otimes_{R} 0\right)^{*}$ in $\left(S \otimes_{R} M\right)$ and so we have that for all $q \geq q^{\prime}, c(1 \otimes w)^{q}=0$ in $F_{S}^{e}\left(S \otimes_{R} M\right)$. But this means that $1 \otimes\left(c w^{q}\right)$ is 0 in $S \otimes_{R} F^{e}(M)$. Since $R \rightarrow S$ is pure, $F^{e}(M) \rightarrow S \otimes_{R} F^{e}(M)$ is injective. Thus, $c w^{q}$ is 0 in $F^{e}(M)$, and so $c$ is a $q^{\prime}$-weak test element.

(c) We must show that the image of $c$ is a $q^{\prime}$-weak test element in $\left(R_{P}\right)^{r}$ for every prime $P$ of $R$. Choose a prime $Q$ of $S$ lying over $P$. The image of $c$ in $\left(S_{Q}\right)$ is a $q^{\prime}$-weak test element in $\left(S_{Q}\right)^{\prime}$, and $\left(S_{Q}\right)$ is faithfully flat over

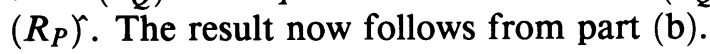

Before proceeding further we need to discuss certain instances in which tight closure behaves well under flat, purely inseparable extension. This digression is comprised of (6.15) through (6.18). The result that we particularly need at present is part (b) of Theorem 6.17.

(6.15) Discussion. If $R \subseteq S$ are rings of characteristic $p$ we say that $S$ is purely inseparable over $R$ if every element in $S$ has a $q$ th power in $R$ for some $q=p^{e}$. In this case, there is a unique prime ideal of $S, \operatorname{Rad} P S$, lying over every prime ideal $P$ of $R$, and every prime ideal $Q$ of $S$ arises in this way from a unique prime ideal of $R$, its contraction to $R$ (since each element of $Q$ has a power in the contraction). This gives a homeomorphism of $\operatorname{Spec} S$ with $\operatorname{Spec} R$.

Given an injective homomorphism $R \rightarrow S$ of Noetherian rings of characteristic $p$ and finitely generated $S$-modules $V \subseteq W$, we say that tight closure 
tests for $V \subseteq W$ can be performed with multipliers in $R$ if for every element $x \in V_{W}^{*}$ there exists an element $c \in R^{o} \cap S^{o}$ such that $c x^{q} \in W^{[q]}$ in $F^{e}(V)$ for all $q \gg 0$. If this is true for all pairs $V \subseteq W$ (with $c \in R^{o} \cap S^{o}$ allowed to depend on $V, W$ and $x \in V$ ) we say that tight closure tests over $S$ can be performed with multipliers in $R$. Of course, this holds whenever there is a ( $q^{\prime}$-weak) test element in $R$ that is also a ( $q^{\prime}$-weak) test element in $S$. What we want to point out here is that when $S$ is purely inseparable over $R$, tight closure tests over $S$ can always be performed with test elements in $R$. The point is that any element $c \in S$ used in the tight closure test will have a $q$ th power in $R$ for some $q=p^{e}$.

We recall from $\S 10$ of [HH4] that if $N \subseteq M$ are finitely generated modules over a Noetherian ring $R$ of characteristic $p$, an element $w \in M$ is said to be in the Frobenius closure of $N$ in $M$ if there exists $q=p^{e}$ such that $w^{q} \in N_{M}^{[q]}$.

(6.16) Theorem. Let $R \rightarrow S$ be a flat homomorphism of Noetherian rings. Let $N \subseteq M$ be finitely generated $R$-modules such that $N_{M}^{*}=N$.

(a) If $S$ is purely inseparable over $R$ and $N^{\left[q^{\prime}\right]}$ is tightly closed in $F^{e^{\prime}}(M)$ either for all $q^{\prime}=p^{e^{\prime}}$ or for some $q^{\prime}$ sufficiently large that $S^{q^{\prime}} \subseteq R$, then the tight closure of $S \otimes_{R} N$ in $S \otimes_{R} M$ is contained in the Frobenius closure. If, moreover, $S$ is $F$-pure, then $S \otimes_{R} N$ is tightly closed in $S \otimes_{R} M$.

(b) If $S$ is purely inseparable over $R, M / N$ is supported only at one maximal $m$ of $R, m S$ is radical ( $\Rightarrow$ maximal) in $S$, and the socle of $M / N$ is one-dimensional, then $S \otimes_{R} N$ is tightly closed in $S \otimes_{R} M$.

(c) If $M / N$ is supported at one maximal ideal $m$ of $R, m S$ is a maximal ideal of $S$, tight closure tests for $N \subseteq M$ can be performed with multipliers in $R$, and the socle of $M / N$ is one-dimensional, then $S \otimes_{R} N$ is tightly closed in $S \otimes_{R} M$.

Proof. In all three parts we may assume that $N=0$ : we replace $M$ by $M / N$.

(a) Suppose that $w \in S \otimes_{R} M$ is in the tight closure of 0 , say $w=\sum_{i=1}^{h} s_{i} \otimes$ $m_{i}$. Then we can choose $q^{\prime}$ such that $s_{i}^{q^{\prime}} \in R$ for every $i$ and such that 0 is tightly closed in $F^{e^{\prime}}(M)$. Thus, $w^{q^{\prime}}$ in $F_{S}^{e^{\prime}}\left(S \otimes_{R} M\right) \cong S \otimes_{R} F^{e^{\prime}}(M)$ may be identified with $1 \otimes \mu$ where $\mu=\sum_{i=1}^{h} s_{i}^{q^{\prime}} m_{i}^{\left[q^{\prime}\right]} \in F^{e^{\prime}}(M)$. Let $c \in S^{o}$ be such that $c w^{q^{\prime} q}=0$ for all $q \gg 0$. Replacing $c$ by a power we may assume that $c \in R$. It follows that $1 \otimes_{R} c \mu^{q}=0$ for all $q \gg 0$, and so $\mu \in 0^{*}$ in $F^{e^{\prime}}(M)$. Thus $\mu=0$ in $F^{e^{\prime}}(M)$, and so $w^{q^{\prime}}=0$ in $F_{S}^{e^{\prime}}\left(S \otimes_{R} M\right)$.

(b) and (c). By virtue of Discussion 6.15, (b) is a special case of (c). Let $w$ denote a socle generator in $M$. Then $1 \otimes w$ generates the socle in $S \otimes_{R} M$ by (7.7c) in the next section, and so it will suffice to show that $1 \otimes w$ is not in the tight closure of 0 in $S \otimes M$. But if $c \in R^{o}$ is such that $c(1 \otimes w)^{q}=0$ in $S \otimes_{R} F^{e}(M)$ for all $e \gg 0$, then, $1 \otimes\left(c w^{q}\right)=0$ in $S \otimes_{R} F^{e}(M)$ for all $e \gg 0$. Since we can consider $M, S \otimes M$ as modules over $R_{m}, S_{m S}$ respectively, and $R_{m} \rightarrow S_{m S}$ is faithfully flat, we obtain $c w^{q}=0$ for all $q \gg 0$ and so $w \in 0^{*}=0$ in $M$, a contradiction.

(6.17) Theorem. Let $R \rightarrow S$ be a flat homomorphism of Noetherian rings of characteristic $p$.

(a) If $R$ is weakly $F$-regular, $R \rightarrow S$ is purely inseparable, $S$ is F-pure, 
and $N \subseteq M$ are finitely generated $R$-modules, then $S \otimes_{R} N$ is tightly closed in $S \otimes_{R} M$

(b) If $R$ is weakly F-regular, $R \rightarrow S$ is purely inseparable, and for every maximal ideal $m \subseteq R, m S$ is radical ( $\Rightarrow$ maximal) in $S$, then $S$ is weakly $F$-regular.

(c) If $R$ is weakly F-regular, every maximal ideal $n \subseteq S$ is of the form $m S$ for a maximal ideal $m$ of $R$, and tight closure tests for $S$-modules can be performed with multipliers in $R$, then $S$ is weakly $F$-regular.

Proof. (a) is immediate from (6.16a), and (b) is a special case of (c). To prove (c) it suffices to show that for every maximal ideal $n$ of $S$ there is a sequence of irreducible $n$-primary ideals $Q_{j}$ cofinal with the powers of $n$ such that $Q_{j}$ is tightly closed in $S$ for each $j$, since every finite length $S$-module will be embeddable in a direct sum of $S / Q_{j}$. But we can choose such a sequence of $m$-primary irreducible ideals in $R$, since $R_{m}$ is normal and, hence, approximately Gorenstein (cf. [Ho4]), and each of these ideals, when expanded to (equivalently, tensored with) $S$ yields an $n$-primary irreducible ideal of $S$ that is tightly closed by virtue of $(6.16 \mathrm{c})$.

Note that in part (c) there is no assumption about the inseparability of $S$ over $R$, but that this raises the issue of whether one can find elements of $R$ that will suffice to perform all tight closure tests in $S$.

(6.18) Example. Let $K$ be a field containing an element $t$ that is not a $p$ th power, let $L=K[\theta]$ where $\theta=t^{1 / p}$, let $R=K\left[\left[x, y, z_{1}, \ldots, z_{n}\right]\right] /(f)$ where $f=x^{p}-t y^{p}-z_{1}^{h_{1}}-\cdots-z_{n}^{h_{n}}$ where every $h_{i}$ is strictly bigger than $p$ and not divisible by $p$ (e.g., we may let all the $h_{i}$ be $p+1$ ), and let $S=L \otimes_{K} R \cong$ $L\left[\left[x, y, z_{1}, \ldots, z_{n}\right]\right] /(f)$. Note that $\left(z_{1}, \ldots, z_{n}\right) R$ is a prime ideal in $R$ and, hence, tightly closed, but that $x-\theta y$ is in the tight closure of $\left(z_{1}, \ldots, z_{n}\right) S$. Thus, tight closure does not commute with flat inseparable base change, even when one is merely enlarging the residue field of a complete local ring in such a way that the maximal ideal does not ramify. Note that $R$ and $R[\theta]$ are hypersurfaces and become regular if we localize at any $z_{i}$ : in particular, both are normal if $n \geq 2$.

We are now ready to prove the following crucial

(6.19) Lemma. Let $(B, m, K)$ be a complete local ring with coefficient field $K$ (respectively, a finitely generated algebra over a field $K$ ), let $R$ be a finitely generated $B$-algebra of characteristic $p$, and let $c \in R^{o}$ be such that $R_{c}$ is weakly $F$-regular and Gorenstein. Let $\Lambda$ be a fixed p-base for the coefficient field $K$ of $B$. For $\Gamma$ cofinite in $\Lambda$ let $R^{\Gamma}=B^{\Gamma} \otimes_{B} R$. Then there exists $a$ choice of $\Gamma_{0}$ cofinite in $\Gamma$ such that for all $\Gamma \subseteq \Gamma_{0}, R_{c}^{\Gamma}$ is weakly $F$-regular and Gorenstein.

Proof. Every nilpotent in $R$ is killed by a power of $c$, since $R_{c}$ is weakly $F$-regular and, hence, reduced. The issues are unaffected by replacing $R$ by $R_{\text {red }}$ by [HH4], $(8.13 \mathrm{~d})$, and we henceforth assume that $R$ is reduced. Let $X=\operatorname{Spec} R_{c}$. Since each $R^{\Gamma}$ is purely inseparable over $R$, each $R_{c}^{\Gamma}$ is purely inseparable over $R_{c}$, and we may identify each $\operatorname{Spec} R_{c}^{\Gamma}$ with $X$. Since the fibers of the flat map $R \rightarrow R^{\Gamma}$ are Gorenstein by $(6.6 \mathrm{c})$ and $R_{c}$ is Gorenstein, 
we have that $R_{c}^{\Gamma}$ is Gorenstein. Since $R_{c}^{\Gamma}$ is a finite module over itself via the Frobenius endomorphism and Gorenstein, the weakly $F$-regular locus is the same as the strongly $F$-regular locus and is open, by (5.5f) and (5.9b). Thus, the set of points in $\operatorname{Spec} R_{c}^{\Gamma}$ where the ring is not weakly ( $\equiv$ strongly) $F$-regular corresponds to a closed set $Z_{\Gamma}$ in $X$. The closed sets in $X$ satisfy DCC, and so we can choose a set $\Gamma_{0}$ with finite complement in $\Lambda$ such that $Z_{\Gamma_{0}}$ is minimal. We shall complete the argument by showing that $Z_{\Gamma}$ must be empty for $\Gamma \subseteq \Gamma_{0}$. Since $Z_{\Gamma}$ decreases with $\Gamma$ (since $R^{\Gamma}$ is faithfully flat over $R^{\Gamma^{\prime}}$ for $\Gamma^{\prime} \subseteq \Gamma$ : see the last paragraph of (6.11)), we may assume that $\Gamma=\Gamma_{0}$.

Assume, to the contrary, that $Z_{\Gamma}$ is not empty, and let $Q$ be a prime ideal of $R$ not containing $c$ corresponding to a point in $Z_{\Gamma}$. It will suffice to show that there is a choice of $\Gamma^{\prime}$ such that $R_{Q}^{\Gamma^{\prime}}$ is weakly $F$-regular, for then $Z_{\Gamma \cap \Gamma^{\prime}}$ will be strictly smaller than $Z_{\Gamma^{\prime}}$ a contradiction. By $(6.17 \mathrm{~b}) R_{Q}^{\Gamma^{\prime}}$ will be weakly $F$ regular provided that $Q R_{Q}^{\Gamma^{\prime}}$ is a prime ( $\Rightarrow$ maximal) ideal, since $R_{Q} \rightarrow R_{Q}^{\Gamma^{\prime}}$ is flat and purely inseparable. But $Q R^{\Gamma^{\prime}}$ is prime for all sufficiently small cofinite $\Gamma^{\prime} \subseteq \Lambda$ by (6.13b).

We are now ready to prove one of our main results:

(6.20) Theorem. Let $(B, m, K)$ be a complete local ring with coefficient field $K$ of characteristic $p$, let $R$ be finitely generated as a B-algebra, and let $c \in R^{o}$ be such that $\left(R_{\mathrm{red}}\right)_{c}$ is weakly $F$-regular and Gorenstein. Then $c$ has a power which is a completely stable $q^{\prime}$-weak test element in $R$. If $R$ is reduced, then $c$ has a power which is a completely stable test element in $R$.

Proof. By [HH4], (8.13d) we may pass to $R_{\text {red }}$, and so it suffices to prove the last statement. Let $\Lambda$ be a $p$-base for $K$ and for each $\Gamma \subseteq \Lambda$ with finite complement let $R^{\Gamma}=B^{\Gamma} \otimes_{B} R$ with $B^{\Gamma}$ as defined in (6.11).

We may then choose $\Gamma$ cofinite in $\Lambda$ so small that $R^{\Gamma}$ is reduced by (6.13a) and such that its localization at the element $c$ is $F$-regular and Gorenstein by (6.19). By (5.10), $c$ has a power which is a completely stable test element in $R^{\Gamma}$ since, by $(6.6 \mathrm{~d})$ and $(6.8 \mathrm{~b}),\left(R^{\Gamma}\right)^{1 / p}$ is module-finite over $R^{\Gamma}$. Since $R^{\Gamma}$ is faithfully flat over $R$, this same power of $c$ is a completely stable test element in $R$, by $(6.14 \mathrm{c})$.

Proof of Theorem 6.1. (a) By [HH4], (8.13d) we may pass to $R_{\text {red }}$. It suffices to show that if $R$ is reduced and $R_{c}$ is regular then $c$ has a power which is a completely stable test element in $\hat{R}$, for it is then automatically a completely stable test element in $R$ as well, by $(6.14 \mathrm{c})$. But since $R \rightarrow \hat{R}$ is flat with regular fibers, so is $R_{c} \rightarrow(\hat{R})_{c}$, and since the base $R_{c}$ is regular so is $(\hat{R})_{c}$. The result is now immediate from (6.20).

The remark about excellent rings now follows from the fact that in an excellent ring the singular locus is open, and in a reduced ring the singular locus is nonempty and its defining ideal has height at least one: thus, one may always choose $c \in R^{o}$ such that $R_{c}$ is regular.

(b) Again, we may assume that $R$ is reduced. Since the fibers of $B \rightarrow \hat{B}$ are smooth, the map $R \rightarrow R \otimes_{B} \hat{B}$ is flat with regular fibers, and since $R_{c}$ is regular it follows that $\left(R \otimes_{B} \hat{B}\right)_{c}$ is regular. We can now apply $(6.20)$. 
We obtain a corollary on the existence of common test elements which will prove very useful in our study of base change in $\S 7$.

(6.21) Theorem. Let $(R, m, K) \rightarrow(S, n, L)$ be a flat local map of local rings such that $\left(R^{o}\right)^{-1} S_{\mathrm{red}}$ is regular, the singular locus in $S_{\mathrm{red}}$ is closed, and such that the map $S \rightarrow \hat{S}$ has regular fibers. Then there is an element $c \in R^{o}$ that is a completely stable $q^{\prime}$-weak test element in all of the rings $R, \hat{R}, S$, and $\hat{S}$. If $S$ is reduced then there is an element $c \in R^{o}$ that is a completely stable test element in all the rings $R, \hat{R}, S$ and $\hat{S}$.

Proof. Let $J$ be the defining ideal of the singular locus in $S_{\text {red }}$. Then the image of $R^{o}$ in $S_{\text {red }}$ must meet $J$, and it follows that we can choose $c \in R^{o}$ such that $\left(S_{\text {red }}\right)_{c}$ is regular. It now follows from (6.1) applied to $S$ that $c$ has a power that is a completely stable $q^{\prime}$-weak test element in $\hat{S}$ (or a test element, if $S$ is reduced), and since $\hat{S}$ is faithfully flat over $\hat{R}, S$, and $R$, the result follows from $(6.14 \mathrm{c})$.

We also state a global version in which we relax the flatness condition considerably:

(6.22) Theorem. Let $R \stackrel{h}{\longrightarrow} S$ be Noetherian rings of characteristic $p$ each of which is finitely generated as an algebra over a local ring such that the maps from these two local rings to their completions have smooth fibers. Suppose that $h\left(R^{o}\right) \subseteq S^{o}$ (this is automatic if $h$ is flat), that the singular loci in $R_{\mathrm{red}}$ and $S_{\mathrm{red}}$ are closed, and that $\left(\left(R^{o}\right)^{-1} S\right)_{\mathrm{red}}$ is regular. Then there exists $c \in R^{o}$ such that $\left(R_{\mathrm{red}}\right)_{c}$ and $\left(S_{\mathrm{red}}\right)_{c}$ are both regular, and so $c$ has a power $c^{\prime}$ such that $c^{\prime}$ is a completely stable weak test element for $R$ and $h\left(c^{\prime}\right)$ is a completely stable weak test element for $S$. If $R$ and $S$ are reduced, then $c$ has a power $c^{\prime}$ which is a completely stable test element in $R$ such that $h\left(c^{\prime}\right)$ is a completely stable test element in $S$.

Proof. As usual, we may assume that both $R$ and $S$ are reduced. We can choose $c \in R^{o}$ such that $R_{c}$ is regular and $d \in R_{o}$ such that $S_{d}$ is regular, and then we replace $c, d$ by $c d$. The result is then immediate from part (b) of Theorem 6.1.

We next prove some results concerning when tight closure is preserved under arbitrary base change. We shall say that an excellent Noetherian ring $R$ of characteristic $p$ has abundant test elements if for every domain $D$ modulefinite over a homomorphic image domain of $R$ every element $c \in D-\{0\}$ such that $D_{c}$ is regular has a power which is a test element. We first note:

(6.23) Proposition. Let $R$ be a Noetherian ring of characteristic $p$. If $R$ is a finitely generated algebra over an excellent local ring or if the Frobenius endomorphism $F: R \rightarrow R$ is a finite morphism, then $R$ has abundant test elements. Proof. Both of the hypotheses imply excellence and pass to module-finite domains over homomorphic images of $R$. The result then follows from (6.1b) in the first case and from (5.10) in the second case.

In fact, both classes of rings discussed in (6.23) satisfy a much stronger condition: in every reduced, finitely generated algebra $S$ over one of these rings, 
every element $c$ such that $S_{c}$ is regular has a power $d$ such that $d$ is in $\tau(T)$ for every ring $T$ which is either a local ring of $S$ or the completion of a local ring of $S$. For these classes, the test elements are more than abundant.

(6.24) Theorem. Let $R \rightarrow S$ be a homomorphism of Noetherian rings of characteristic $p$. Let $N \subseteq M$ be finitely generated $R$-modules and let $w \in M$ be an element of $M$ in $N^{*}$. Assume that at least one of the following conditions holds:

(i) $R$ is excellent and has abundant test elements, or

(ii) $R$ is locally excellent and $S$ has a locally stable test element (or $S$ is local), or

(iii) $S$ has a completely stable test element (or $S$ is a complete local ring).

Then $1 \otimes w$ is in the tight closure of the image of $S \otimes_{R} N$ in $S \otimes_{R} M$.

Proof. In proving this result we may map a finitely generated free module $G$ onto $M$ and replace $N$ by its inverse image in $G$ and $w$ by an element of $G$ mapping onto it. In the remainder of this argument we assume that $M$ is free.

We first prove (i). First note that Proposition (6.25) of [HH4] generalizes to the case of modules. (The strengthened result asserts that $w$ is in the tight closure of $N \subseteq M$ if and only if this holds modulo every minimal prime $P$ of $R$, i.e., the image of $w$ in $M / P M$ is in the tight closure of $\operatorname{Im}(N \rightarrow M / P M)$ for every minimal prime $P$. One can reduce to the case where $M$ is free and the argument given in (6.25) of [HH4] is valid without change.) Thus, if we have a counterexample to the theorem, we obtain one in which $S$ is a domain, for if $1 \otimes w$ is not in the tight closure of $\operatorname{Im}\left(S \otimes_{R} N \rightarrow S \otimes_{R} M\right)$, this will remain true when $S$ is replaced by $S / P$ for a suitable minimal prime $P$. Thus, we may assume that $S$ is a domain. Let $Q=\operatorname{Ker}(R \rightarrow S)$.

Then we may replace $S$ by $R / Q$ as well. For if tight closure is preserved when we apply $(R / Q) \otimes_{R}-$, it will also be preserved when we further apply $S \otimes_{R / Q^{-}}$, for it is always preserved when one tensors with an extension domain of a domain (since nonzero elements map to nonzero elements). Thus, there is no loss of generality in supposing that $S=R / Q$ for a suitable prime $Q$ of $R$. Let $Q=Q_{h} \supseteq Q_{h-1} \supseteq \cdots \supseteq Q_{0}$ be a saturated chain of prime ideals of $R$ descending from $Q$ such that $Q_{0}$ is a minimal prime of $R$. We shall prove by induction on $i$ that tight closure is preserved when we pass from $R$ to $R / Q_{i}$, $0 \leq i \leq h$. For $i=0$ this is clear, for tight closure is always preserved when one kills a minimal prime. To carry through the inductive step, we may replace $R$ by $R / Q_{i-1}$.

We therefore see that, to complete the proof of (i), it suffices to show that if $R$ is a domain and $Q$ is a height one prime ideal of $R$, then tight closure is preserved when we pass to $S=R / Q$. To see this, let $R^{\prime}$ be the integral closure of $R$ in its fraction field (which is module-finite over $R$, since $R$ is excellent), and let $Q^{\prime}$ be a prime ideal of $R^{\prime}$ which lies over $Q$, so that $R / Q \rightarrow R^{\prime} / Q^{\prime}$ is injective and module-finite. Now, tight closure is obviously preserved when we pass from $R$ to $R^{\prime} \supseteq R$. Moreover, since $R^{\prime}$ is excellent normal and $Q^{\prime}$ is height one (so that $R_{Q^{\prime}}^{\prime}$ is regular), there is an element $c \in R^{\prime}-Q^{\prime}$ such that $R_{c}^{\prime}$ is regular. After replacing $c$ by a power we see that we may assume that $c$ is a test element for $R^{\prime}$ not in $Q^{\prime}$. It follows that tight closure will be preserved when we pass from $R^{\prime}$ to $T=R^{\prime} / Q^{\prime}$. Thus, the image of $1 \otimes w$ in $T \otimes_{R} M$ is in the tight closure of $\operatorname{Im}\left(T \otimes_{R} N\right)$. To complete the proof, we must show 
that this implies that the image of $1 \otimes w$ in $S \otimes_{R} M$ is in the tight closure of $S \otimes N$. (Here, $S \subseteq T$ is a module-finite extension of domains.) This follows from Lemma 6.25 given below (we have included a brief proof, although issues of this sort are studied in greater generality in [HH9]).

To handle case (ii), suppose that one has a counterexample. The fact that $S$ has a locally stable test element enables one to preserve the fact that the image of $1 \otimes w$ is not in the tight closure of $\operatorname{Im}\left(S \otimes_{R} N\right)$ while replacing $S$ by a localization at a prime. Thus, one may assume that $S$ is local. But then one may replace $R$ by its localization at the contraction of the maximal ideal of $S$ (and $N, M$ by their localizations). Since $R$ is then excellent local we are in case (i), by (6.23).

Likewise, to prove (iii), we first replace $S$ by a localization and then by the completion of that local ring: the fact that we have a completely stable test element enables us to keep an element out of the tight closure while completing. Thus, we may assume that $S$ is complete local. We may then replace $R, M$, $N$ by their localizations at the prime ideal of $R$ lying under the maximal ideal of $S$, and then by their completions with respect to the maximal ideal of $R$. Once $R$ is complete local we are again in case (i).

To complete the argument we still need:

(6.25) Lemma. Let $S \subseteq T$ be a module-finite extension of Noetherian domains of positive characteristic $p$. Suppose that $N \subseteq M$ are finitely generated $S$ modules with $M$ free such that $w \in M$ has the property that its image in $T \otimes_{S} M$ is in the tight closure of $T N=\operatorname{Im}\left(T \otimes_{S} N \rightarrow T \otimes_{S} M\right)$. Then $w$ is in the tight closure of $N$ in $M$.

Proof. We can choose an $S$-linear map $\phi: T \rightarrow S$ such that $\phi(1)=d \in S-\{0\}$ and we can choose $c \in T-\{0\}$ such that $c(1 \otimes w)^{q} \in(T N)^{[q]} T \otimes M$ for all $q$. We can choose a nonzero multiple of $c$ in $S$, and so we may assume that $c \in S-\{0\}$. It follows that for all $q, c w^{q}$ is a $T$-linear combination of elements of $N_{M}^{[q]} \subseteq T \otimes_{S} M$. Thinking of $M$ as $S^{t}$ and applying the map $\phi$ to each coordinate, we find that $d c w^{q} \in N^{[q]}$ for all $q$, which shows that $w$ is in the tight closure of $N$ in $M$.

(6.26) Remarks. The conclusion of (6.24) is valid if condition (i) is weakened to:

$\left(i^{o}\right)$ For every homomorphic image domain $D$ of $R$ and height one prime $Q$ of $D$, there is a module-finite extension $D^{\prime}$ of $D$, a prime $Q^{\prime}$ of $D^{\prime}$ lying over $Q$, and an element $c \in D^{\prime}-Q^{\prime}$ such that $c$ is a weak test element for $D^{\prime}$.

Moreover, (ii) and (iii) still yield the same conclusion under the weak hypothesis that $S$ have a locally stable weak test element (respectively, a completely stable weak test element). The proofs are essentially unchanged.

In $\S 4$ we observed that a weakly $F$-regular ring is Cohen-Macaulay provided that it is a homomorphic image of a Cohen-Macaulay ring. We conclude this section by recording the observation that the theory of test elements developed here makes the latter hypothesis unnecessary, provided that one assumes instead that the ring is locally excellent. 
(6.27) Proposition. Let $R$ be a locally excellent Noetherian ring of characteristic $p$.

(a) If $R$ is equidimensional, local and the ideal generated by one system of parameters is tightly closed, then $R$ is Cohen-Macaulay and F-rational.

(b) If $R$ is weakly $F$-regular then $R$ is Cohen-Macaulay.

Proof. The hypothesis of weak $F$-regularity passes to the local rings of $R$ at maximal ideals. Thus, (b) follows from (a). Now suppose that $R$ is local, equidimensional, and that the ideal generated by one system of parameters is tightly closed. Since $R$ has a completely stable weak test element ( $R$ is excellent), the system of parameters remains tightly closed when considered in the completion of $R$. Since $R$ is excellent and equidimensional, so is its completion. Since the completion is a homomorphic image of a regular ring, it is Cohen-Macaulay, by Theorem $4.2(\mathrm{c}, \mathrm{d})$. Thus, $R$ itself is Cohen-Macaulay, and so $F$-rational by $(4.2 \mathrm{~d})$.

The following is a global version of (4.3d) that makes use of theory of test elements.

(6.28) Proposition. Let $R$ be a locally excellent Noetherian ring of characteristic $p$ and let $x_{1}, \ldots, x_{d}$ be elements of $R$ such that for every minimal prime ideal $P$ of $R$, the images of $x_{1}, \ldots, x_{d}$ are parameters in $R / P$ (i.e., their images are part of a system of parameters in every local ring of $R / P$ at a prime containing their images). Suppose that $R$ has a locally stable weak test element. Let $J=\left(x_{1}, \ldots, x_{d-1}\right) R$. Then $J^{*}:_{R} x_{d} R=J^{*}$.

Proof. Let $u \in R$ be such that $x_{d} u \in J^{*}$. It suffices to show that $u \in J^{*}$. Let $c$ be a locally stable $q^{\prime}$-weak test element. If $u$ is not in $J^{*}$ then we can choose $q \geq q^{\prime}$ with $c u^{q} \notin J^{[q]}$. This situation will be preserved when we localize at suitable maximal ideal of $R$. In the local ring $S$ so obtained we still have that $u$ is not in $J^{*}$, since the image of $c$ is still a $q^{\prime}$-weak test element. (Notice that the minimal primes of $S$ correspond to a subset of the minimal primes of $R$ under contraction and expansion.)

Thus, we may assume that $R$ is an excellent local domain. The cases where either $J=R$ or $x_{d}$ is a unit are trivial. Thus, we may assume that all of the $x_{i}$ are in the maximal ideal of $R$. Since $R$ is excellent local, it has a completely stable weak test element, and by Proposition (6.1c) of [HH4], it follows that the image of $u$ in the completion $\hat{R}$ of $R$ is not in $(J \hat{R})^{*}$. We thus obtain a counterexample where the ring is complete, local, and equidimensional (since we had reduced to the case where $R$ was an excellent local domain). The result is now immediate from $(4.3 \mathrm{~d})$.

\section{SMOOTH MORPHISMS AND TIGHT CLOSURE}

Our objective in this section is to compare $I^{*} S$ and $(I S)^{*}$ where $R \rightarrow S$ is a smooth morphism of Noetherian rings (or satisfies some modified form of smoothness) of characteristic $p$ and $I \subseteq R$. Here, smooth means flat with geometrically regular fibers: we discuss this notion briefly in (7.5) below. (Note that many authors use the term regular for this notion, and reserve the word smooth for the case where $S$ is finitely presented over $R$.) More generally, we 
want to compare the image of $S \otimes_{R}\left(N_{M}^{*}\right)$ in $S \otimes_{R} M$ with $\left(S \otimes_{R} N\right)_{S \otimes_{R} M}^{*}$ for modules $N \subseteq M$. One hopes that these will be the same, and we can prove this in many instances.

One of the main problems is that we do not know that tight closure commutes with smooth base change even when the base change consists simply of localization. We shall prove that, in good cases, there is a sense in which the problem with localization is the only problem. We should note that tight closure commutes with localization in case $M / N$ has finite length (cf. [HH4], (8.9)) and if $M=R$ and $N=I$ is an ideal generated by a regular sequence: this is the essential content of Theorem 4.5. As indicated in the Introduction, certain other special cases are handled in [AHH]. We do not know any example where tight closure does not commute with localization, but we have not been able to prove that it does in general.

Perhaps the most useful of the results we obtain on base change are summarized in the Theorems 7.1 through 7.4. However, we also want to call attention to Theorem 7.29 and Theorem 7.31, which make explicit some of our general results in important special cases (field extension and adjunction of ordinary and analytic indeterminates), as well as to Theorem 7.42 and Theorem 7.45, which discuss the preservation of tight closure in certain product situations where neither factor is necessarily regular.

(7.1) Theorem. Let $h: R \rightarrow S$ be a smooth morphism of Noetherian rings of characteristic $p$, and suppose that $R$ is locally excellent (or that every local ring of $R$ contains a test element for its completion). Let $N \subseteq M$ be finitely generated $R$-modules.

(a) If $N$ is tightly closed in $M$ and remains so under localization, then $S \otimes_{R} N$ is tightly closed in $S \otimes_{R} M$ and remains so under localization.

(b) If $S$ is projective as an $R$-module and $N$ is tightly closed in $M$ then $S \otimes_{R} N$ is tightly closed in $S \otimes_{R} M$.

Note that throughout this section, when $R \rightarrow S$ is flat and $N \subseteq M$ we tacitly identify $S \otimes_{R} N$ with its image in $S \otimes_{R} M$.

Theorem 7.1 is contained in Theorem 7.18 below.

(7.2) Theorem. If $(R, m, K) \rightarrow(S, n, L)$ is a flat local homomorphism of complete local rings of characteristic $p$ such that the closed fiber $S / \mathrm{mS}$ is regular and $L / K$ is separable, then $\tau(S)=\tau(R) S$, where $\tau$ indicates the ideal generated by the test elements if there is at least one test element (see Definition 3.7 and Proposition 3.8 for details).

Theorem 7.2 is contained in Theorem 7.36.

We also note:

(7.3) Theorem. Let $R \rightarrow S$ be a flat homomorphism of Noetherian rings of characteristic $p$.

(a) Suppose that $\left(R^{o}\right)^{-1} S$ is regular and that $S$ is locally excellent. If for every maximal ideal $m$ of $S$ having contraction $P$ to $R, R_{P}$ is weakly $F$-regular and $S_{m} / P S_{m}$ is regular, then $S$ is weakly $F$-regular. 
(b) Suppose that $R$ is weakly $F$-regular, that $S$ is locally excellent, and that $R \rightarrow S$ has regular fibers over both minimal primes of $R$ and over closed points. Suppose that every maximal ideal of $R$ lies over a maximal ideal of $S$. Then $S$ is weakly $F$-regular.

(c) Suppose that $R$ is $F$-regular, that $S$ is excellent, and that $R \rightarrow S$ has regular fibers. Then $S$ is $F$-regular.

(d) Suppose that $R, S$ are finitely generately algebras over a field $K$. If $R$ is weakly $F$-regular and the fibers over minimal primes and over closed points are regular, then $S$ is weakly $F$-regular.

Theorem 7.3 is contained in Theorem 7.25 below.

(7.4) Theorem. Let $(R, m, K)$ be a local ring and let $N \subseteq M$ be finitely generated $R$-modules. Suppose that $\hat{N}$ is tightly closed in $\hat{M}$. Then for every smooth homomorphism $R \rightarrow S$ such that all maximal ideals of $S$ lie over $m$, $S \otimes_{R} N$ is tightly closed in $S \otimes_{R} M$.

This is Theorem 7.21 below.

The proofs of these results will require a considerable effort.

Note that certain results of this kind in the case where the map $R \rightarrow S$ is flat and purely inseparable are given in $\S 6$ (see Theorems 6.16 and 6.17).

(7.5) Discussion. Various notions of when a homomorphism of Noetherian rings is "smooth" are used in the literature. For our purpose here it will be convenient to define a homomorphism $R \rightarrow S$ of Noetherian rings to be smooth if $S$ is flat over $R$ and for every field $K$ of the form $R_{P} / P R_{P} \cong(R / P)_{(0)}$, $K \otimes_{R} S$ is geometrically regular over $K$, where $T$ is geometrically regular over a field $K$ if for every finite algebraic extension $L$ of $K$ (equivalently, for every finite purely inseparable extension $L$ of $K$ ), $L \otimes_{K} T$ is regular. In particular, in discussing smoothness, we shall not require that $S$ be essentially of finite presentation over $R$, as some authors do. It is easy to see that if $R \rightarrow S$ is smooth and $R$ is regular then $S$ is regular.

If $R \rightarrow S$ is smooth then for every $R$-algebra $\Omega$ essentially of finite type over $R, \Omega \otimes_{R} S$ is again smooth over $\Omega$. For every field $\Omega$ finitely generated over $K=R_{P} / P R_{P}$, and, more generally, for every map $R \rightarrow \Omega$ such that $\Omega$ is essentially of finite type over $R$ and regular, the ring $\Omega \otimes_{R} S$ is regular. In fact, whether $\Omega$ is regular or not, $\Omega \otimes_{R} S$ is smooth over $\Omega$, which forces it to be regular when $\Omega$ is regular. When $R=K$ is a field, $S$ is smooth over $K$ iff $S$ is geometrically regular over $K$.

Smoothness is preserved by composition and is local both on the maximal ideals of $S$ and on the prime ideals of $R$ lying under maximal ideals of $S$.

(7.6) Remark. It is tempting to believe that there should be results corresponding to (7.1), (7.3), and (7.4), for example, when the flat map $R \rightarrow S$ is assumed merely to have geometrically $F$-regular fibers (i.e., that $L \otimes_{R} S$ is $F$-regular for every $L$ which is a finite algebraic extension field of a field $K$ of the form $\left.R_{P} / P R_{P}\right)$ instead of smooth fibers. We have not been able to prove this. Likewise, (7.2) may well be true when $R \rightarrow S$ is a smooth local map of complete local rings, without the hypothesis of separability on the induced map of residue fields. See (7.34).

We begin with a slightly technical but extremely useful result: 
(7.7) Lemma. Let $(R, m, K),\left(R^{\prime}, m^{\prime}, K^{\prime}\right),(S, n, L)$, and $\left(S^{\prime}, n^{\prime}, L^{\prime}\right)$ be arbitrary local Noetherian rings, and suppose that we have a commutative diagram of local homomorphisms

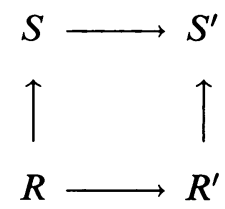

such that $R^{\prime} \rightarrow S^{\prime}$ is flat, $n=m S$, and the images of $L$ and $K^{\prime}$ in $L^{\prime}$ are linearly disjoint over the image of $K$, i.e., such that $L \otimes_{K} K^{\prime} \rightarrow L^{\prime}$ is injective. Suppose that $M$ is an $R^{\prime}$-module, that $V$ is an $R$-module killed by $m$, and that $\theta: V \rightarrow M$ is $R$-linear. Let $\theta^{\prime}: S \otimes_{R} V \rightarrow S^{\prime} \otimes_{R^{\prime}} M$ be the map induced by $\theta$. Then $\operatorname{Ker} \theta^{\prime} \cong S \otimes_{R}(\operatorname{Ker} \theta)$. Note that, since $m$ kills $V$ and $\operatorname{Ker} \theta$, we may identify $S \otimes_{R} V$ with $L \otimes_{K} V$ and $S \otimes_{R}(\operatorname{Ker} \theta)$ with $L \otimes_{K}(\operatorname{Ker} \theta)$.

If $R=K$ and $S=L$ are fields, the result holds even if the condition that the map $R^{\prime} \rightarrow S^{\prime}$ be flat is weakened to the condition that it be pure.

Proof. It will suffice to show that if $\theta$ is injective then $\theta^{\prime}$ is injective, for we may then apply this fact to the induced map $V / \operatorname{Ker} \theta \rightarrow M$. Evidently, $V$ maps into $M_{0}=\operatorname{Ann}_{m} M$. Because $S^{\prime}$ is flat over $R^{\prime}$ we may replace $M$ by $M_{0}$, for $\theta^{\prime}$ may be viewed as the composition of the induced map $L \otimes_{K} V \rightarrow S^{\prime} \otimes_{R^{\prime}} M_{0}$ with the injection $S^{\prime} \otimes_{R^{\prime}} M_{0} \rightarrow S^{\prime} \otimes_{R^{\prime}} M$. Thus, there is no loss of generality in assuming that $m M=0$, and we may consequently replace $R^{\prime} \rightarrow S^{\prime}$ by $R^{\prime} / m R^{\prime} \rightarrow S^{\prime} / m S^{\prime}$ (if we start in the case where $R=K$ and $S=L$ we do not need the flatness of $S^{\prime}$ over $R^{\prime}$ to reduce to this case). We may then replace $R \rightarrow S$ by $K \rightarrow L$ as well. Thus, in the remainder of the proof we shall assume that $R=K$ and $S=L$ are fields. Moreover, having reduced to this case we now weaken the assumption that $R^{\prime} \rightarrow S^{\prime}$ be flat, and, henceforth, we only assume that $R^{\prime} \rightarrow S^{\prime}$ is pure. Note that, by the purity of $R^{\prime} \rightarrow S^{\prime}, M$ embeds into $S^{\prime} \otimes_{R^{\prime}} M$. This implies that the restriction of $\theta^{\prime}$ to $V$ is simply the composition of $\theta$ with the injection $M \rightarrow S^{\prime} \otimes_{R^{\prime}} M$.

We use induction on $N$ to prove that if $v_{1}, \ldots, v_{N} \in M$ are linearly independent over $K$ then the elements $w_{i}=1 \otimes v_{i} \in S^{\prime} \otimes_{R^{\prime}} M$ are linearly independent over $L$. The cases $N=0,1$ are trivial and we assume that $N \geq 2$. We may choose a subset of the $w_{i}$ which is a minimal set of generators, over $R^{\prime}$, for the $R^{\prime}$-module $\sum_{j=1}^{N} R^{\prime} w_{j} \subseteq S^{\prime} \otimes_{R^{\prime}} M$ : by renumbering we may assume that these minimal generators are $w_{1}, \ldots, w_{h}$, and, clearly, we may assume that $h \geq 1$. Then for $N \geq j>h$ (this set may be empty: in that case, summations extended over the values of $j>h$ should be interpreted as having value 0 ) we have

$$
w_{j}=\sum_{i \leq h} r_{j i}^{\prime} w_{i}, \text { where the } r_{j i}^{\prime} \in R^{\prime} .
$$

Now suppose that we have a nontrivial relation $\sum_{j=1}^{N} \lambda_{j} w_{j}=0$, with the $\lambda_{j} \in L$. We shall obtain a contradiction. By substituting the formulas given by $\left({ }^{*}\right)$ for the $w_{j}$ with $j>h$ we obtain $\sum_{i \leq h}\left(\lambda_{i}+\sum_{j>h} r_{j i}^{\prime} \lambda_{j}\right) w_{i}=0$. If the coefficient of $w_{1}$ is not in $n^{\prime}$ then we may invert it, and we discover that $w_{1} \in \sum_{i=2}^{h} S^{\prime} w_{j}$. By the purity hypothesis on $R^{\prime} \rightarrow S^{\prime}$ we see that $w_{1} \in$ $\sum_{i=2}^{h} R^{\prime} w_{j}=H$ (otherwise, the map $M / H \rightarrow S^{\prime} \otimes_{R^{\prime}}(M / H)$ will have $w_{1}+H$ 
in its kernel). But this contradicts the minimality of the generators $w_{1}, \ldots, w_{h}$ for $\sum_{j} R^{\prime} w_{j}$ as an $R^{\prime}$-module.

This shows that $\lambda_{1}+\sum_{j>h} r_{j 1}^{\prime} \lambda_{j} \in n^{\prime}$, so that if $\alpha_{j}^{\prime}$ denotes the image of $r_{j 1}^{\prime}$ in $K^{\prime}$ we have that $\lambda_{1}+\sum_{j>h} \alpha_{j}^{\prime} \lambda_{j}=0$ in $L^{\prime}$. Since $L$ and $K^{\prime}$ are linearly disjoint over $K$ in $L^{\prime}$, there is also an equation $\lambda_{1}+\sum_{j>h} \alpha_{j} \lambda_{j}=$ 0 with $\alpha_{j} \in K$. But then the equation $\sum_{i=1}^{n} \lambda_{i} w_{i}=0$ can be rewritten as $\sum_{i=2}^{h} \lambda_{i} w_{i}+\sum_{i>h} \lambda_{i}\left(w_{i}-\alpha_{i} w_{1}\right)=0$, which contradicts the induction hypothesis on $N$ applied to the $N-1$ vectors

$$
v_{2}, \ldots, v_{h}, v_{h+1}-\alpha_{h+1} v_{1}, \ldots, v_{N}-\alpha_{N} v_{1},
$$

since these are linearly independent over $K$.

(7.8) Corollary. Suppose that $(R, m, K) \rightarrow(S, n, L)$ is a flat local homomorphism of local rings of characteristic $p$ such that $m S=n$ and $L$ is separable over $K$. Let $V$ be an $R$-module killed by $m$ and $M$ an $R$-module. Let $\theta: V \rightarrow M$ be an $F^{e}$-linear map. Then $\operatorname{Ker}\left(F_{S}^{e} \otimes_{R} \theta\right) \cong S \otimes \operatorname{Ker} \theta$.

Proof. Apply Lemma 7.7, letting $R^{\prime}=R$ and $S^{\prime}=S$, letting both vertical maps be the given flat local homomorphism $R \rightarrow S$, while taking the maps $R \rightarrow R^{\prime}$ and $S \rightarrow S^{\prime}$ to be $F_{R}^{e}$ and $F_{S}^{e}$. The linear disjointness condition needed is equivalent to the separability of $L$ over $K$ : if we identify $L^{\prime}$ with $L, K^{\prime}$ is identified with $K, L$ with $L^{q}$ and $K$ with $K^{q}$.

(7.9) Proposition. Let $R$ be a Noetherian ring of characteristic $p$, let $m$ be a maximal ideal, let $M$ be a finitely generated $R$-module and $N$ a submodule such that $M / N$ is supported only at $m$. Let $V$ denote $\operatorname{Ann}_{M / N} m \cong$ $\left(N:_{M} m\right) / N$. Suppose that $c^{\prime} \in R$ and let $W_{q}$ denote the kernel of the map $V \rightarrow F^{e}(M) / N^{[q]} \cong F^{e}(M / N)$ sending the element represented by $w \in N: M m$ to $c^{\prime} w^{q}+N^{[q]}$.

(a) Let $N^{\prime}=\left\{w \in M: c^{\prime} w^{[q]} \in N^{[q]}\right.$ for all $\left.q \geq q^{\prime}\right\}$, so that $N^{\prime}=N^{*}$ if $c^{\prime}$ is a $q^{\prime}$-weak test element. Then $N=N^{\prime}$ if and only if there exist finitely many integers $q(1), \ldots, q(r) \geq q^{\prime}$ such that $W_{q(1)} \cap \cdots \cap W_{q(r)}=0$. More precisely, $\left(N^{\prime} \cap\left(N:_{M} m\right)\right) / N$ may be identified with $\bigcap_{q \geq q^{\prime}} W_{q}$.

(b) Suppose, moreover, that $(R, m, K)$ is local and that $(S, n, L)$ is a flat local $R$-algebra such that $n=m S$ and $L / K$ is separable. Suppose that there exists an element $\omega \in S \otimes_{R} M-S \otimes_{R} N$ such that $c^{\prime} \omega^{q} \in\left(S \otimes_{R} N\right)^{[q]}$ for all $q \geq q^{\prime}$. Then there exists an element $w$ of $M-N$ such that $c^{\prime} w^{q} \in N^{[q]}$ for all $q \geq q^{\prime}$, i.e., such that $w \in N^{\prime}$. Moreover, $w$ may be chosen so that its image in $M / N$ is in $V$.

(c) Suppose that $R$ has a $q^{\prime \prime}$-weak test element $c^{\prime \prime}$ and that $c^{\prime} \in R^{o}$. If $N$ is tightly closed in $M$ then $W_{q}=0$ for all sufficiently large $q$. In fact, if we define $T_{q} \supseteq W_{q}$ to be the set of all elements in $V$ represented by $w \in N: M \quad m$ such that $c^{\prime} w^{q} \in\left(N^{[q]}\right)^{*}$ in $F^{e}(M)$ then the sequence $\left\{T_{q}\right\}$ is decreasing and $T_{q}=0$ for all $q \gg 0$.

Proof. (a) There is no loss of generality in replacing $M, N$ by $M / N$ and 0 , respectively. Thus, we may assume that $M$ has finite length and that $N=0$. If $0^{\prime}$ is strictly larger than 0 it must contain an element which is killed by $m$, since $M$ has finite length. Thus, $0=0^{\prime}$ iff $0=0^{\prime} \cap V$. Now, an element 
$v \in V$ is in $0^{\prime}$ iff $v \in W_{q}$ for all $q \geq q^{\prime}$, by the definition of $0^{\prime}$. Thus, $0^{\prime} \cap V=\cap_{q \geq q^{\prime}} W_{q}$. It follows that $0=0^{\prime}$ iff $\bigcap_{q \geq q^{\prime}} W_{q}=0$. Since $V$ is a finite-dimensional vector space, this intersection is 0 iff some finite subfamily of the $\left\{W_{q}: q \geq q^{\prime}\right\}$ has intersection 0 .

(b) If we assume that there is no such element in $N^{\prime}$ then we can choose powers of $p$, say $q(1), \ldots, q(r)$, all greater than or equal to $q^{\prime}$, such that $\bigcap_{j} W_{q(j)}=0$, which implies that $\bigcap_{j} S \otimes_{R} W_{q(j)}=0$ in $S \otimes_{R} V$. Since $V$ and the $W_{q(j)}$ are killed by $m$ and $m S=n$, we may write $L \otimes_{K} W_{q(j)}$ and $L \otimes_{K} V$ instead. Now, we may identify $L \otimes_{K} V$ with the socle in $S \otimes_{R} M / S \otimes_{R} N \cong$ $S \otimes_{R}(M / N)$ (again using that $m S=n$ and that $S$ is $R$-flat), and (7.8) allows us to identify $L \otimes_{K} W_{q}$ with the kernel $W_{q}^{\prime}$ of the map from $L \otimes_{K} V$ to $F_{S}^{e}\left(S \otimes_{R} M\right) /\left(S \otimes_{R} N\right)^{[q]}$ which sends the class of $\omega$ to the class of $c^{\prime} \omega^{q}$. Since $\cap W_{q(i)}^{\prime}=0$, the desired conclusion follows from part (a) applied over $S$.

(c) As in part (a) we may assume that $N=0$ and that $M$ is supported only at $m$. It is clear that $T_{q} \supseteq W_{q}$. To see that $T_{p q} \subseteq T_{q}$ suppose that $c^{\prime} v^{p q} \in 0^{*}$ in $F^{e+1}(M)$. Then $c^{\prime \prime}\left(c^{\prime} v^{p q}\right)^{p^{h}}=c^{\prime \prime} c^{\prime p^{h}} v^{q p^{h+1}}=0$ in $F^{e+1+h}(M)$ for all $p^{h} \geq q^{\prime \prime}$, and this implies that $c^{\prime \prime}\left(c^{\prime} v^{q}\right)^{p^{h+1}}=0$ in $F^{e+(h+1)}(M)$ for all $p^{h} \geq q^{\prime \prime}$, since the exponent on $c^{\prime}$ in the latter expression has increased. Thus, $c^{\prime} v^{q} \in 0^{*}$ in $F^{e}(M)$, as we wanted. Since the $T_{q}$ are decreasing, to complete the argument it will suffice to show that if $v \in \bigcap_{q} T_{q}$ then $v \in 0^{*}$. But if $v \in \bigcap_{q} T_{q}$ then for all $q, c^{\prime} v^{q} \in 0^{*}$ in $F^{e}(M)$, and so $c^{\prime \prime}\left(c^{\prime} v^{q}\right)^{q^{\prime \prime}}=\left(c^{\prime \prime} c^{\prime q^{\prime \prime}}\right) v^{q q^{\prime \prime}}=0$ in $F^{e+e^{\prime \prime}}(M)$ for all $q=p^{e}$. Thus, $v \in 0^{*}$ in $M$.

Theorem 7.12 below contains a central part of the argument for proving that tight closure behaves well under smooth base change in the sense indicated in Theorem 7.1. Before giving this result, we need a lemma recalling some basic facts, and a discussion of the existence of common test elements.

(7.10) Lemma. Let $(R, m, K) \rightarrow(S, n, L)$ be an arbitrary flat local homomorphism.

(a) If $z \in n$ is not a zerodivisor on $S / m S$, then $z$ is not a zerodivisor on $S$ and $R \rightarrow S / z S$ is again a (faithfully) flat local homomorphism.

(b) More generally, if $z_{1}, \ldots, z_{d} \in n$ form a regular sequence on $S / m S$, then they form a regular sequence in $S$ and $R \rightarrow S /\left(z_{1}, \ldots, z_{d}\right) S$ is again a faithfully flat local homomorphism. The elements $z_{1}, \ldots, z_{d}$ also form an $S$-sequence on $S \otimes_{R} M$ for every nonzero finitely generated $R$-module $M$.

(c) Suppose that $M$ has finite length over $R$ with $V=\operatorname{Ann}_{M} m$, and that $S / m S$ is zero-dimensional with socle $Q$. Then we have an injection $Q \otimes_{K} V \rightarrow S / m S \otimes_{K} V \cong S \otimes_{R} V \rightarrow S \otimes_{R} M$, under which $Q \otimes_{K} V$ is sent onto the socle in $S \otimes_{R} M$.

(d) If $S / m S$ is Gorenstein, $z_{1}, \ldots, z_{d} \in n$ are elements whose images in $S / m S$ are a system of parameters, $I_{t}=\left(z_{1}^{t}, \ldots, z_{d}^{t}\right) S$, and $E_{R}(K)$, $E_{S}(L)$ denote injective hulls for $K=R / m$ over $R$ and $L=S / n$ over $S$, respectively, then $E_{S}(L) \cong\left(\lim _{t} S / I_{t}\right) \otimes_{R} E_{R}(K)$ where the map $S / I_{t} \rightarrow$ $S / I_{t+1}$ is induced by multiplication by $z_{1} \ldots z_{d}$. In other words, if $I=$ $\left(z_{1}, \ldots, z_{d}\right) S$ then $E_{S}(L) \cong H_{I}^{d}(S) \otimes_{R} E_{R}(K)$. Moreover, for every module $W$ of finite length over $S$ which is an essential extension of $L$, 
there exists a module $M$ of finite length over $R$ which is an essential extension of $K$ and an integer $t$ such that $W$ can be embedded in $S / I_{t} \otimes_{R} M$.

Proof. (a) is a special case of Corollary (20.F) on p. 151 of [Mat] and the first statement in (b) is immediate from (a) by a straightforward induction. The second statement reduces to the case where $M=R / I$ is a cyclic module, since $M$ has a filtration by cyclic modules: but $S / I S$ is flat over $R / I$, and we may then apply the first statement of (b).

For the last sentence of (c), note that the first of the three maps is injective since $K$ is a field, and the third is injective because $S$ is $R$-flat. The elements of the socle of $S \otimes_{R} M$ are killed by $m$, and so lie in $\operatorname{Ann}_{m}\left(S \otimes_{R} M\right)$, which, since $S$ is flat, may be identified with $S \otimes_{R} \mathrm{Ann}_{m} M=S \otimes_{R} V \cong(S / m S) \otimes_{K} V$, and it is then clear that $\operatorname{Hom}_{S}\left(L,(S / m S) \otimes_{K} V\right) \cong \operatorname{Hom}_{S / m S}(L, S / m S) \otimes_{K} V \cong$ $Q \otimes_{K} V$.

It remains to establish (d). Since $E_{K}(R)=\bigcup_{h} E_{R / m^{h}}(K)$ when $E_{R / m^{h}}(K)$ is identified with $\operatorname{Ann}_{E_{R}(K)} m^{h}$ and, similarly, $E_{S}(L)=\bigcup_{h} E_{S / m^{h} S}(L)$, we can reduce to the case where $R$ is an Artin local ring. Suppose we write $E=E_{R}(K)$. It will suffice to show that for every $t, E \otimes_{R} S / I_{t}$ is the injective hull of $L$ over $S / I_{t}: S / I_{t}$ is still flat over $R$. Thus, we may assume that $S$ is zero-dimensional and flat over $R$, with a Gorenstein closed fiber. Since $E$ and $R$ both have filtrations by $\ell(R)$ copies of $K$, the length of both $E \otimes_{R} S$ and $R \otimes_{R} S \cong S$ is $\ell(R) \ell(S / m S)$. By part (c), $E \otimes S$ has a one-dimensional socle, and since it has the same length as $S$, it must be an injective hull for $L$ over $S$.

The final statement is then immediate from the fact that both $E_{R}(K)$ and $E_{S}(L)$ are the directed union of the finite length essential extensions of $K$ over $R$ and $L$ over $S$, respectively.

(7.11) Discussion. In the results that follow we frequently need to know for an extension $R \rightarrow S$ that there is an element of $R$ that serves as a test element in both rings. We recall from Theorem 6.21 that if $R \rightarrow S$ is a flat map of local rings such that $\left(R^{o}\right)^{-1} S_{\text {red }}$ is regular, the singular locus in $S_{\text {red }}$ is closed, and $S \rightarrow \hat{S}$ is regular, then there is an element $c \in R^{o}$ that is a completely stable $q^{\prime}$-weak test element in all of the rings $R, \hat{R}, S$, and $\hat{S}$. If $S$ is reduced, $c$ can be chosen to be a completely stable test element in all four rings.

We note that in the special case where $(R, m, K) \rightarrow(S, n, L)$ is a flat local homomorphism of complete local rings such that the closed fiber, $S / m S$, is regular and $L / K$ is separable, there is a much simpler way to see that there is an element $c \in R$ which is a $q^{\prime}$-weak test element for both $R$ and $S$. By [HH4], (8.13d) we may pass to the case where $R$ is reduced (the ideal of nilpotents in $R$ expands to the ideal of nilpotents in $S$ ).

Choose a copy of $K \subseteq R$ to serve as coefficient field and represent $R$ as module-finite over $A=K\left[\left[x_{1}, \ldots, x_{n}\right]\right]$. Since $L$ is separable over $K$, we may extend $K$ to a coefficient field $L \subseteq S$. Let $y_{1}, \ldots, y_{d}$ be elements of $n$ whose images in $S / \mathrm{mS}$ form a regular system of parameters. Then $A \subseteq B=$ $L\left[\left[x_{1}, \ldots, x_{n}, y_{1}, \ldots, y_{d}\right]\right] \subseteq S$ and let $T=B \otimes_{A} R$, which has an obvious map $T \rightarrow S$. Since $T, S$ are complete and flat over their common subring $R$, which is fixed by the map, and since the induced map of closed fibers is an isomorphism, $T \cong S$. (The map is onto, since it is surjective on the residue 
fields, it is clear that the maximal ideal of $T$ maps onto that of $S$, and the rings are complete. To get injectivity, note that it suffices to prove this modulo powers of the maximal ideal of $R$. Thus, we may assume that $R$ is Artin, and, by Noetherian induction, that the kernel is contained in the ideal generated by the socle of $R$ in $T$. But the kernel is $R$-flat and killed by the maximal ideal of $R$. It follows that the kernel is 0 or else $R=K$, and we are done in either case.) But the result is then immediate from [HH4], (6.17): the weak test element for $R$ constructed there retains that property when we tensor with a locally excellent regular $A$-algebra.

Theorem 7.12 is a central tool in our theory of how tight closure is affected by regular base change. The first four parts, which are listed with lowercase Roman numerals, describe behavior without the assumption that the induced extension of residue fields $L / K$ is separable. The last three parts, listed as (a), (b), and (c), give stronger results which hold when $L / K$ is separable.

Before giving the statement we recall from $\S 10$ of [HH4] the notion of tight closure with respect to a family of ideals $\mathcal{C}$ directed by $\supseteq$. Given a Noetherian ring $R$ of characteristic $p$ and a submodule $N$ of a finitely generated $R$ module $M$, we say that $u$ is in the tight closure of $N$ with respect to $\mathcal{C}$ if there exists an ideal $C \in \mathcal{C}$ such that $C x^{q} \in N^{[q]}$ in $F^{e}(M)$ for all $q \gg 0$. The usual notion corresponds to tight closure with respect to the family of ideals generated by an element of $R^{o}$. The notion is used for the case where $\mathcal{C}$ consists of a single principle ideal in part (iii) of Theorem (7.12).

(7.12) Theorem. Let $(R, m, K) \rightarrow(S, n, L)$ be a flat local homomorphism such that $S / m S$ is regular. Let $N \subseteq M$ be finitely generated $R$-modules such that $M / N$ has finite length. Suppose also that there is an element $c \in R^{o}$ that is a $q^{\prime}$-weak test element for both $R$ and $S$, which, by (7.8) above, is always the case if, for example, $R$ and $S$ are complete and $L / K$ is separable. Let $z_{1}, \ldots, z_{d} \in n$ be elements whose images in $S / m S$ are a regular system of parameters. Then:

(i) If $S \otimes_{R} N+\left(z_{1}, \ldots, z_{d}\right)\left(S \otimes_{R} M\right)$ is tightly closed in $S \otimes_{R} M$, then $S \otimes_{R} N+I\left(S \otimes_{R} M\right)$ is tightly closed in $S \otimes_{R} M$ for every ideal I generated by monomials in the elements $z_{1}, \ldots, z_{d}$. In particular, $S \otimes_{R} N$ is tightly closed in $S \otimes_{R} M$.

(ii) If $S \otimes_{R} N+\left(z_{1}, \ldots, z_{d}\right)\left(S \otimes_{R} M\right)$ is not tightly closed in $S \otimes_{R} M$, then there is an element of the tight closure whose image in $S \otimes_{R}(M / N)$ may be identified with an element of $L \otimes_{K} V$, where $V$ is the socle in $M / N$.

(iii) Let $\bar{S}=S /\left(z_{1}, \ldots, z_{d}\right)$. If $\bar{S} \otimes_{R} N$ is tightly closed with respect to $\{c \bar{S}\}$ in $\bar{S} \otimes_{R} M$ then $S \otimes_{R} N+I\left(S \otimes_{R} M\right)$ is tightly closed in $S \otimes_{R} M$ for all ideals $I$ generated by monomials in the elements $z_{1}, \ldots, z_{d}$.

(iv) If $N$ is tightly closed in $M$ and the socle of $M / N$ is one-dimensional, then $S \otimes_{R} N+I\left(S \otimes_{R} M\right)$ is tightly closed in $S \otimes_{R} M$ for every ideal I generated by monomials in $z_{1}, \ldots, z_{d}$.

Moreover, if $L / K$ is separable then:

(a) $S \otimes_{R}\left(N_{M}^{*}\right)=\left(S \otimes_{R} N\right)_{S \otimes_{R} M}^{*}$.

(b) If $N$ is tightly closed in $M$ then $S \otimes_{R} N+I\left(S \otimes_{R} M\right)$ is tightly closed in $S \otimes_{R} M$ for every ideal I generated by monomials in $z_{1}, \ldots, z_{d}$.

(c) If $N$ is tightly closed in $M$ then $S \otimes_{R} N$ is tightly closed in $S \otimes_{R} M$. 
Proof. We may replace $M, N$ by $M / N$ and 0 without affecting any relevant issues. Henceforth we assume that $N=0$ and that $M$ has finite length. Let $V$ denote the socle in $M=M / N$.

We first establish (i) and (ii). Let $I_{t}$ denote the ideal $I+\left(z_{1}^{t}, \ldots, z_{d}^{t}\right)$. If we know that $I_{t}\left(S \otimes_{R} M\right)$ is tightly closed for all $t$ then the result for $I$ follows, since that will place the tight closure of $I\left(S \otimes_{R} M\right)$ in the $n$-adic closure of $I\left(S \otimes_{R} M\right)$. Thus, there is no loss of generality in assuming that $I$ contains a power of each $z_{i}$. It is easy to see that such an $I$ is the intersection of two larger such $I$ 's, unless it has the form $I=\left(z_{1}^{a_{1}}, \ldots, z_{d}^{a_{d}}\right)$ for nonnegative integers $a_{j}$ (cf. [HoE]). But if $I=I^{\prime} \cap I^{\prime \prime}$, then $I\left(S \otimes_{R} M\right)=I^{\prime}\left(S \otimes_{R} M\right) \cap I^{\prime \prime}\left(S \otimes_{R} M\right)$ : this follows from the fact that, by (7.10b), the $z$ 's form a regular sequence on $S \otimes_{R} M$. Thus, if $I^{\prime}\left(S \otimes_{R} M\right)$ and $I^{\prime \prime}\left(S \otimes_{R} M\right)$ are tightly closed, so is $I\left(S \otimes_{R} M\right)$. We have therefore reduced to studying the case where $I=$ $\left(z_{1}^{a_{1}}, \ldots, z_{d}^{a_{d}}\right)$. There is nothing to prove if any $a_{i}$ is 0 . We henceforth assume that the $a_{i}$ are positive integers.

By $(7.10 \mathrm{c})$, the socle in $(S / I) \otimes_{R} M \cong\left(S \otimes_{R} M\right) / I\left(S \otimes_{R} M\right)$ is $(L z) \otimes_{R} V \cong$ $\left(L \otimes_{K} V\right) z$ where $z=\prod_{j} z_{j}^{a_{j}-1}$ and $V$ is the socle in $M$. If 0 is not tightly closed in $(S / I) \otimes_{R} M$ then some element of the socle must be in the tight closure of 0 . Write this element as $\omega z$ where $\omega \in L \otimes_{K} V$. Then for all $q \geq q^{\prime}$ we have that $c \omega^{q} z^{q}$ is 0 in $F_{S}^{e}\left((S / I) \otimes_{R} M\right) \cong\left(S / I^{[q]}\right) \otimes_{R} F^{e}(M) \cong$ $H / I^{[q]} H$, where $H=S \otimes_{R} F^{e}(M) \cong F_{S}^{e}\left(S \otimes_{R} M\right)$. By (7.10b), the $z_{j}$ form a regular sequence on $H$, and it follows that $\left(I^{[q]} H\right):_{H} z^{q} S=\left(z_{1}^{q}, \ldots, z_{d}^{q}\right) H$. Thus, $c \omega^{q} \in\left(z_{1}^{q}, \ldots, z_{d}^{q}\right) F_{S}^{e}\left(S \otimes_{R} M\right)$ for all $q \geq q^{\prime}$, and hence the element $\omega \in L \otimes_{K} V \subseteq S \otimes_{R} M$ is in the tight closure of $\left(z_{1}, \ldots, z_{d}\right)\left(S \otimes_{R} M\right)$. This establishes both part (i) and part (ii).

To prove (iv) it suffices to show that $\left(z_{1}, \ldots, z_{d}\right)\left(S \otimes_{R} M\right)$ is tightly closed in $S \otimes_{R} M$, and, by part (ii), if not there is an element of $L \otimes_{K} V$ in the tight closure. Since $V$ is one-dimensional this element has a nonzero multiple $v$ in $V$, and so we find that $c v^{q}=0$ in $\left(S /\left(z_{1}^{q}, \ldots, z_{d}^{q}\right) S\right) \otimes_{R} F^{e}(M)$ for every $q \geq q^{\prime}$. Since $v \in M$ and $S /\left(z_{1}^{q}, \ldots, z_{d}^{q}\right) S$ is faithfully flat over $R$ by (7.10a), we have that $c v^{q}=0$ in $F^{e}(M)$ for every $q \geq q^{\prime}$, contradicting the fact that 0 is tightly closed in $M$.

To prove (iii) note that if $\omega \in L \otimes_{K} V \subseteq S \otimes_{R} M$ is in the tight closure of the submodule $\left(z_{1}, \ldots, z_{d}\right)\left(S \otimes_{R} M\right)$ then for all $q \geq q^{\prime}$ we have that $c \omega^{q} \in$ $\left(z_{1}^{q}, \ldots, z_{d}^{q}\right) F_{S}^{e}\left(S \otimes_{R} M\right)$. Note that $R \rightarrow \bar{S}$ is faithfully flat local. Let ${ }^{-}$denote images after tensoring with $\bar{S}$ over $R$. Then $c \bar{\omega}^{q}=0$ in $\bar{S} \otimes_{R} F^{e}\left(S \otimes_{R} M\right)$, which is the same as $F^{e}\left(\bar{S} \otimes_{R} M\right)$ calculated over $\bar{S}$. This shows that $\bar{\omega}$ is in the tight closure of 0 in $\bar{S} \otimes_{R} M$ with respect to $c \bar{S}$, and so must be 0 . But the map $S \otimes_{R} M \rightarrow \bar{S} \otimes_{R} M$ is injective when restricted to $S \otimes_{R} V \cong L \otimes_{K} V$, since, by (7.10c), the socle in $\bar{S} \otimes_{R} M$ may also be identified with $L \otimes_{K} V$. This completes the proof of part (iii).

In the remainder of the argument we assume that $L / K$ is separable. We must establish (a), (b), and (c). First note that $(b) \Rightarrow(c)$, since we may take $I$ to be $(0)$. Moreover, (c) $\Rightarrow$ (a) is clear.

Thus, it suffices to check that (b) holds, and by part (iii) of the theorem it suffices to show that a nonzero element of $L \otimes_{R} V$ cannot be in the tight closure of 0 in $\bar{S} \otimes_{R} M$ with respect to $c \bar{S}$. But $R \rightarrow \bar{S}$ is faithfully flat local, $m \bar{S}$ 
is the maximal ideal in $\bar{S}$, and the extension of residue fields is separable. If there were such an element we could apply $(7.9 \mathrm{~b})$ to conclude that there exists a nonzero element $v \in V$ such that $c v^{q}=0$ for all $q \geq q^{\prime}$, which contradicts the fact that 0 is tightly closed in $M$.

(7.13) Remark. Note that $(R, m, K) \rightarrow(S, n, L)$ may be smooth even when $L$ is not separable over $K$ : this happens even when $R=K$ is a field. For example, let $k$ be a field of characteristic $p$, let $B=k[t, x]$ be the polynomial ring in two variables over $k$ and let $A=k\left[t^{p}+x\right]$. Note that $B=A[t]$ is a polynomial ring in one variable over $A$, and that $A \rightarrow B$ is therefore smooth. Let $S$ be the localization of $B$ at $x B$ and let $R=K$ be the fraction field of $A$, which is a subring of $S$, since $x B \cap A=(0)$. Then $K=k\left(t^{p}+x\right) \subseteq k[t, x]_{(x)}$ is smooth, but the map of $K \rightarrow L \cong k(t)$ sends the field generator $t^{p}+x$ to $t^{p}$ and, hence, is not separable.

Theorem 7.15 gives a sharp version of $(7.12 \mathrm{~b})$ when $R$ is complete: we no longer need to assume that $M / N$ has finite length. We first need:

(7.14) Lemma. Let $(R, m, K)$ be a local ring of characteristic $p$ and let $N \subseteq$ $M$ be finitely generated $R$-modules.

(a) If $R$ has a weak test element then $N^{*}=\bigcap_{t}\left(N+m^{t} M\right)^{*}$ in $M$.

(b) Let $R$ be complete, let $R \rightarrow S$ be flat local, let $I \subseteq S$ be an ideal, and suppose that for every finite length $R$-module $H$ such that $0_{H}^{*}=0$, $I\left(S \otimes_{R} H\right)$ is tightly closed in $\left(S \otimes_{R} H\right)$. Then for every finitely generated $R$-module $M$ and submodule $N$ which is tightly closed in $M$, we have that $S \otimes_{R} N+I\left(S \otimes_{R} M\right)$ is tightly closed in $S \otimes_{R} M$.

Proof. (a) Let $N_{t}$ denote the tight closure of $N+m^{t} M$ in $M$. We know from [HH4], (8.13b) that $N^{*}$ is an intersection of $m$-coprimary tightly closed submodules $N_{\lambda}$ of $M$. If $x \in M-N$ and we choose such an $N_{\lambda}$ with $x \notin N_{\lambda}$ then $N_{\lambda} \supseteq N+m^{t} M$ for some $t$ and so $N_{\lambda} \supseteq N_{t}$ for some $t$. Thus, $N^{*}=\bigcap_{t} N_{t}$.

(b) We apply part (a) to $N=N^{*}$ and so obtain that $N=\bigcap_{t} N_{t}$. Since $M$ is complete, by Chevalley's theorem for every $h$ we may choose $t(h)$ such that $N_{t(h)} \subseteq N+m^{h} M$. Since $M / N_{t(h)}$ has finite length, $S \otimes_{R} N_{t(h)}+I\left(S \otimes_{R} M\right)$ is tightly closed in $S \otimes_{R} M$, and so the tight closure of $S \otimes_{R} N+I\left(S \otimes_{R} M\right)$ in $S \otimes_{R} M$ is contained in $S \otimes_{R} N_{t(h)}+I\left(S \otimes_{R} M\right) \subseteq S \otimes_{R}\left(N+m^{h} M\right)+I\left(S \otimes_{R} M\right)=$ $S \otimes_{R} N+I\left(S \otimes_{R} M\right)+m^{h}\left(S \otimes_{R} M\right)$ for all $h$. Since every submodule of $S \otimes_{R} M$ is $n$-adically closed and $m \subseteq n$, we see that $S \otimes_{R} N+I\left(S \otimes_{R} M\right)$ is tightly closed.

(7.15) Theorem. Let $(R, m, K) \rightarrow(S, n, L)$ be a flat local homomorphism such that $R$ is complete, the closed fiber $S / m S$ is regular and $L / K$ is separable. Let $N \subseteq M$ be arbitrary finitely generated $R$-modules. Then:

(a) $S \otimes_{R}\left(N_{M}^{*}\right)=\left(S \otimes_{R} N\right)_{S \otimes_{R} M}^{*}$.

(b) If $N$ is tightly closed in $M$ and $z_{1}, \ldots, z_{d}$ are elements of $S$ that generate the maximal ideal of the fiber $S / m S$, then $S \otimes_{R} N+I\left(S \otimes_{R} M\right)$ is tightly closed in $S \otimes_{R} M$ for every ideal I generated by monomials in $z_{1}, \ldots, z_{d}$.

(c) If $N$ is tightly closed in $M$ then $S \otimes_{R} N$ is tightly closed in $S \otimes_{R} M$. 
Proof. (b) $\Rightarrow$ (c) by taking $I=(0)$ and (c) $\Rightarrow$ (a) is clear. We need only prove (b). But (b) is immediate from $(7.12 b)$ and $(7.14 b)$.

We next extend (7.12abc) in a different direction.

(7.16) Theorem. Let $(R, m, K) \rightarrow(S, n, L)$ be a flat local homomorphism such that the closed fiber $S / m S$ is regular and $L / K$ is separable. Let $N \subseteq M$ be finitely generated $R$-modules such that $M / N$ has finite length. Suppose also that there is a $c \in R^{o}$ which is a $q^{\prime}$-weak test element for both $R$ and $\hat{R}$ (which is always the case if $R$ is excellent). Then:

(a) $S \otimes_{R}\left(N_{M}^{*}\right)=\left(S \otimes_{R} N\right)_{S \otimes_{R} M}^{*}$.

(b) If $N$ is tightly closed in $M$ and $z_{1}, \ldots, z_{d}$ are elements of $S$ that generate the maximal ideal of the fiber $S / m S$, then $S \otimes_{R} N+I\left(S \otimes_{R} M\right)$ is tightly closed in $S \otimes_{R} M$ for every ideal I generated by monomials in $z_{1}, \ldots, z_{d}$.

(c) If $N$ is tightly closed in $M$ then $S \otimes_{R} N$ is tightly closed in $S \otimes_{R} M$.

Proof. (b) $\Rightarrow$ (c) $\Rightarrow$ (a), and so it suffices to prove (b). As usual, we may assume that $N=0$. It will certainly suffice to show that $\hat{S} \otimes_{R} N+I\left(\hat{S} \otimes_{R} M\right)$ is tightly closed in $\hat{S} \otimes_{R} M$, working over $\hat{S}$, so that there is no loss of generality in assuming that $S$ is complete. Because $R, \hat{R}$ have a common weak test element, we know from (7.12c) that $\hat{R} \otimes_{R} M=\hat{M}$ still has the property that 0 is tightly closed. Thus, we may pass to $\hat{R}$ and $\hat{M}$. But then $\hat{R}, \hat{S}$ have a common weak test element and we may apply $(7.12 \mathrm{~b})$ to obtain the desired conclusion.

To escape from the assumption that $M / N$ has finite length when $R$ is not complete, as well as to escape from the assumption that $R$ is local, we shall need either to impose the hypothesis that $N$ remain tightly closed in $M$ after arbitrary localization (this is proved in a number of special cases in [AHH]) or else to impose the hypothesis that $S$ be projective (or $\cap$-flat, defined below) over $R$. We first prove

(7.17) Theorem. Let $(R, m, K) \rightarrow(S, n, L)$ be a flat local homomorphism of Noetherian rings of characteristic $p$ such that the closed fiber $K \rightarrow S / m S$ is geometrically regular and suppose that there is an element $c \in R^{o}$ that is $a q^{\prime}$-weak test element for both $R$ and $\hat{R}$. Let $N \subseteq M$ be finitely generated $R$-modules such that $M / N$ has finite length. Then $\left(S \otimes_{R} N\right)^{*}=S \otimes_{R} N_{M}^{*}$ in $S \otimes_{R} M$. In particular, if $N$ is tightly closed in $M$ then $S \otimes_{R} N$ is tightly closed in $S \otimes_{R} M$.

Proof. The next to last statement follows from the last. We may assume that $M$ has finite length, that 0 is tightly closed in $M$, and we need only show that 0 is tightly closed in $S \otimes_{R} M$. But since $\operatorname{Ass}_{R} M=\{m\}, S$ is flat over $R$, and $m S$ is prime, it follows that $\operatorname{Ass}_{S}\left\{S \otimes_{R} M\right\}$ is $\{m S\}$. If 0 is not tightly closed in $S \otimes_{R} M$, there is an element of the tight closure whose annihilator is $m S$. It follows that we may obtain a new counterexample by localizing $S$ at $m S$. But now $n=m S$ and the extension of residue fields is separable. Since $R$ and $\hat{R}$ have a common weak test element, the result now follows from (7.16c). 
We shall say that an $R$-module $S$ (in our context, $S$ will usually be an $R$ algebra) is intersection-flat or $\cap$-flat if $S$ is flat and for every family of submodules $\left\{M_{\lambda}\right\}_{\lambda \in \Lambda}$ of every finitely generated $R$-module $M$ we have $S \otimes_{R}\left(\bigcap_{\lambda} M_{\lambda}\right) \cong$ $\bigcap_{\lambda} S \otimes_{R} M_{\lambda}$ (quite generally, there is an obvious map from the first module to the second). The $\cap$-flat modules include $R$ and are closed under arbitrary direct sum and passing to direct summands. Thus, they include the projective $R$-modules. On the other hand, if $R$ is complete local and $R \rightarrow S$ is flat local then $S$ is easily seen to be $\cap$-flat, using Chevalley's theorem. Moreover, $R[[x]]$, where $R$ is Noetherian and $x$ denotes a finite string of variables, is $\cap$-flat over $R$, since for each $N \subseteq M$ finitely generated we can identify $R[[x]] \otimes_{R} M$ and $R[[x]] \otimes_{R} N$ with $M[[x]]$ and $N[[x]]$ respectively.

We are now ready to prove one of our main results:

(7.18) Theorem. Let $h: R \rightarrow S$ be a smooth homomorphism of Noetherian rings of characteristic $p$ such that $R$ is locally excellent (or such that every local ring of $R$ contains a weak test element for its completion). Let $N \subseteq M$ be finitely generated $R$-modules.

(a) Suppose that $N_{P}$ is tightly closed in $M_{P}$ for every prime ideal $P$ of $R$. Then $\left(S \otimes_{R} N\right)_{Q}$ is tightly closed in $\left(S \otimes_{R} M\right)_{Q}$ for every prime ideal $Q$ of $S$. In particular, $S \otimes_{R} N$ is tightly closed in $S \otimes_{R} M$.

(b) Suppose that $R$ has a weak test element, that $S$ is a projective (or $\cap$ flat) $R$-module, and that $N$ is tightly closed in $M$. Then $S \otimes_{R} N$ is tightly closed in $S \otimes_{R} M$.

Proof. (a) If not, choose $Q \in \operatorname{Spec} S$ minimal such that the tight closure of $\left(S \otimes_{R} N\right)_{Q}$ is strictly larger than $\left(S \otimes_{R} N\right)_{Q}$ : assuming that there is at least one such $Q$, we can do this. It then follows that $\left(S \otimes_{R} N\right)_{Q}^{*} /\left(S \otimes_{R} N\right)_{Q}$ is killed by a power of $Q$. We can replace $S$ by $S_{Q}, R$ by $R_{P}$, where $P$ is the contraction of $Q$ to $R$, and $M, N$ by $M_{P}, N_{P}$, respectively. Changing notation, we now have that $(R, m, K) \rightarrow(S, n, L)$ is smooth local, that $N$ is tightly closed in $M$, but that $\left(S \otimes_{R} N\right)_{S \otimes_{R} M}^{*} /\left(S \otimes_{R} N\right)$ is a nonzero module of finite length. As usual, we may assume in addition that $N=0$.

The tight closure $W$ of 0 in $S \otimes_{R} M$ has finite length, and so is killed by a power of $m$. Let $W_{0}$ be $W \cap \operatorname{Ann}_{\left(S \otimes_{R} M\right)} m=W \cap\left(S \otimes_{R} V\right)$, where $V=\mathrm{Ann}_{M} m$, since $S$ is $R$-flat. If $W \neq 0$, then $W_{0} \neq 0$. On the other hand, since $R$ has a weak test element, $\bigcap_{t} N_{t}=0$, where $N_{t}=\left(m^{t} M\right)_{M}^{*}$. It follows that $\bigcap_{t}\left(N_{t} \cap V\right)=0$, and since this is a decreasing sequence of finite-dimensional vector spaces, we can choose $t$ such that $N_{t} \cap V=0$. Then $\left(S \otimes_{R} N_{t}\right) \cap\left(S \otimes_{R} V\right)=0$, and so $S \otimes_{R} N_{t}$ does not meet $W_{0}$. The image of $W_{0}$ in $\left(S \otimes_{R} M\right) /\left(S \otimes_{R} N_{t}\right) \cong S \otimes_{R}\left(M / N_{t}\right)$ will still be in the tight closure of 0 and will be nonzero. This means that $M / N_{t}$ gives a new counterexample: one in which the module is of finite length. Since $R$ contains a weak test element for $\hat{R}$, we have now contradicted (7.17). This completes the proof of part (a).

(b) Since $R$ has a weak test element, $N$ is an intersection of submodules $N^{\prime}$ of $M$ such that $M / N^{\prime}$ is of finite length and supported at a unique maximal ideal of $R$. Since $S$ is $\cap$-flat, it will suffice to show that each $S \otimes_{R} N^{\prime}$ is tightly closed in $S \otimes_{R} M$. But now part (a) applies, since each $N^{\prime}$ remains tightly closed after localization. 
(7.19) Remark. Even when $R$ is an excellent local ring or a local ring at a maximal ideal of an algebra finitely generated over an algebraically closed field, we do not know that if $I$ is tightly closed in $R$ then $I \hat{R}$ is tightly closed. It is still possible that elements of $J \hat{R}$ lie in $(I \hat{R})^{*}$, where $J \supseteq I$ consists of elements of $R$ contained in $\left(I R_{P}\right)^{*}$ for some prime $P$ of $R$ lying under a prime $Q$ in the support of $(I \hat{R})^{*} / I \hat{R}$.

(7.20) Corollary. If $R$ is an excellent local ring of characteristic $p$ and $N \subseteq M$ are finitely generated $R$-modules, then for every $R$-module $N^{\prime}$ with $N \subseteq N^{\prime} \subseteq$ $M$ that remains tightly closed under arbitrary localization, $\hat{N}_{\hat{M}}^{*} \subseteq\left(N^{\prime}\right)^{x}$. In particular, if $N$ is tightly closed in $M$ and remains so under localization, then $\hat{N}$ is tightly closed.

Proof. The first statement follows from the second, while the second statement is immediate from Theorem 7.16, since $R$ will contain a weak test element for $\hat{R}$ and $R \rightarrow \hat{R}$ is smooth.

We also have

(7.21) Corollary. Let $(R, m, K)$ be a local ring and let $N \subseteq M$ be finitely generated $R$-modules. Suppose that $\hat{N}$ is tightly closed in $\hat{M}$. Then for every smooth homomorphiswi $R \rightarrow S$ such that all maximal ideals of $S$ lie over $m$, $S \otimes_{R} N$ is tightly closed in $S \otimes_{R} M$.

Proof. A counterexample will remain a counterexample after we localize $S$ at a maximal ideal in the support of $\left(S \otimes_{R} N\right)^{*} /\left(S \otimes_{R} N\right)$. Thus, we may assume that $(R, m, K) \rightarrow(S, n, L)$ is local. As usual, we may assume that $N=0$. Since 0 is tightly closed in $\hat{M}$, it is the intersection of a decreasing sequence of submodules $\hat{N}_{t} \subseteq \hat{M}$ such that each $\hat{N}_{t}$ is tightly closed, and $\hat{M} / \hat{N}_{t}$ is of finite length. But then $\hat{N}_{t}$ is the completion of a submodule $N_{t} \subseteq M$ such that $M / N_{t} \cong \hat{M} / \hat{N}_{t}$. Since $\hat{N}_{t} \subseteq m^{h} \hat{M}$ for all $t \gg 0$ and for any $h$, we have that $N_{t} \subseteq m^{h} M$ for all $t \gg 0$ and for any $h$. It then suffices to prove that $S \otimes_{R} N_{t}$ is tightly closed in $S \otimes_{R} M$ for all $t$, for this forces the tight closure of 0 in $S \otimes_{R} M$ into $\bigcap_{h} m^{h}\left(S \otimes_{R} M\right)=0$.

But $M / N_{t}$ has finite length, and remains tightly closed over $\hat{R}$. Exactly as in the last paragraph of the proof of (7.17) we may localize $S$ at $m S$ and get a new counterexample. But then we may pass to $\hat{R}$ and $\hat{S}$ : we shall have that the maximal ideal of $\hat{R}$ expands to that of $\hat{S}$ and that the extension of residue fields is separable, and this contradicts $(7.16 \mathrm{c})$.

We next observe:

(7.22) Corollary. Let $(R, m, K) \rightarrow(S, n, L)$ be a flat local homomorphism of Noetherian rings of characteristic $p$ such that the closed fiber $S / \mathrm{mS}$ is regular and $L / K$ is separable. Suppose that $R$ is excellent. Let $N \subseteq M$ be finitely generated $R$-modules. If $N$ is tightly closed and remains so under localization and $z_{1}, \ldots, z_{d}$ are elements of $S$ that generate the maximal ideal of the fiber $S / m S$, then $S \otimes_{R} N+I\left(S \otimes_{R} M\right)$ is tightly closed in $S \otimes_{R} M$ for every ideal I generated by monomials in $z_{1}, \ldots, z_{d}$.

Proof. We replace $S$ by $\hat{S}$, which only makes the problem harder. By (7.19), $\hat{N}$ is tightly closed in $\hat{M}$, and the result follows from (7.15b). 
(7.23) Remark. We believe, but do not know, that if $(R, m, K) \rightarrow(S, n, L)$ is smooth and $K \rightarrow(S / m S)$ is separable then the induced map of completions is smooth as well. There is no problem if $L / K$ is separable or if $S$ is essentially of finite type as an $R$-algebra. However, in general, we do not know that a flat local homomorphism $(R, m, K) \rightarrow(S, n, L)$ of complete local rings is smooth if $K \rightarrow S / m S$ is geometrically regular. The problem is that we do not know that, when $R$ is a domain, one can deduce the smoothness of the generic fiber from the smoothness of the closed fiber (although it is possible to carry out the argument either if $L$ is separable over a finite purely inseparable extension of $K$ or if $R$ has a desingularization). Cf. [EGA], IV (Seconde Partie), Remarques (7.5.4)(i) and Proposition (7.9.8).

We next prove a basic result concerning when $F$-regularity is preserved by a regular morphism.

(7.24) Theorem. Let $(R, m, K) \rightarrow(S, n, L)$ be a flat homomorphism of Noetherian local rings of characteristic $p$. Suppose that $S \rightarrow \hat{S}$ is regular and that the singular locus in $S$ is closed.

If $R$ is weakly $F$-regular and the generic and closed fibers of the map $R \rightarrow S$ are regular, then $S$ is weakly $F$-regular.

In particular, if $R \rightarrow S$ is a flat local homomorphism of local rings such that $R$ is weakly $F$-regular and $S$ is excellent and the generic and closed fibers are regular, then $S$ is weakly $F$-regular.

Proof. By Theorem 7.21, there is a test element $c$ in $R$ for both $R$ and $S$. Since $R$ is weakly $F$-regular it is normal, and, hence, approximately Gorenstein (cf. [HH4] (8.5) and [Ho4]): that is, there is a decreasing sequence $\left\{I_{t}\right\}_{t}$ in $R$ of $m$-primary irreducible ideals cofinal with the powers of $m$. Choose elements $z_{1}, \ldots, z_{d} \in S$ whose images in $S / m S$ are a regular system of parameters. By (7.10c) the ideals $J_{t}=I_{t} S+\left(z_{1}^{t}, \ldots, z_{d}^{t}\right) S$ form a sequence of irreducible ideals of $S$ cofinal with the powers of $n$. To prove that $S$ is weakly $F$-regular it will suffice to show that $J_{t}$ is tightly closed for every $t$ (every ideal of $S$ is an intersection of $n$-primary irreducible ideals $J$, and each $S / J$ is embeddable in some $S / J_{t}$ ). But this is immediate from part (iv) of Theorem 7.12, since the socle in $R / I_{t}$ is one-dimensional.

We next globalize this result:

(7.25) Theorem. Let $R \rightarrow S$ be a flat homomorphism of Noetherian rings of characteristic $p$.

(a) Suppose that $\left(R^{o}\right)^{-1} S$ is regular and that $S$ is locally excellent (or that for each maximal ideal $m$ of $S$ the singular locus in $\operatorname{Spec} S_{m}$ is closed and the map from $S_{m}$ to $\left(S_{m}\right)$ is regular). If for every maximal ideal $m$ of $S$ having contraction $P$ to $R, R_{P}$ is weakly $F$-regular and $S_{m} / P S_{m}$ is regular, then $S$ is weakly $F$-regular.

(b) Suppose that $R$ is weakly $F$-regular, that $S$ is locally excellent, and that $R \rightarrow S$ has regular fibers over both minimal primes of $R$ and over closed points. Suppose that every maximal ideal of $R$ lies over a maximal ideal of $S$, which is the case if $R$ is a Hilbert ring and $S$ is finitely generated as an $R$-algebra. Then $S$ is weakly $F$-regular.

(c) Suppose that $R$ is $F$-regular, that $S$ is locally excellent, and that $R \rightarrow S$ has regular fibers. Then $S$ is $F$-regular. 
(d) Suppose that $R$ and $S$ are finitely generately algebras over a field $K$. If $R$ is weakly $F$-regular and the fibers over minimal primes and over closed points are regular, then $S$ is weakly $F$-regular.

Proof. (a) is immediate from (7.24), since the issue of whether $S$ is weakly $F$-regular is local on the maximal ideals of $S$ by Corollary (4.15) of [HH4], and the hypotheses on $S$ pass to its localizations at maximal ideals. (b) then follows at once. (c) follows from (7.24): we need to show that $S_{Q}$ is weakly $F$-regular for every prime ideal $Q$ of $S$, and if $Q$ lies over $P$ in $R$ we know that $R_{P}$ is weakly $F$-regular and that all fibers of $R_{P} \rightarrow S_{Q}$ are regular.

The conclusion in (d) follows from (b), since algebras finitely generated over a field are Hilbert rings and are excellent.

(7.26) Remark. We cannot hope to prove that if $R$ is weakly $F$-regular then $R[x]$ is weakly $F$-regular for an indeterminate $x$, unless we can prove that weakly $F$-regular implies $F$-regular. To see this, note that if $(R, m)$ is local and $P$ is a prime ideal of $R$ such that $\operatorname{dim} R / P=1$, then there are maximal ideals $n$ of $R[x]$ lying over $P$, e.g., $P R[x]+(r x-1) R[x]$ where $r$ is any element of $m-P$. If we knew that $R[x]$ is weakly $F$-regular, then since $R[x]_{n}$ is faithfully flat over $R_{P}$ it would follow that $R_{P}$ is weakly $F$-regular. Induction on $\operatorname{dim} R / P$ would then give this for all primes $P \subseteq m$.

(7.27) Remark. We now know that when $R \rightarrow S$ is a flat local homomorphism from a weakly $F$-regular ring $R$ to an excellent ring $S$ such that both the generic and closed fibers are regular, then $S$ is weakly $F$-regular. Roughly speaking, what makes this work is that a tightly closed module stays tightly closed after base change if the quotient has finite length and a one-dimensional socle. However, we should point out that under the given conditions, it is not true that tight closure commutes with base change in general: cf. Example 6.18.

(7.28) Corollary. If $R$ is a weakly $F$-regular (respectively, F-regular) local ring of characteristic $p$ and if $R$ is excellent (or the map $R \rightarrow \hat{R}$ is regular), then $\hat{R}$ is weakly $F$-regular (respectively, $F$-regular).

In Theorems 7.29 and 7.31 below we indicate some important special cases of our results on base change: in fact, in some of these cases we can improve on our earlier results. It is worth noting that there are particularly strong results concerning passage from $R$ to $L \otimes_{K} R$ when $L$ is a finite separable extension of $K$. To this end, if $R$ is a Noetherian ring of characteristic $p$, let $\tau_{q^{\prime}}(R)$ (respectively, $\left.\hat{\tau}_{q^{\prime}}(R)\right)$ denote $\left\{c \in R\right.$ : if $x \in 0^{*}$ in some finitely generated $R$-module $M$ then $c x^{q}=0$ in $F^{e}(M)$ for all $\left.q \geq q^{\prime}\right\}$ (respectively, the intersection of the contractions of $\tau_{q^{\prime}}(B)$ for local rings $B$ that are completed local rings of $R$ ). (If any element of $\tau_{q^{\prime}}(R)$ or $\hat{\tau}_{q^{\prime}}(R)$ is in $R^{o}$ then $\tau_{q^{\prime}}(R)$ (respectively, $\hat{\tau}_{q^{\prime}}(R)$ ) is the ideal generated by the $q^{\prime}$-weak (respectively, completely stable $q^{\prime}$-weak) test elements of $R$.)

(7.29) Theorem. Let $R$ be a Noetherian ring of characteristic $p$ containing a field $K$.

(a) Let $L$ be a finitely generated (respectively, an arbitrary) separable field extension of $K$, and suppose that $R$ is locally excellent (respectively, essentially of finite type over $K$ ). 
(i) If $R$ is $F$-regular, then $L \otimes_{K} R$ is $F$-regular. If $R$ is weakly $F$-regular and $L$ is algebraic over $K$ then $L \otimes_{K} R$ is weakly $F$ regular.

(ii) If $R$ has a weak test element and $N \subseteq M$ are finitely generated $R$ modules such that $N$ is tightly closed in $M$, then $\left(L \otimes_{K} R\right) \otimes_{R} N$ is tightly closed in $\left(L \otimes_{K} R\right) \otimes_{R} M$ (of course, these may be identified with $L \otimes_{K} N$ and $L \otimes_{K} M$, respectively) over $L \otimes_{K} R$.

$\left(\mathrm{a}^{o}\right)$ Let $L$ be a finite separable extension of $K$. Let $S=L \otimes_{K} R$.

(i) If $R$ is weakly $F$-regular, $F$-regular, or both $F$-rational and $a$ homomorphic image of a Cohen-Macaulay ring ( $\equiv F$-rational and Cohen-Macaulay), then so is $S$.

(ii) If $N \subseteq M$ are finitely generated $R$-modules such that $N$ is tightly closed in $M$ then $S \otimes_{R} N$ is tightly closed in $S \otimes_{R} M$. Moreover, $\tau_{q^{\prime}}(S)=\tau_{q^{\prime}}(R) S$ and $\hat{\tau}_{q^{\prime}}(S)=\hat{\tau}_{q^{\prime}}(R) S$.

(b) Let $L$ be a finite (respectively, arbitrary) purely inseparable extension field of $K$ linearly disjoint from the total quotient ring of $R$ (and such that $R$ is essentially of finite type over $K$ ) and let $P$ be a prime ideal of $R$ such that $P\left(L \otimes_{K} R_{P}\right)$ is radical (i.e., such that $L$ is linearly disjoint over $K$ from $\left.R_{P} / P R_{P}\right)$, which implies that $P\left(L \otimes_{K} R_{P}\right)$ is maximal. Then $L \otimes_{K} R_{P}$ is weakly $F$-regular.

Proof. In all parts of the theorem, when $L$ is not restricted to be finitely generated over $K$ the hypothesis that $R$ be essentially of finite type over $K$ is imposed simply to guarantee that $L \otimes_{K} R$ be Noetherian (it will be essentially of finite type over $L$ ). The case for arbitrary $L$ can then be deduced from the case where $L$ is finitely generated over $K$ by a direct limit argument: see (6.14a). Thus, we assume henceforth that $L$ is finitely generated over $K$ (and, hence, finite in case (b)).

In part (a), since $K \rightarrow L$ is separable, it is smooth, and so $R \rightarrow L \otimes_{K} R$ is smooth. Since each local ring of $L \otimes_{K} R$ is essentially of finite type over a local ring of $R, L \otimes_{K} R$ is locally excellent. (ai) now follows, in the $F$-regular case, from Theorem $(7.25 \mathrm{c})$ or from the proof of $\left(\mathrm{a}^{o} \mathrm{i}\right)$ given below, while (ai) follows, in the weakly $F$-regular case, from Theorem (7.25b). (aii) follows from part (b) of Theorem 7.18: since $L$ is free over $K, L \otimes_{K} R$ is free over $R$. We leave part $\left(\mathrm{a}^{o}\right)$ for last.

In part (b), we have that $L \otimes_{K} R$ is reduced and so may be identified with a subring of $R^{\infty}$ : thus, it is a flat purely inseparable extension of $R$, and we may apply $(6.17 \mathrm{~b})$.

It remains to prove $\left(\mathrm{a}^{o}\right)$. We first consider the final statement. Let $I$ denote $\tau_{q^{\prime}}(R)$ (respectively, $\hat{\tau}_{q^{\prime}}(R)$ ) and let $J$ denote $\tau_{q^{\prime}}(S)$ (respectively, $\hat{\tau}_{q^{\prime}}(S)$ ). First suppose that $L$ is Galois over $K$. Then $J$ is also stable under the induced action of $G=\operatorname{Gal}(L / K)$ on $L \otimes_{K} S$ and so $J=L \otimes_{K}(J \cap R)$. (This is a vector space fact: $L \otimes_{K} S$ is a direct sum of simple modules $L$ over the twisted group ring $L[G]=\sum_{g \in G} L g$ with $(\lambda g)\left(\lambda^{\prime} g^{\prime}\right)=\left(\lambda g\left(\lambda^{\prime}\right)\right)\left(g g^{\prime}\right)$, and so every $G$ stable $L$-subspace of $L \otimes_{K} S$ is a direct sum, as an $L[G]$-module, of copies of $L$, and is therefore extended). Since an element of $R$ that becomes a $q^{\prime}$ weak test element (or a completely stable one) in a faithfully flat extension must already be one, $J$ will have the form $L \otimes_{K} I_{0}$ for a certain ideal $I_{0}$ of $R$ contained in $I$. To complete the proof in the Galois case, it remains to 
show that $I \subseteq J$ when we make a finite separable extension. Before pursuing this point, we observe that when $L$ is not Galois we can consider $L^{\prime} \supseteq L$ finite Galois over $K$ (and $L$ ), and the corresponding ideal $J^{\prime}$ in $L^{\prime} \otimes_{K} R$. Then $J=J^{\prime} \cap S$ and $J^{\prime}=L^{\prime} \otimes_{K} I$ (two instances of the Galois case) so that $J=\left(L^{\prime} \otimes_{K} I\right) \cap\left(L \otimes_{K} R\right)=L \otimes_{K} I=I S$.

It remains to show that an element of $I$ remains an element of $J$. The key point is that every finitely generated $S$-module is a direct summand of a module extended from $R$. We can embed $M$ into $L[G] \otimes_{L} M$ : as an $S$-module, this is a direct sum of copies of $M$. Call this module $N$. We next claim that for each $L[G]$-module $N$, we have $N \cong L \otimes_{K} N^{G}$, where $G$ acts on $L$ canonically. For this, it suffices to show that $L[G]$, as a left module over itself, is isomorphic with a direct sum of copies of $L$ with the canonical structure. In fact, there is an $L[G]$-module isomorphism of $L \otimes_{K} L$ with $L[G]$ (where the $L[G]$-module structure on $L \otimes_{K} L$ is induced by the canonical structure on the first copy of $L$ ) sending $\lambda \otimes \mu$ to $\sum_{g \in G} \lambda g(\mu) g \in L[G]$. Cf. [SGA4 $\frac{1}{2}$ ], p. 14. The fact that this map of $L$-vector spaces has rank $|G|$ is equivalent to the fact that the elements of $G$, thought of as functions from $L$ to $L$, are linearly independent over $L$. Since $N=L[G] \otimes_{K} M$ the action of $G$ on $N$ is $R$-linear, and so $N^{G}$ is an $R$-module. In fact, $N^{G} \cong\left(L[G]^{G}\right) \otimes_{K} M=(L \beta) \otimes_{K} M$ where $\beta=\sum_{g \in G} g:$ as an $R$-module this is isomorphic with ${ }_{R}\left(L \otimes_{K} M\right) \cong M^{|G|}$.

It is now quite easy to see that an element $c \in \tau_{q^{\prime}}(R)$ is in $\tau_{q^{\prime}}(S)$ for $S=L \otimes_{K} R$ : the point is that since every $S$-module is a direct summand over $S$ of one of the form $L \otimes_{K} M$, where $M$ is an $R$-module, it suffices to check the defining condition on modules of the form $L \otimes_{K} M$, where the tight closure of 0 is $L \otimes_{K} 0_{M}^{*}$.

Finally, suppose that $c \in \hat{\tau}_{q^{\prime}}(R)$. Suppose that $Q$ is a prime ideal of $S$ lying over $P$ in $R$. We know that $c \in \tau_{q^{\prime}}\left(\left(R_{P}\right)^{\wedge}\right)$. Now $\left(S_{Q}\right)^{\wedge}$ is a local ring, at one of maximal ideals, of $L \otimes_{K}\left(R_{P}\right)^{r}$. Thus, it suffices to show that if $(R, m, \Omega)$ is a complete local ring containing a field $K, L$ is a finite separable extension of $K$, and $c \in \tau_{q^{\prime}}(R)$, then for each maximal ideal $Q$ of $S$ if $T=\left(L \otimes_{K} R\right)_{Q}$ then $c \in \tau_{q^{\prime}}(T)$.

Let $L=K[z] / f(z) K[z] . L \otimes_{K} R$ is a product of local rings and $T$ is one of the factors: in fact the maximal ideals in $L \otimes_{K} R$ correspond to the irreducible factors of $f$ over the residue field $\Omega$ of $R$, the factorization lifts to $R$, and so $T$ will have the form $R[z] / g(z) R[z]$ where $g$ is a monic polynomial that is irreducible and separable modulo $m$. Now $m T$ is the maximal ideal of $T$ and $\Omega^{\prime}=T / m T \cong \Omega[z] / \bar{g}(z) \Omega[z]$ is a separable extension of $\Omega$. It follows from the discussion in the final paragraph of (7.11) that $T \cong \Omega^{\prime} \otimes_{\Omega} R$ and so we may apply the result already established for ordinary test elements when one tensors with a finite separable extension of a subfield to conclude that $c \in \tau_{q^{\prime}}(T)$.

Now suppose that $N$ is tightly closed in $M$. It is clear that if $L^{\prime} \otimes_{K} N$ is tightly closed in $L^{\prime} \otimes_{K} M$ for a larger field $L^{\prime}$ then $L \otimes_{K} N$ will be tightly closed in $L \otimes_{K} M$. Hence, we may assume, as above, that $L$ is Galois over $K$ with Galois group $G$. If $d \in S^{o}$ is used in a given tight closure test we may use $\prod_{g \in G} g(d) \in R^{o}$ instead. Thus, we may perform all tight closure tests over $S$ with test elements in $R^{o}$. It follows easily that $\left(L \otimes_{K} N\right)^{*} \cap M=N$. But, since $\left(L \otimes_{K} N\right)^{*}$ is a $G$-stable $L$-vector space we have that $\left(L \otimes_{K} N\right)^{*}=$ $L \otimes_{K}\left(\left(L \otimes_{K} N\right)^{*} \cap M\right)=L \otimes_{K} N$. 
Now suppose that $R$ is weakly $F$-regular. We know from the argument above that every $S$-module is a direct summand of an extended $R$-module. Since 0 will be tightly closed in every module of the form $S \otimes_{R} M$, it is clear that $S$ is weakly $F$-regular. If $R$ is $F$-regular, we note that each local ring $S_{Q}$ of $S$ is the local ring of $L \otimes_{K} R_{P}$, where $P$ is the contraction of $Q$, at a maximal ideal. Since $R_{P}$ is weakly $F$-regular, so is $L \otimes_{K} R_{P}$, and the result follows. Finally, if $R$ is $F$-rational, C-M, and $n$ is any maximal ideal of $S$ lying over $m$ in $R$, we may choose $x_{1}, \ldots, x_{d} \in m$ so that they form a system of parameters in $R_{m}$. The ideal $\left(x_{1}, \ldots, x_{d}\right) R_{m}$ is tightly closed in $R_{m}$, and remains so in $L \otimes_{K} R_{m} \cong S_{m}$. Since it is generated by a regular sequence it remains tightly closed when we localize further to $S_{n}$ (cf. (4.5)). Since the $x_{j}$ form a system of parameters for $S_{n}$, we see that $S_{n}$ is $F$-rational, and $n$ was arbitrary.

Remark. We want to emphasize that we do not know, even for a weakly $F$ regular algebra $R$ finitely generated over a field $K$, that $S=L \otimes_{K} R$ is again weakly $F$-regular. We do not know this even when $L=K(t)$, a pure transcendental extension of transcendence degree one. The problem is that maximal ideals of $S$ may lie over prime ideals of $R$ that are not maximal. In fact, if we knew that $S$ is weakly $F$-regular when we make a pure transcendental extension of transcendence degree one, it would follow that every weakly $F$-regular finitely generated $K$-algebra is $F$-regular. For, by induction, we would get this result for arbitrary pure transcendental extension fields of finite transcendence degree, and then by a direct limit argument it would follow, using (6.14a), for all pure transcendental field extensions. The result of M. P. Murthy given in (8.1) would then suffice to show that the original ring $R$ must have been $F$-regular. We are grateful to Murthy for pointing out an error in an earlier version of this manuscript, as well for allowing us to communicate the arguments given in (8.1).

(7.30) Discussion. The proof of Theorem 7.31 below is based on part (b) of Theorem 7.18. However, a good deal of insight can be achieved by utilizing instead the fact that polynomial and power series rings have many automorphisms, and an argument based on this idea is given following the proof of Theorem 7.31.

Let $R$ be a Noetherian ring containing infinitely many units $\alpha_{j}$ such that if $i \neq j$ then $\alpha_{i}-\alpha_{j}$ is a unit. This condition is satisfied whenever $R$ contains an infinite field or a local ring with an infinite residue field.

Let $x$ denote a string $x_{1}, \ldots, x_{n}$ of indeterminates over $R$. Let $S=R[x]$ or $R[[x]]$. Let $M$ be a finitely generated $R$-module. The $R$-automorphisms $\theta$ of $S$ induce $R$-linear maps from $S \otimes_{R} M$ to itself (we may also think of $S \otimes_{R} M$ as $M[x]$ or $M[[x]]$, as the case may be). Suppose that a submodule $W$ of $S \otimes_{R} M$ is stable under all these maps. We want to make the observation that if $\sum_{\nu} u_{\nu} x^{\nu} \in W$, then each $u_{\nu} x^{\nu} \in W$. (Here, $\nu$ denotes a variable $n$-tuple of nonnegative integers and the sum is finite in the polynomial case and possibly infinite in the power series case.) By induction on the number of variables, we may reduce to the case $n=1$.

Now, for every unit $\alpha \in R$ there is a unique (continuous in the power series case) $R$-automorphism $\theta_{\alpha}$ of $S$ sending $x$ to $\alpha x$ : if $f=\sum_{i} r_{i} x^{i}$ then $\theta_{\alpha}(f)=\sum_{i} r_{i} \alpha^{i} x^{i} \in J$. The induced map on $S \otimes_{R} M$ sends $w=\sum_{i} u_{i} x^{i}$ to $w_{\alpha}=\sum_{i} \alpha^{i} u_{i} x^{i}$. 
Fix a positive integer $N$ and choose $\alpha_{0}, \ldots, \alpha_{N}$ to be units of $R$ such that if $i \neq j$ then $\alpha_{i}-\alpha_{j}$ is a unit of $R$ : this is possible because of our hypothesis on $R$. If $w=\sum_{i} u_{i} x^{i} \in W$, then every $w_{\alpha}=\sum_{i} \alpha^{i} u_{i} x^{i} \in W$, since $w_{\alpha}=\theta_{\alpha}(w)$. Applying this with $\alpha=\alpha_{0}, \ldots, \alpha_{N}$ we find that $\sum_{i=0}^{N} \alpha_{j}^{i}\left(u_{i} x^{i}\right) \in$ $W+x^{N+1}\left(S \otimes_{R} M\right)$ for all $j$. Since the size $N+1$ Vandermonde matrix $\left(\alpha_{j}^{i}\right)$ is invertible, all the elements $u_{i} x^{i}$ are in $W+x^{N+1}\left(S \otimes_{R} M\right)$ for $i \leq N+1$. But then every $u_{i} x^{i}$ is in $W+x^{N+1}\left(S \otimes_{R} M\right)$ for all $N$, and it follows that every $u_{i} x^{i} \in W$.

(7.31) Theorem. Let $R$ be a locally excellent Noetherian ring of characteristic $p$. Let $x$ denote a string of indeterminates $x_{1}, \ldots, x_{n}$.

(a) If $R$ is weakly $F$-regular then $R[[x]]$ is weakly $R$-regular. If $R$ is weakly $F$-regular and a Hilbert ring then $R[x]$ is weakly $F$-regular.

(b) If $R$ is $F$-regular then $R[x]$ is $F$-regular.

(c) Suppose that $R$ has a weak test element. If $S=R[x]$ or $R[[x]]$ and $N \subseteq M$ are finitely generated $R$-modules such that $N$ is tightly closed. in $M$, then $S \otimes_{R} N$ is tightly closed in $S \otimes_{R} M$.

Proof. Since $R \rightarrow R[x]$ is smooth, the statements in parts (a) and (b) about the polynomial ring case are immediate from parts (b) and (c) of Theorem 7.25. To complete the proofs of (a) and (b), it only remains to check the statement about $R[[x]]$ in part (a). Every maximal ideal $Q$ of $R[[x]]$ can be written as $m R[[x]]+(x) R[[x]]$, where $m$ is a maximal ideal of $R$. It suffices to show that the completion of $R[[x]]_{Q}$ is weakly $F$-regular for each such $Q$. The completion is isomorphic to $\left(R_{m}\right)^{\wedge}[[x]]$. Since $R_{m}$ is excellent, it has a weak test element, and so $\left(R_{m}\right)$ is weakly $F$-regular. The result now follows from part (a) of Theorem 7.25.

It remains to establish (c). In the polynomial ring case this is immediate from Theorem (7.18b). In the power series case the fact that $R[[x]]$ is $\cap$-flat over $R$ together with the fact that $R$ has a weak test element enable us to reduce to the case where $M / N$ is killed by a power of a maximal ideal $m$ of $R$. As usual, we may assume that $M=M / N$ and 0 is tightly closed. This remains true when we replace $R$ by $\left(R_{m}\right)^{\wedge}$. If 0 is tightly closed in $M[[x]]$ over $\left(R_{m}\right)^{\wedge}[[x]]$ this is certainly true over $R[[x]]$. Thus, we may assume that $R$ is a complete local ring, so that $R \rightarrow R[[x]]$ is smooth, and the result now follows from part (b) of Theorem (7.18).

We now comment on an alternative partial proof for part (c) in the case where $R$ contains an infinite field $K$. It then follows from (7.30) that whenever $\sum_{\nu} u_{\nu} x^{\nu} \in 0^{*}$ so does each $u_{\nu} x^{\nu}$. Thus, it suffices to show that if $u x^{\nu}$ is in $0^{*}$ in $M[x]$ or $M[[x]]$ then $u$ is in $0_{M}^{*}$. If there is a counterexample we can preserve the fact that $u$ is not in $0_{M}^{*}$ while replacing $R$ by the completion of its localization at a suitable maximal ideal. The result is now clear, since we may use a weak test element $c \in R^{o}$, and if $c u^{q}=0$ in $F_{S}^{e}\left(S \otimes_{R} M\right) \cong$ $S \otimes_{R} F^{e}(M) \cong F^{e}(M)[x]$ or $F^{e}(M)[[x]]$, then $c u^{q}=0$ in $F^{e}(M)$.

Our next objective is to record improved versions of the results of $\S 6$. Theorem 7.32 below strengthens $(6.1)$.

(7.32) Theorem. Let $R$ be a Noetherian ring of characteristic $p$.

(a) Let $(R, m, K)$ be a local ring such that $R \rightarrow \hat{R}$ has regular fibers and 
let $c \in R^{o}$ be any element such that $\left(R_{\mathrm{red}}\right)_{c}$ is $F$-regular and Gorenstein. Then $c$ has a power which is a completely stable $q^{\prime}$-weak test element for $R$. If $R$ is reduced, then $c$ has a power which is a completely stable test element for $R$.

(b) Let $R$ be an algebra of finite type over a local ring $(B, m, K)$ such that $B \rightarrow \hat{B}$ is smooth (e.g., such that $B$ is excellent). Let $c$ be an element of $R^{o}$ such that $\left(R_{\mathrm{red}}\right)_{c}$ is $F$-regular and Gorenstein. Then $c$ has a power which is a completely stable weak test element for $R$. If $R$ is reduced then $c$ has a power which is a completely stable test element for $R$.

Proof. We can argue precisely as in the proof of Theorem 6.1: we pass to the reduced case, and then we need to see that $(\hat{R})_{c}$ (respectively, for $(b)$, that $\left.\left(R \otimes_{B} \hat{B}\right)_{C}\right)$ is $F$-regular and Gorenstein. Since $R \rightarrow \hat{R}$ has regular fibers (respectively, $B \rightarrow \hat{B}$ is smooth and, hence, $R \rightarrow R \otimes_{B} \hat{B}$ is regular) we have that $\left(R \otimes_{B} \hat{B}\right)_{c}$ is $F$-regular (since $R_{c}$ is, and we may apply part (c) of Theorem 7.25) and Gorenstein (since $R_{c}$ is, and so are the fibers).

We also obtain an improved version of Theorem 6.21 on the existence of common test elements. (Likewise, Theorem 6.22 is valid with the weakened hypothesis that $\left(R^{o}\right)^{-1} S_{\text {red }}$ be $F$-regular and Gorenstein instead of regular.)

(7.33) Theorem. Let $(R, m, K) \rightarrow(S, n, L)$ be a flat map of local rings such that for some $c \in R^{o},\left(S_{\mathrm{red}}\right)_{c}$ is $F$-regular and Gorenstein. (There will be such a $c$ if $\left(R^{o}\right)^{-1} S_{\mathrm{red}}$ is $F$-regular and Gorenstein and the locus which fails to satisfy these conditions is closed in $S_{\text {red. }}$ ) Suppose that $S \rightarrow \hat{S}$ has regular fibers. Then $c$ has a power that is a completely stable $q^{\prime}$-weak test element in all of the rings $R, \hat{R}, S$, and $\hat{S}$. If $S$ is reduced then $c$ has a power that is a completely stable test element in all of the rings $R, \hat{R}, S$, and $\hat{S}$.

Proof. The argument is the same as for (6.21): we can pass to the reduced case, and we then observe that $(\hat{S})_{c}$ is $F$-regular and Gorenstein. The rest of the argument is the same as for $(6.21)$.

(7.34) Corollary. Let $(R, m, K)$ be a reduced local ring such that for every $x \in m, R_{x}$ is Gorenstein and $F$-regular. Suppose that $R$ is excellent (or that $R \rightarrow \hat{R}$ has regular fibers). Then $R$ has an m-primary ideal whose intersection with $R^{o}$ consists of test elements (thus, $\tau(R)$ is m-primary).

Proof. This is immediate from (7.32).

(7.35) Discussion. If $(S, n, L)$ is a local ring then $\tau(S)$ is the intersection of the annihilators of $0_{M}^{*}$ as $M$ runs through all $S$-modules of finite length. $S$ has a test element, iff some element of $\tau(S)$ is in $S^{o}$, i.e., iff $\tau(S)$ is not contained in any minimal prime if $S$, in which case $\tau(S)$ is the same as the ideal generated by all the test elements. The test elements in $S$ always coincide with $\tau(S) \cap S^{o}$. See [HH4], (8.23) and its proof, where it is also shown that one need only let $M$ run through all finite length submodules of $M$ which are essential extensions of a copy of $K$. Moreover, it suffices to let $M$ run through any family of finite length essential extensions of $K$ whose union is the injective hull of $K$, since every finite length essential extension will be embeddable in one of these.

We next observe: 
(7.36) Theorem. Let $(R, m, K) \rightarrow(S, n, L)$ be a flat local homomorphism of complete local rings such that $L / K$ is separable and the closed fiber $S / m S$ is regular. Then $\tau(S)=\tau(R) S$.

Proof. Let $E$ be an injective hull for $K$ over $R$ and let $M_{t}$ denote $A n_{E} m^{t}$, which may be identified with an injective hull for $K$ over $R / m^{t}$. Let $z_{1}, \ldots$, $z_{d}$ be elements of $n$ whose images in $S / \mathrm{mS}$ are a regular system of parameters. Let $J_{t}=\left(z_{1}^{t}, \ldots, z_{d}^{t}\right)$ and $W_{t}=\left(S / J_{t}\right) \otimes_{R} M_{t}$. Then by $(7.10 \mathrm{~d}), \underline{\lim } W_{t}$ is an injective hull for $L$ over $S$, and by (7.35), $\tau(S)=\bigcap_{t} \operatorname{Ann}_{S} 0_{W_{t}}^{*}$. By part (b) of Theorem 7.12, $0_{W_{t}}^{*}=\left(S / J_{t}\right) \otimes_{R}\left(0_{M_{t}}^{*}\right)$. Let $\mathfrak{B}_{t}=\operatorname{Ann}_{R}\left(0_{M_{t}}^{*}\right)$. Since $S / J_{t}$ is $R$-flat by $(7.10 \mathrm{~b})$, the annihilator of $\left(S / J_{t}\right) \otimes_{R}\left(0_{M_{t}}^{*}\right)$ in $S / J_{t}$ is $\mathfrak{B}_{t}\left(S / J_{t}\right)$, and it follows that the annihilator of $\left(S / J_{t}\right) \otimes_{R}\left(0_{M_{t}}^{*}\right)$ in $S$ is $\mathfrak{B}_{t} S+J_{t}$. Thus, $\tau(S)=\bigcap_{t}\left(\mathfrak{B}_{t} S+J_{t}\right)=\bigcap_{t, h}\left(\mathfrak{B}_{t} S+J_{h}\right)=\bigcap_{t}\left(\bigcap_{h}\left(\mathfrak{B}_{t} S+J_{h}\right)\right)=\bigcap_{t} \mathfrak{B}_{t} S$. Now, by (7.35), $\tau(R)=\bigcap_{t} \mathfrak{B}_{t}$. Since $R$ is complete, by Chevalley's theorem, for every positive integer $N, \mathfrak{B}_{t} \subseteq \tau(R)+m^{N}$ for $t \gg 0$, and it follows that for every $N>0, \tau(R) S=\left(\bigcap_{t} \mathfrak{B}_{t}\right) S \subseteq \bigcap_{t}\left(\mathfrak{B}_{t} S\right) \subseteq \tau(R) S+m^{N} S$, which implies that $\tau(R) S=\left(\cap_{t} \mathfrak{B}_{t} S\right)$, which is $\tau(S)$.

(7.37) Remark. So far as we know, it may be true that whenever $(R, m, K) \rightarrow$ $(S, n, L)$ is a flat local homomorphism such that $R$ is complete, $S / m S$ is geometrically regular over $K$, and $\left(S_{\text {red }}\right)_{d}$ is regular for some $d \in R^{o}$, then $\left(z_{1}, \ldots, z_{h}\right) M$ is tightly closed in $M$ for every finite length module $M$ in which 0 is tightly closed, where the $z$ 's are any elements in $n$ whose images in $S / m S$ are a system of parameters. (We are no longer assuming that $L / K$ is separable.) If this is true, one obtains by exactly the same proof given for Theorem 7.36 that if $(R, m, K) \rightarrow(S, n, L)$ is a flat local homomorphism such that $R$ is complete, $S / m S$ is geometrically regular over $K$, and $\left(S_{\mathrm{red}}\right)_{d}$ is regular for some $d \in R^{o}$ (this is needed to insure the existence of a common test element), then $\tau(S)=\tau(R) S$. Once this is known it will also follow that if $c$ is a completely stable test element in $R, S$ is excellent, and $R \rightarrow S$ is smooth, then $c$ is a completely stable test element in $S$. The point is that one needs to see that for every prime $Q$ of $S$, the image of $c$ is a test element in $\left(S_{Q}\right)^{)}$, and one knows that the image of $c$ is a test element in $\left(R_{P}\right)$, where $P$ is the contraction of $Q$ to $R$. At this point one does not know that $\left(R_{P}\right)^{\wedge} \rightarrow\left(S_{Q}\right)^{r}$ is smooth (see Remark 7.23), but we do not need this: only that there exists $d \in\left(R_{P}\right)^{o}$ such that $\left(\left(S_{Q}\right)_{\text {red }}\right)_{d}$ is regular and that the closed fiber is geometrically regular. Since

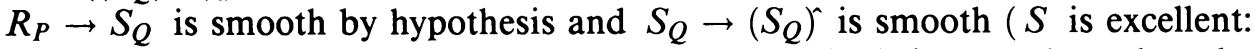
this is the only part of excellence we need), $R_{P} \rightarrow\left(S_{Q}\right)^{r}$ is smooth, and so the closed fiber, which is the same as the closed fiber of the map $\left(R_{P}\right)^{r} \rightarrow\left(S_{Q}\right)$, is geometrically regular. Since we can choose $d \in\left(R_{P}\right)^{o}$ such that $\left(\left(R_{P}\right)_{\text {red }}\right)_{d}$ is regular and since $\left(R_{P}\right)_{\text {red }} \rightarrow\left(S_{Q}\right)_{\text {red }}$ is smooth, we have that $\left(\left(S_{Q}\right)_{\text {red }}\right)_{d}$ is regular. Since $S_{Q} \rightarrow \hat{S}_{Q}$ is smooth, $\left(\left(S_{Q}\right)_{\text {red }}\right)_{d}$ is regular. Thus, the image of $c$ is a completely stable test element in $S$.

Although we cannot, in general, handle the situation where fibers are geometrically $F$-regular rather than regular, we can handle products in certain cases. We shall discuss both ordinary tensor products of algebras of finite type over a field and complete tensor products of complete local rings with a common coefficient field. In both cases, the key to the theory is the existence, under 
certain hypotheses, of test elements of the form $c \otimes d$ or $c \hat{\otimes} d$. We need some preliminary lemmas:

(7.38) Lemma. Let $R$ be a complete local ring.

(a) Suppose that $R$ has an infinite residue field and let $\mathcal{P}$ be any property of rings. Let $S=R\left[\left[x_{1}, \ldots, x_{n}\right]\right]$. Let $J$ be the radical defining ideal of the closure of the set of primes $Q$ of $S$ such that $S_{Q}$ has property $\mathcal{P}$. Then $J$ is either the maximal ideal of $S$ or else has the form IS, where $I$ is a radical ideal of $R$.

(b) Suppose that $R$ is equicharacteristic. Then the homomorphism $R \rightarrow$ $R\left[\left[x_{1}, \ldots, x_{n}\right]\right]$ has regular fibers.

(c) Suppose that $R$ has a perfect coefficient field $K \subseteq R$, and let $L \supseteq K$ be a field. Then the fibers of the map $R \rightarrow L[[R]]$ are geometrically regular.

Proof. (a) $J$ is a radical ideal of $S$ invariant under all automorphisms of $S$. We shall prove that such an ideal has one of the two forms specified. We first consider the case $n=1$, so that $S=R[[x]]$. Let $f=\sum_{i=0}^{\infty} r_{i} x^{i}$ be any element of $J$. By the discussion in (7.30), we know that every $r_{i} x^{i} \in J$, and since $J$ is radical, we see that $r_{i} x \in J$ for $i \geq 1$ and it follows that $J$ has the form $I S+I^{\prime} x S$ where $I \subseteq I^{\prime}$ are ideals of $R$. For every element $u \in m$, the maximal ideal of $R$, there is a continuous automorphism of $R[[x]]$ that fixes $R$ and sends $x$ to $x-u$. It follows that $I S+I^{\prime}(x-u) S=I S+I^{\prime} x S$ and so $m I^{\prime} \subseteq I$. If $I^{\prime}=R$ then $m \subseteq I$ and so $J$ is either the maximal ideal of $S$ or $S$ itself. If $I^{\prime} \subseteq m$ we have that $I^{\prime 2} \subseteq m I^{\prime} \subseteq I \subseteq I^{\prime}$, so that $\operatorname{Rad} I^{\prime}=\operatorname{Rad} I$. Since $I=J \cap R$ is radical, we must have $I^{\prime}=I$ and $J=I S$.

Now suppose that $n>1$. Since every automorphism of

$$
R^{\prime}=R\left[\left[x_{1}, \ldots, x_{n-1}\right]\right]
$$

extends to $S=R^{\prime}\left[\left[x_{n}\right]\right], J^{\prime}=J \cap R^{\prime}$ is a radical ideal stable under every automorphism of $R^{\prime}$. If $J$ is not the maximal ideal of $S$ then $J=J^{\prime} S$ where $J^{\prime}$ is either of the form $I R^{\prime}$ or else $J^{\prime}$ is the maximal ideal $Q^{\prime}$ of $R^{\prime}$. But $Q^{\prime} S$ is not stable under the automorphisms of $S$ which permute the $x_{i}$. It follows that $J=I S$.

(b) For this it suffices to show that when $R$ is a domain the generic fiber is regular, and it is harmless to replace $R$ by a module-finite extension domain $R^{\prime}$, since the new generic fiber is faithfully flat over the original. If the coefficient field is characteristic 0 or perfect we can choose $R^{\prime}$ so that it is the integral closure of a regular ring in a separable finite algebraic extension of its fraction field, and this proves the result. In characteristic $p$, if the residue field is infinite, the radical ideal $J$ defining the singular locus in $S=R\left[\left[x_{1}, \ldots, x_{n}\right]\right]$ is nonzero and has the form $I S$ where $I$ is an ideal of $R$ or else is the maximal ideal of $S$, by (a). We may assume that $R$ is not a field. We then see that $J$ must contain a nonzero element of $R$, as required.

(c) Let $F$ be a finite algebraic field extension of the fraction field of $R / P$, where $P$ is a prime ideal of $R$. We must show that $F \otimes_{R} L[[R]]$ is regular. Choose a domain $D$ module-finite over $R$ with fraction field $F$. Then $F \otimes_{R}$ $L[[R]] \cong F \otimes_{D}\left(D \otimes_{R} L[[R]]\right) \cong F \otimes_{D}(L[[D]])$. Thus, we may assume that $R$ is a domain with fraction field $F$, and it suffices to show that $F \otimes_{R} L[[R]]$ is regular. $R$ is module-finite over a regular ring $A=K[[x]]$, where $x$ indicates a string $x_{1}, \ldots, x_{n}$, and we have that $F \otimes_{R} L[[R]] \cong F \otimes_{A} L[[A]]$. We can find a 
finite algebraic field extension $F^{\prime}$ of $F$ such that $F^{\prime}$ is a separable extension of $K\left[\left[x^{1 / q}\right]\right]$ for some $q=p^{e}$ (since $K$ is perfect). Since enlarging $F$ to $F^{\prime}$ only makes the problem harder and since the separable part of the field extension will not disturb regularity, we have reduced to the case where $F$ is the fraction field of $K\left[\left[x^{1 / q}\right]\right]$. But then $F \otimes_{R} L[[R]]$ is a localization of $K\left[\left[x^{1 / q}\right]\right] \otimes_{R} L[[x]]$ with $R=K[[x]]$, and this is $L\left[\left[x^{1 / q}\right]\right]$, which is regular.

(7.39) Discussion. We want to discuss the complete tensor product $R \hat{\otimes}_{K} S$ in the situation where $\left(R, m, K^{\prime}\right)$ and $(S, n, L)$ are complete local rings, $K \subseteq R$ is a subfield such that the residue field $K^{\prime}$ of $R$ is a finite algebraic extension of $K$, and $K$ is an arbitrary subfield of $S$. More generally, we want to discuss $M \hat{\otimes}_{K} N$ where $M$ is a finitely generated $R$-module and $N$ is a finitely generated $S$-module. We shall take $M \hat{\otimes}_{K} N$ to be the completion of $M \otimes_{K} N$ with respect to the ideal $J=m \otimes_{K} S+R \otimes_{K} n$. Note that $\left(R \otimes_{K} S\right) / J \cong K^{\prime} \otimes_{K} L$ is finitedimensional as an $L$-vector space and is an Artin ring (which is a complete intersection over $L$ ). It follows that $R \hat{\otimes}_{K} S$ is a semilocal Noetherian ring which is local if $K^{\prime} \otimes_{K} L$ is a field (e.g., if $K^{\prime}=K$ ). If $u_{1}, \ldots, u_{r}$ generate $M$ and $v_{1}, \ldots, v_{s}$ generate $N$ it is easy to see that the images of the $u_{i} \otimes v_{j}$ generate $M \hat{\otimes}_{K} N$ as a module over $R \otimes_{K} S$. If $R$ is module-finite over a complete local ring $A$ containing $K$ and $S$ is module-finite over a complete local ring $B$ containing $K$, then we may identify $R \hat{\otimes}_{K} S \cong R \otimes_{A}\left(A \hat{\otimes}_{K} B\right)_{B} \otimes S$ and, more generally, $M \hat{\otimes}_{K} N \cong M \otimes_{A}\left(A \hat{\otimes}_{K} B\right)_{B} \otimes N$. When $S=L$, a field containing $K$, and $K^{\prime}=K$, we may identify $R \hat{\otimes}_{K} L$ with $L[[R]]$ discussed in (6.3). When $K^{\prime}$ and $L$ are both finite algebraic extensions of $K$, our notion of complete tensor product coincides with the one given by Serre [S], Chapitre $\mathrm{V}, \mathrm{A} 2$.

Note that we can always represent $R$ as a finite module over $A=K[[x]]$, where $x$ denotes a string of formal indeterminates. In this case, $A \hat{\otimes}_{K} S \cong$ $S[[x]]$, and $R \hat{\otimes}_{K} S \cong R \otimes_{A}(S[[x]])$. Moreover, $R \hat{\otimes}_{K} S$ is (faithfully) flat over both $R$ and $S$ : in the case of $R$, one may see this by using the fact that it is clear when $R=A=K[[x]]$ and making a base change. In the case of $S$, one reduces to showing that $R \hat{\otimes}_{K}$ is an exact functor on finitely generated $S$-modules: this follows from the fact that $\left(R / m^{t}\right) \otimes_{K}$ is exact for all $t$, along with the observation that $\left(R \hat{\otimes}_{K} N\right) / m^{t}\left(R \hat{\otimes}_{K} N\right) \cong\left(R / m^{t}\right) \otimes_{K} N$.

We also note that $R \hat{\otimes}_{K}$ and $\hat{\otimes}_{K} S$ are faithfully flat functors from finitely generated $R$-modules and finitely generated $S$-modules, respectively, to finitely generated $\left(R \hat{\otimes}_{K} S\right)$-modules (one may also think of these functors as $\left(R \otimes_{K} S\right) \otimes_{R}$ and $\left(R \hat{\otimes}_{K} S\right) \otimes_{S}$, respectively). Moreover, $M \hat{\otimes}_{K} N \cong\left(M \hat{\otimes}_{K} S\right) \otimes_{R \hat{\otimes}_{K} S}\left(R \hat{\otimes}_{K} N\right)$.

(7.40) Lemma. Let $R$ and $S$ be Noetherian rings of characteristic $p$.

(a) Let $R$ be an algebra finitely generated over a field $K$ such that the total quotient ring $\mathcal{T}$ of $R$ is separable over $K$ (i.e., a product of separable field extensions). Then $U=\left\{P \in \operatorname{Spec} R: R_{P}\right.$ is geometrically regular over $K\}$ is Zariski open in $\operatorname{Spec} R$, and contains the minimal primes of $R$. Hence, there is an element $c \in R^{o}$ such that $R_{c}$ is geometrically regular over $K$.

(b) Let $(R, m, K)$ be complete, reduced, local with coefficient field $K$ such that for every $q=p^{e}, K^{1 / q}[[R]]$ is reduced (an equivalent statement is that $K^{\infty}[[R]]$ is reduced). Then there exists an element $c \in R^{o}$ such that $K^{1 / q}[[R]]_{c}$ is geometrically regular over $K^{1 / q}$ for all $q$. 
(c) Let $(R, m, K)$ be a complete local ring with coefficient field $K$. Let $D$ be a complete local domain with coefficient field $L \supseteq K$, let $D^{\prime}$ be any complete local domain between $D$ and $D^{1 / q}$ for fixed $q$, and let $\mathcal{F}, \mathcal{F}^{\prime}$ be the fraction fields of $D, D^{\prime}$ respectively. Then $\mathcal{F}^{\prime} \otimes_{D^{\prime}}\left(R \hat{\otimes}_{K} D^{\prime}\right)$ is faithfully flat over $\mathcal{F} \otimes_{D}\left(R \hat{\otimes}_{K} D\right)$.

(d) Let $R, S$ be complete local rings with coefficient fields $K, L$ (respectively, finitely generated algebras over fields $K, L)$. Suppose that $K \subseteq L$. Let the symbol " $\otimes$ " denote complete tensor product $\hat{\otimes}_{K}$ over $K$ in the first case (cf. (7.39)).(respectively, the usual tensor product $\otimes_{K}$ over $K$ ). Assume that $K^{1 / q}[[R]]$ is reduced for all $q=p^{e}$ (respectively, that the fraction field of $R$ is separable over $K$; this is equivalent to the assertion that $K^{1 / q} \otimes_{K} R$ is reduced for all $q$ ). Let $c \in R^{o}$ be such that $K^{1 / q}[[R]]_{c}$ is geometrically regular over $K^{1 / q}$ for all $q$ (respectively, $R_{c}$ is geometrically regular over $K)$. Let $d \in S^{\circ}$ be such that $S_{d}$ is regular. Then $(R \otimes S)_{c \otimes d}$ is regular.

Proof. (a) is a well-known consequence of the Jacobian criterion (cf. [N], §46).

(b) Let $K^{\infty}\langle R\rangle$ denote $\bigcup_{q} K^{1 / q}[[R]]$, which by Lemma 6.6 is an excellent ring with completion $K^{\infty}[[R]]$. Since $K^{\infty}\langle R\rangle \rightarrow K^{\infty}[[R]]$ is smooth, one is reduced iff the other is. The defining ideal of the regular locus in $K^{\infty}\langle R\rangle$ cannot be contained in any minimal prime of $K^{\infty}\langle R\rangle$. Let $c$ be an element of $K^{\infty}\langle R\rangle^{\circ}$ in this ideal. Since $K^{\infty}\langle R\rangle$ is purely inseparable over $R$, we may replace $c$ by a power which is actually in $R^{o}$. Thus, $c \in R^{o}$ and $K^{\infty}\langle R\rangle_{c}$ is regular. We claim that $K^{1 / q}[[R]]_{c}$ is geometrically regular over $K^{1 / q}$ for all $q$. For if $L$ is a finite purely inseparable extension of $K^{1 / q}$ then $L \subseteq K^{1 / q^{\prime}}$ for some $q^{\prime} \geq q$ and $L \otimes_{K}\left(K^{1 / q}[[R]]\right)$ (subscripted $\otimes$ is the usual tensor product) $\cong L[[R]]$. Since $L[[R]] \rightarrow K^{1 / q^{\prime}}[[R]] \rightarrow K^{\infty}\langle R\rangle$ is faithfully flat, so is the result of localizing at $c$. Since $K^{\infty}\langle R\rangle_{c}$ is regular, so is $L[[R]]_{c}$.

(c) The ring $R$ is module-finite over a formal power series ring $B=K[[y]]$. Then $\mathcal{F}^{\prime} \otimes_{D^{\prime}}\left(R \hat{\otimes}_{K} D^{\prime}\right) \cong R \otimes_{B}\left(\left(\mathcal{F}^{\prime} \otimes_{D^{\prime}}\left(B \hat{\otimes}_{K} D^{\prime}\right)\right)\right)$ and similarly

$$
\mathcal{F} \otimes_{D}\left(R \hat{\otimes}_{K} D\right) \cong R \otimes_{B}\left(\left(\mathcal{F} \otimes_{D}\left(B \hat{\otimes}_{K} D\right)\right)\right) .
$$

Thus, we may assume without loss of generality that $R=B=K[[y]]$, and we need only show that $\mathcal{F}^{\prime} \otimes_{D^{\prime}}\left(D^{\prime}[[y]]\right)$ is flat over $\mathcal{F} \otimes_{D}(D[[y]])$. By $(7.38 \mathrm{~b})$ we can choose $c \in D-\{0\}$ such that $D[[y]]_{c}$ is regular and $c^{\prime} \in D^{\prime}-\{0\}$ such that $D^{\prime}[[y]]_{c^{\prime}}$ is regular. Then $d=c c^{\prime}$ has a power in $D$, and so we have $d \in D$ such that $D[[y]]_{d}$ and $D^{\prime}[[y]]_{d}$ are both regular. Since $D[[y]]_{d} \rightarrow D^{\prime}[[y]]_{d}$ is a purely inseparable extension of regular rings, the map is flat, and this is preserved by further localization.

(d) Since the composite map $S \rightarrow R \otimes S \rightarrow(R \otimes S)_{c \otimes d}$ is flat, so is $S_{d} \rightarrow$ $(R \otimes S)_{c \otimes d}$. Since $S_{d}$ is regular, it suffices to prove that the fibers of the map $S \rightarrow(R \otimes S)_{c}$ are regular. We are then free to replace $S$ by its quotient $D=S / P$ by a typical prime ideal $P$, and we must show that the generic fiber of the map $D \rightarrow(R \otimes D)_{c}$ is regular: if $D$ has fraction field $\mathcal{F}$ this means that we must show that $\mathcal{F} \otimes_{D}(R \otimes D)_{c}$ is regular (where all tensor products marked with a subscript are ordinary tensor products). If $D^{\prime}$ is a domain module-finite over $D$ with fraction field $\mathcal{F}^{\prime}$ then $\mathcal{F}^{\prime} \otimes_{D^{\prime}}\left(\left(R \otimes D^{\prime}\right)_{c}\right) \cong \mathcal{F}^{\prime} \otimes_{\mathcal{F}}\left(\mathcal{F} \otimes_{D}\left((R \otimes D)_{c}\right)\right)$ is faithfully flat over $\mathcal{F} \otimes_{D}\left((R \otimes D)_{c}\right)$. Thus, there is no loss of generality in assuming that 
$D$ is the integral closure of the regular ring $A=L\left[\left[x_{1}, \ldots, x_{n}\right]\right]$ (respectively, $\left.L\left[x_{1}, \ldots, x_{n}\right]\right)$ in a finite separable field extension of a finite purely inseparable extension of the fraction field of $A$. We give the rest of the proof only for the complete local case: the modifications needed for the case of algebras of finite type over a field are completely straightforward.

The separable part of the extension will not affect regularity. This means that we may assume without loss of generality that $D$ lies between $L[[x]]$ and $L^{1 / q}\left[\left[x^{1 / q}\right]\right]=D^{\prime}$. Let $\mathcal{F}^{\prime}$ be the fraction field of $D^{\prime}$. Then $\mathcal{F}^{\prime} \otimes_{D^{\prime}}\left(R \hat{\otimes} D^{\prime}\right)$ is faithfully flat over $\mathcal{F} \otimes_{D}(R \hat{\otimes} D)$ by part (c), and so it will suffice to prove that $\left(\mathcal{F}^{\prime} \otimes_{D^{\prime}}\left(R \hat{\otimes} D^{\prime}\right)\right)_{c}$ is regular. We have reduced to the case where $D=$ $L^{1 / q}\left[\left[x^{1 / q}\right]\right]$, and we may simplify notation and write $D=L[[x]]$. Thus, what we must show is that the generic fiber of the map $L[[x]] \rightarrow L[[R]][[x]]_{c}$ is regular, and it will suffice to show that $L[[R]][[x]]_{c}$ itself is regular. It makes the problem harder to enlarge $L$, and so we may assume that $L=L^{\infty}$ and hence that $L \supseteq K^{\infty}$. We already know that $K^{\infty}[[R]]_{c}$ is regular, and it follows that this remains true when we enlarge the field $K^{\infty}$ to $L$, by $(7.38 \mathrm{c})$. Replacing $R$ by $L[[R]]$, we see that if $R_{c}$ is regular then so is $R[[x]]_{c}$, and this follows from the fact (7.38b) that the fibers of the map $R \rightarrow R[[x]]$ are regular.

Before establishing our main results, Theorem 7.42 and Theorem 7.45 , on products, we need:

(7.41) Lemma. Let $\left(R, m, K^{\prime}\right),(S, n, L)$ be complete local rings containing a field $K$ such that $\left[K^{\prime}: K\right]$ is finite. Let $I, J$ be finitely generated ideals of $R$ and $S$ respectively and let $\otimes$ denote $\hat{\otimes}_{K}$ (respectively, $\otimes_{K}$ ). Let $M$ be a finitely generated $R$-module and $M^{\prime}$ a finitely generated $S$-module (respectively, arbitrary $R$ - and $S$-modules). Then:

(a) $\mathrm{Ann}_{M \otimes M^{\prime}} I \otimes J \cong\left(\mathrm{Ann}_{M} I\right) \otimes M^{\prime}+M \otimes\left(\operatorname{Ann}_{M^{\prime}} J\right)$.

(b) $\mathrm{Ann}_{M \otimes M^{\prime}}(I \otimes S+R \otimes J) \cong\left(\mathrm{Ann}_{M} I\right) \otimes\left(\mathrm{Ann}_{M^{\prime}} J\right)$.

Proof. (a) Let $\alpha: R \rightarrow R^{s}$ and $\beta: S \rightarrow S^{t}$ be maps such that the entries of the matrices are sets of generators for $I, J$, respectively. The entries of a matrix for $\alpha \otimes \beta$ generate $I \otimes J$ and

$$
\operatorname{Ann}_{M \otimes M^{\prime}} I \otimes J=\operatorname{Ker}\left(\left(M \otimes M^{\prime}\right) \otimes_{R \otimes S}(\alpha \otimes \beta)\right) \cong\left(M \otimes_{R} \alpha\right) \otimes\left(M^{\prime} \otimes_{S} \beta\right) .
$$

$\operatorname{Ker}\left(M \otimes_{R} \alpha\right)=\operatorname{Ann}_{M} I$ and so $M \otimes_{R} \alpha$ induces an injection $\left(M / \operatorname{Ann}_{M} I\right) \rightarrow$ $M^{s}$; similarly, $M^{\prime} \otimes_{S} \beta$ induces an injection $\left(M^{\prime} / \mathrm{Ann}_{M^{\prime}} J\right) \rightarrow M^{\prime t}$. Since $\otimes$ is exact, we have an induced injection of $\left(M / \operatorname{Ann}_{M} I\right) \otimes\left(M^{\prime} / \operatorname{Ann}_{M^{\prime}} J\right) \cong$ $\left(M \otimes M^{\prime}\right) /\left(M \otimes \mathrm{Ann}_{M^{\prime}} J+\mathrm{Ann}_{M} I \otimes M^{\prime}\right)$ into $\left(M \otimes M^{\prime}\right)^{s t}$ such that the corresponding map $M \otimes M^{\prime} \rightarrow\left(M \otimes M^{\prime}\right)^{s t}$ is isomorphic to $\left(M \otimes M^{\prime}\right) \otimes_{R \otimes S}(\alpha \otimes \beta)$. The result now follows.

(b) $\operatorname{Ann}_{M} I=\operatorname{Ker}(M \otimes \alpha)$ (with $\alpha$ as in the proof of (a)); applying $\otimes M^{\prime}$ yields that $\left(\mathrm{Ann}_{M} I\right) \otimes M^{\prime}=\mathrm{Ann}_{M \otimes M^{\prime}} I \otimes S$. Similarly, $M \otimes \mathrm{Ann}_{M^{\prime}} I^{\prime}=$ $\mathrm{Ann}_{M \otimes M^{\prime}} R \otimes J$. Thus, $\mathrm{Ann}_{M \otimes M^{\prime}}(I \otimes S+R \otimes J)=\left(\left(\operatorname{Ann}_{M} I\right) \otimes M^{\prime}\right) \cap(M \otimes$ $\left.\left(\mathrm{Ann}_{M^{\prime}} J\right)\right)=\operatorname{Ann}_{M} I \otimes \mathrm{Ann}_{M^{\prime}} J$.

(7.42) Theorem. Let $(R, m, K),(S, n, L)$ be complete local rings with coefficient fields $K, L$ (respectively, finitely generated algebras over fields $K, L$ ) where $K, L$ have characteristic $p$ and $K \subseteq L$. Suppose that $K^{1 / q}\left[\left[R_{\mathrm{red}}\right]\right]$ is 
reduced for all $q$ (respectively, that the total quotient ring of $R_{\mathrm{red}}$ is separable over $K)$. Then:

(a) There are weak test elements $c \in R^{o}$ and $d \in S^{o}$ such that $c \otimes d$ (in the complete case we use this notation for $c \hat{\otimes} d)$ is a weak test element in $R \hat{\otimes}_{K} S$ (respectively, $R \otimes_{K} S$ ). If $R, S$ are reduced then the word "weak" can be omitted.

(b) Let $N \subseteq M$ be finitely generated $R$-modules, and let $N^{\prime} \subseteq M^{\prime}$ be finitely generated $S$-modules. Suppose that $N$ is tightly closed in $M$ and $N^{\prime}$ is tightly closed in $M^{\prime}$. Suppose either that

(i) $K$ is perfect or

(ii) $M / N$ and $M^{\prime} / N^{\prime}$ are finite length modules with socles of length one (in the case of finitely generated algebras, if the socle in $M / N$ is $\cong R / m=K^{\prime}$ and the socle in $M^{\prime} / N^{\prime} \cong S / n=L^{\prime}$, also assume that $K^{\prime} \otimes_{K} L^{\prime}$ is a field).

Then $N \hat{\otimes}_{K} M^{\prime}+M \hat{\otimes}_{K} N^{\prime}$ ( respectively, $N \otimes_{K} M^{\prime}+M \otimes_{K} N^{\prime}$ ) is tightly closed in $M \hat{\otimes}_{K} M^{\prime}$ (respectively, in $M \otimes_{K} M^{\prime}$ ).

Proof. Except for a few parenthetical comments, we shall give the proof only for the complete local case and complete tensor products: the argument for algebras of finite type and ordinary tensor product is entirely similar.

(a) It suffices to do the case where $R, S$ are reduced. Since $S$ is reduced and the singular locus is closed we can choose $d \in S^{o}$ such that $S_{d}$ is regular. By $(7.40 \mathrm{~b})$ we can choose $c \in R^{o}$ such that $K^{1 / q}[[R]]_{c}$ is geometrically regular over $K^{1 / q}$ for all $q$. By $(7.40 \mathrm{~d})$ we have that $\left(R \hat{\otimes}_{K} S\right)_{c \otimes d}$ is regular. Since $\hat{\otimes}_{K}$ is exact, $c \otimes 1$ and $1 \otimes d$ are nonzerodivisors in $R \hat{\otimes}_{K} S$, and hence so is $c \otimes d$. By Theorem 6.2, $c \otimes d$ has a power which is a (completely stable) test element for $R \hat{\otimes}_{K} S$.

(b) First off, we can replace $M, M^{\prime}$ by $M / N$ and $M^{\prime} / N^{\prime}$. The assertion becomes that if 0 is tightly closed in $M$ and 0 is tightly closed in $M^{\prime}$ then 0 is tightly closed in $M \hat{\otimes}_{K} M^{\prime}$. In case (i), where the modules are not assumed to have finite length, we can nonetheless reduce to that case, since, in each module, 0 will be an intersection of tightly closed submodules whose quotients are finite length. Since both $R, S$ have weak test elements, by [HH4], (8.13b) we may assume without loss of generality that each of $M, M^{\prime}$ is killed by a power of a maximal ideal. (This reduction will also work in the affine case: the residue fields $K^{\prime}, L^{\prime}$ at the appropriate maximal ideals of $R$ and $S$ respectively will be finite algebraic extensions of $K$ and $L$. Note that the notions of length and dimension over $K$ or $L$ will be different.) We need to show that if either (i) $K$ is perfect or (ii) the socles in $M, M^{\prime}$ are one-dimensional (and $K^{\prime} \otimes_{K} L^{\prime}$ is a field), then 0 is tightly closed in $M \otimes_{K} M^{\prime}$ : note that since $M, M^{\prime}$ have finite length, we may write $M \otimes_{K} M^{\prime}$ instead of $M \hat{\otimes}_{K} M^{\prime}$.

Let $T=R \hat{\otimes}_{K} S$. It is easy to see that $F_{T}^{e}\left(M \hat{\otimes}_{K} M^{\prime}\right) \cong F_{R}^{e}(M) \hat{\otimes}_{F_{K}^{e}(K)} F_{S}^{e}\left(M^{\prime}\right)$. Here, we might have written " $K$ " instead of " $F_{K}^{e}(K)$ ": we have used the latter notation to make it clear how $K$ is acting on $F_{R}^{e}(M)$ and $F_{S}^{e}\left(M^{\prime}\right)$.

If the socles in $M, M^{\prime}$ have length one and are generated by $u, u^{\prime}$ respectively, then the socle in $M \hat{\otimes}_{K} M^{\prime}$ is generated by $u \otimes u^{\prime}$, since $R u \hat{\otimes}_{K} S u^{\prime}=$ $\mathrm{Ann}_{M} m \hat{\otimes}_{K} \mathrm{Ann}_{M^{\prime}} n \cong \mathrm{Ann}_{M \hat{\otimes} M^{\prime}}(m \hat{\otimes} S+R \hat{\otimes} n)$ by (7.41b). In the complete local case, this socle has length one over $R \hat{\otimes} S$. (In the case of affine algebras, the socle still has length one because of the assumption that $K^{\prime} \otimes_{K} L^{\prime}$ 
is a field.) Thus, if 0 is not tightly closed in $M \hat{\otimes}_{K} M^{\prime}$, then $u \otimes u^{\prime}$ is in the tight closure of 0 . But then $(c \otimes d)\left(u \otimes u^{\prime}\right)^{q}=\left(c u^{q}\right) \otimes\left(d u^{\prime q}\right)=0$ in $F_{T}^{e}\left(M \hat{\otimes}_{K} M^{\prime}\right) \cong F_{R}^{e}(M) \hat{\otimes}_{F_{K}^{e}(K)} F_{S}^{e}\left(M^{\prime}\right)$ for all $q \gg 0$, and this implies that $c u^{q}=0$ for infinitely many $q$ or else that $d u^{\prime q}=0$ for infinitely many $q$. By [HH4], Lemma 8.16, if a ring has a weak test element, $c^{\prime}$ is not in any minimal prime, and $c^{\prime} x^{q}=0$ (or is in $0^{*}$ ) for infinitely many values of $q$, then $x$ is in the tight closure of 0 . Thus, either $u \in 0_{M}^{*}$ or $u^{\prime} \in 0_{M^{\prime}}^{*}$, a contradiction.

Now suppose that $K$ is perfect. Let $c \in R^{o}$ and $d \in S^{o}$ be as in part (a), so that $c$ is a weak test element in $R, d$ is a weak test element in $S$, and $c \otimes d$ is a weak test element in $T=R \otimes S$. Let $V_{e}$ denote the set of all elements $v$ in $M$ such that $c v^{q}=0$ (where $q=p^{e}$ ) and let $W_{e}$ be the set of all elements $w$ in $M^{\prime}$ such that $d w^{q}=0$. Then the set $Y_{e}$ of all elements $y \in M \otimes M^{\prime}$ such that $(c \otimes d) y^{q}=0$ may be identified with $V_{e} \otimes M^{\prime}+M \otimes W_{e}$. Since $K$ is perfect we may identify $F_{R}^{e}(M) \otimes_{F^{e}(K)} F_{S}^{e}\left(M^{\prime}\right)$ with $F_{R}^{e}(M) \otimes_{K} F_{S}^{e}\left(M^{\prime}\right)$. The kernel of multiplication by $c \otimes d$ is $X_{e}=\left(\operatorname{Ann}_{F_{R}^{e}(M)} c\right) \otimes M^{\prime}+M \otimes\left(\operatorname{Ann}_{F_{S}^{e}\left(M^{\prime}\right)} d\right)$ by (7.41a). Thus, $Y_{e}$ is the inverse image of $X_{e}$ under the map $M \otimes_{K} N \rightarrow$ $F_{R}^{e}(M) \otimes_{K} F_{S}^{e}\left(M^{\prime}\right)$, where the two copies of $K$ over which we are tensoring may be thought of as the same (if $K$ were not perfect, we would have to write $F_{K}^{e}(K)$ for the second). Now, quite generally, given maps of $K$-vector spaces $\alpha: M \rightarrow H, \beta: M^{\prime} \rightarrow H^{\prime}$, the inverse image under $\alpha \otimes_{K} \beta$ of a subspace of the form $H_{0} \otimes_{K} H^{\prime}+H \otimes_{K} H_{0}^{\prime} \subseteq H \otimes_{K} H^{\prime}$ is $\alpha^{-1}\left(H_{0}\right) \otimes_{K} M^{\prime}+M \otimes \beta^{-1}\left(H_{0}^{\prime}\right)$ : this follows from the injectivity of the tensor product over $K$ of the injective maps $M / \alpha^{-1}\left(H_{0}\right) \rightarrow H / H_{0}$ and $M^{\prime} / \beta^{-1}\left(H_{0}^{\prime}\right) \rightarrow H^{\prime} / H_{0}^{\prime}$. The statement that $Y_{e}$ may be identified with $V_{e} \otimes_{K} M^{\prime}+M \otimes_{K} W_{e}$ now follows.

Let us suppose that $c, d$, and $c \otimes d$ are all $q^{\prime}$-weak test elements, where $q^{\prime}=p^{e^{\prime}}$. If 0 is not tightly closed in $M \otimes_{K} N$ we see that there is a nonzero element in $\bigcap_{e>e^{\prime}}\left(V_{e} \otimes_{K} M^{\prime}+M \otimes_{K} W_{e}\right)$ in $M \otimes_{K} M^{\prime}$. The result is now immediate because from part (c) of Proposition 7.9 we know that $V_{e}=0$ and $W_{e}=0$ for $e \gg 0$.

(7.43) Example. We can obtain an instructive example by modifying Example 6.18. Let $K$ be a field containing an element $t$ that is not a $p$ th power, let $L=K[\theta]$ where $\theta=t^{1 / p}$, let $R=K\left[\left[x, y, z_{1}, \ldots, z_{n}\right]\right] /(f)$ where $f=$ $x^{p}-t y^{p}-\sum_{j=1}^{n} z_{j}^{h_{j}}$ where every $h_{j}$ is an integer strictly bigger than $p$ and not divisible by $p$ (for definiteness, we may take all the $h_{j}$ to be $p+1$ ). Let $S=K[[u, \theta u]] \subseteq L[[u]]$, where $u$ is a new indeterminate. Then the prime ideal $P=\left(z_{j}\right) R$ is tightly closed in $S$, but $P \hat{\otimes}_{K} S$ is not tightly closed in $R \hat{\otimes}_{K} S: u x-u \theta y$ is in the tight closure but not in the ideal (its $p$ th power is $\left.u^{p}\left(x^{p}-t y^{p}\right) \in\left(z_{j}^{h_{j}}\right) \subseteq\left(z_{j}^{p}\right)\right)$.

(7.44) Definitions and discussion. Let $R$ be a Noetherian $K$-algebra where $K$ is a field of characteristic $p$. We say that $R$ is geometrically weakly $F$ regular (respectively, geometrically $F$-regular) over $K$ if for every finite purely inseparable extension $K^{\prime}$ of $K, K^{\prime} \otimes_{K} R$ is weakly $F$-regular (respectively, $F$-regular) over $K$. Note that if $R$ is geometrically weakly $F$-regular over $K$ then, in particular, for every $K$-subalgebra $T$ of $R$, if $K^{\prime}$ is a finite purely inseparable extension of $K, K^{\prime} \otimes_{K} T \subseteq K^{\prime} \otimes_{K} R$ is reduced. Thus if $T=L \subseteq R$ is a field, then $L$ is separable over $K$ and $L\left[K^{\infty}\right] \subseteq L^{\infty}$ may be identified with $L \otimes_{K} K^{\infty}$. 
If $R$ is (essentially) of finite type over a field $L$ containing $K$ then $K^{\infty} \otimes_{K}$ $R \cong K^{\infty} \otimes_{K}\left(L \otimes_{L} S\right) \cong\left(L\left[K^{\infty}\right]\right) \otimes_{L} R$ if $L$ is separable over $K$. Thus, $R$ is geometrically (weakly) $F$-regular iff $K^{\infty} \otimes_{K} R$ is (weakly) $F$-regular, since the latter is faithfully flat over $K^{\prime} \otimes_{K} R$ for $K^{\prime} \subseteq K^{\infty}$ finite over $K$ and is the direct limit of these in a system with faithfully flat maps (cf. (6.14a)). Notice that it is possible that $L\left[K^{\infty}\right] \otimes_{L} R$ is weakly $F$-regular even though $L$ is not separable over $K$ : this occurs, for example when $R=L$.

(7.45) Theorem. Let $R$ and $S$ be rings of characteristic $p$.

(a) Let $(R, m, K)$ and $(S, n, L)$ be complete local rings with coefficient fields $K$ and $L$ and suppose that $R$ and $S$ are weakly $F$-regular. Then $R \hat{\otimes}_{K} S$ is weakly $F$-regular.

(b) Let $R, S$ be finitely generated algebras over fields $K$ and $L$ with $K \subseteq$ $L$.

(i) Suppose that $R_{m}, S_{n}$ are weakly $F$-regular for maximal ideals $m, n$ such that $(R / m) \otimes_{K}(S / n)$ is a field. Then $\left(R \otimes_{K} S\right)_{Q}$ is weakly $F$-regular, where $Q$ is the maximal ideal $m \otimes_{K} S+R \otimes_{K} n$.

(ii) Suppose that $R$ is geometrically weakly $F$-regular over $K$, and that $S$ is geometrically weakly $F$-regular over $K$ (respectively, that $L\left[K^{\infty}\right] \otimes_{R} S$ is weakly $F$-regular over $K$ ). Then $R \otimes_{K} S$ is geometrically weakly $F$-regular over $K$ (respectively, weakly $F$ regular over $K$ ).

Proof. (a) Because $R, S$ are weakly $F$-regular, they are normal and, hence, approximately Gorenstein (cf. [Ho4] and [HH4], (8.6)). Thus, there are decreasing sequences $\left\{I_{t}\right\}_{t},\left\{J_{t}\right\}_{t}$, respectively of $m$ - (respectively, $n$-) primary irreducible ideals cofinal with the powers of $m$ (respectively, $n$ ) in $R$ (respectively, $S$ ). Then $\left(R / I_{t}\right) \hat{\otimes}_{K}\left(S / J_{t}\right)$ has a one-dimensional socle (cf. the proof of (7.42bii)) and so $\left\{I_{t} \hat{\otimes}_{K} S+R \hat{\otimes}_{K} J_{t}\right\}_{t}$ is a sequence of irreducible ideals in $R \hat{\otimes}_{K} S$ primary to the maximal ideal $Q$ and cofinal with the powers of $Q$. Thus, it suffices to check that $I_{t} \hat{\otimes}_{K} S+R \hat{\otimes}_{K} J_{t}$ is tightly closed for every $t$, which is immediate from $(7.42 \mathrm{bii})$.

The proof of (bi) is virtually identical to the proof of (a): simply use the affine case of (7.42bii).

To prove (bii), first note that the discussion in (7.44) permits us to reduce to the case where $K$ is perfect (replacing $K, L, R, S$ by $K^{\infty}, L\left[K^{\infty}\right]$, $K^{\infty} \otimes_{K} R$, and $L\left[K^{\infty}\right] \otimes_{L} S \cong K^{\infty} \otimes_{K} S$, respectively: $K^{\infty} \otimes\left(R \otimes_{K} S\right) \cong$ $\left(K^{\infty} \otimes_{K} R\right) \otimes_{K^{\infty}}\left(L\left[K^{\infty}\right] \otimes_{L} S\right)$ ). (In the parenthetical case we do not have this last isomorphism, but it is true that $\left(K^{\infty} \otimes_{K} R\right) \otimes_{K^{\infty}}\left(L\left[K^{\infty}\right] \otimes_{L} S\right) \cong$ $L\left[K^{\infty}\right] \otimes_{L}\left(R \otimes_{K} S\right)$ is faithfully flat over $R \otimes_{K} S$, which permits the reduction.) Thus, we henceforth assume that $K$ is perfect.

Let $Q$ be an arbitrary maximal ideal of $R \otimes_{K} S$, and suppose it lies over the (necessarily maximal) ideal $m \subseteq R$. It will suffice to show that $\left(R \otimes_{K} S\right)_{Q}$ is weakly $F$-regular. Let $\Omega$ be a finite algebraic extension field of $R / m$ which is normal over $K$ : since $K$ is perfect, $\Omega$ is a finite Galois extension of $K$. Since $K \rightarrow \Omega$ is smooth, so are $R \rightarrow \Omega \otimes_{K} R$, and $S \rightarrow \Omega \otimes_{K} S \cong\left(\Omega \otimes_{K} L\right) \otimes_{L} S$, and it follows from (7.25d) that $\Omega \otimes_{K} R$ and $\Omega \otimes_{K} S$ are weakly $F$-regular. Choose a maximal ideal $Q^{\prime}$ of $\Omega \otimes_{K}\left(R \otimes_{K} S\right)$ lying over $Q$. It will suffice to show that $\left.\Omega \otimes_{K}\left(R \otimes_{K} S\right)\right)_{Q^{\prime}}$, which is faithfully flat over $\left(R \otimes_{K} S\right)_{Q}$, is weakly $F$-regular. $Q^{\prime}$ lies over a maximal ideal $m^{\prime}$ of $\Omega \otimes_{K} R$ which in turn 
lies over $m$ in $R$. Thus, $m^{\prime}$ corresponds to a maximal ideal of $\Omega \otimes_{K}(R / m)$. Since $\Omega$ is a normal extension of $K$ containing $R / m$, each such maximal ideal corresponds to a $K$-embedding of $R / m$ into $\Omega$, and has $\Omega$ as its residue field. Thus, $\left(\Omega \otimes_{K} R\right) / m^{\prime} \cong \Omega$.

Now $\Omega \otimes_{K} L$ is a finite product, $\prod_{j} L_{j}$, of fields $L_{j} \supseteq \Omega$, and $\Omega \otimes_{K}\left(R \otimes_{K}\right.$ $S) \cong\left(\Omega \otimes_{K} L\right) \otimes_{L}\left(R \otimes_{K} S\right)$ has a corresponding decomposition as $\prod_{j} L_{j} \otimes_{L}$ $\left(R \otimes_{K} S\right)$. We may choose $j$ so that the point $Q^{\prime}$ corresponds to a point $Q^{\prime \prime}$ of $\operatorname{Spec}\left(L_{j} \otimes_{L}\left(R \otimes_{K} S\right)\right)$. We may then replace $K$ by $\Omega, L$ by the field $L_{j} \supseteq \Omega$ chosen just above, $R$ by $\Omega \otimes_{K} R$, and $S$ by $L_{j} \otimes_{L} S$. Note that $L_{j} \otimes_{K}\left(R \otimes_{K} S\right) \cong$ $\left(\Omega \otimes_{K} R\right) \otimes_{\Omega}\left(L_{j} \otimes_{L} S\right)$, and so $\left(\Omega \otimes_{K}\left(R \otimes_{K} S\right)\right)_{Q^{\prime}} \cong\left(L_{j} \otimes_{K}\left(R \otimes_{K} S\right)\right)_{Q^{\prime \prime}} \cong$ $\left(\left(\Omega \otimes_{K} R\right) \otimes_{\Omega}\left(L_{j} \otimes_{L} S\right)\right)_{Q^{\prime \prime}}$.

Changing back to our original notation, we see that we may assume without loss of generality that $R / m=K$. But then the maximal ideals of $R \otimes_{K} S$ lying over $m$ simply correspond to the maximal ideals $n$ of $S$, and we have $Q=m \otimes_{K} S+R \otimes n$. We are now in the situation of (bi), and the proof is complete.

(7.46) Corollary. If $R$ is a finitely generated algebra over a field $K$ of characteristic $p$ and $R$ is geometrically weakly $F$-regular over $K$ then $\Omega \otimes_{K} R$ is geometrically weakly $F$-regular over $\Omega$ for every field $\Omega \supseteq K$.

Proof. It suffices to prove that $\Omega^{\infty} \otimes_{K} R$ is weakly $F$-regular. Since $R$ is geometrically weakly $F$-regular, and $\Omega^{\infty}\left[K^{\infty}\right]=\Omega^{\infty}$ is weakly $F$-regular, this is immediate from the parenthetical version of (7.45bii), taking $S=L=\Omega^{\infty}$.

(7.47) Remark. In (7.43bii), one does need the geometric weak $F$-regularity of $R$ to conclude that $R \otimes_{K} S$ is weakly $F$-regular: $S$ might be $L\left[K^{\prime}\right]$ with $K^{\prime} \subseteq K^{\infty}$. On the other hand, once this is assumed, it should not be necessary to assume that $L\left[K^{\infty}\right] \otimes_{K} S$ is weakly $F$-regular to insure that $R \otimes_{K} S$ is weakly $F$-regular: the weak $F$-regularity of $S$ itself should suffice. (One cannot take, for example, $R=K^{\prime} \subseteq K^{\infty}$, since this is not geometrically weakly $F$-regular over $K$.) While we have not been able to prove a stronger result, one expects weak $F$-regularity to behave quite similarly to regularity in the way that it is or is not preserved in products and under base extensions. This is one reason for thinking that a stronger result should hold. If $R$ is geometrically regular over $K$ and $S$ is weakly $F$-regular then one does, in fact, have the stronger result, since $S \rightarrow R \otimes_{K} S$ has a weakly $F$-regular base and regular fibers and we may apply Theorem $7.25 \mathrm{~d}$.

\section{Weakly $F$-REgUlar AFFINE $K$-ALgebras ARE $F$-REgUlar OVER "BIG" FIELDS $K$.}

The proof of the result below in the case where the field is uncountable was shown to us by M. P. Murthy, who also suggested that the version given here should be true.

(8.1) Theorem. Let $K$ be a field of characteristic $p$ of infinite transcendence degree over its prime field and let $R$ be a finitely generated $K$-algebra. Suppose that $R$ is weakly $F$-regular. Then $R$ is $F$-regular.

Proof. Since $R$ is weakly $F$-regular it is a normal ring, and, hence, a finite product of domains. It suffices to consider each factor separately. Thus, we 
may suppose without loss of generality that $R$ is a domain.

We first treat the case where the field $K$ is uncountable, utilizing an argument of M. P. Murthy. We want to show that for every multiplicative system $W$ of $R$, the ring $W^{-1} R$ is weakly $F$-regular. Since every ideal of this ring is expanded from $R$, if the condition fails there is an ideal $I$ of $R$ such that $I W^{-1} R$ is not tightly closed in $W^{-1} R$. Choose an element $u$ of the tight closure not in the ideal. After multiplying by an element of $W$, we may assume that $u \in R$. It follows that there is an element $c \in R^{o}$ such that for all nonnegative integers $e$ there is an element $f_{e} \in W$ such that $c f_{e} u^{q} \in I^{[q]}$. We see at once that if we replace $W$ by the multiplicative system generated by all the $f_{e}$, we obtain a new counterexample in which $W$ is countable. Thus, there is no loss of generality in assuming that $W$ is countable. Moreover, we may choose a maximal ideal $m$ of the ring $S=W^{-1} R$ such that the image of $u$ in $S_{m}$ is not in $I S_{m}$, although it evidently is still in the tight closure of $I S_{m}$. It follows that $S_{m}$ is not weakly $F$-regular. But the contraction $P$ of $m$ to $R$ must be a maximal ideal of $R$. (This is well known: the point is that if the domain $\mathrm{R} / \mathrm{P}$ is not a field, it cannot become a field when one localizes at a countable multiplicative system if $K$ is uncountable. To see this, write it as a module-finite extension of a polynomial ring, replace each element of the multiplicative system by a nonzero multiple in the polynomial ring, and use the fact that a polynomial ring in one or more variables over an uncountable field contains uncountably many irreducible elements that are not associates.) But $S_{m} \cong R_{P}$, and if $P$ is maximal, $R_{P}$ is weakly $F$-regular by Theorem $3.4 \mathrm{~b}$.

We next treat the case where $K$ is only assumed to have infinite transcendence degree over its prime field. Let $\Lambda$ be an uncountable index set and let $\left\{z_{\lambda}: \lambda \in \Lambda\right\}$ be a family of algebraically independent indeterminates over $R$ indexed by $\Lambda$. We write $K(z)$ for the purely transcendental extension of $K$ obtained by adjoining all the $z_{\lambda}$. Choose a nonzero element $c \in R$ such that $R_{c}$ is regular. Let $T=K(z) \otimes_{K} R$, so that $T_{c}$ may be identified with $K(z) \otimes_{K} R_{c}$. Then $T_{c}$ is regular (when the set $\Lambda$ is finite we have a localization of a polynomial ring in finitely many variables over $R_{c}$, and the general case then follows easily by a direct limit argument). By replacing $c$ by a suitable power we may assume that $c$ is a test element for $T$. To complete the proof, it will suffice to show that $T$ is weakly $F$-regular, for then it follows from the first part of the proof (since $K(z)$ is uncountable) that $T$ is $F$-regular, and since $T$ is faithfully flat over $R, R$ will be $F$-regular as well.

Next, we may choose a subfield $L$ of $K$ finitely generated over the prime field of $K$ containing all the coefficients of the polynomials occurring in a finite presentation for $R$ over $K$. We may therefore write $R=K \otimes_{L} R_{0}$, where $R_{0}$ is a finitely generated $L$-algebra. It follows that $R_{0}$ may be identified with a subring of $R$ and is also a domain. Moreover, by enlarging $L$, if necessary, we may suppose that $c \in R_{0}$. Suppose that $T$ is not weakly $F$-regular. Then we can choose finitely many elements $f_{1}, \ldots, f_{n}$ and an element $u$ of $T$ such that $u$ is in the tight closure of the ideal $I$ generated by the $f_{i}$ but not in $I$. We shall obtain a contradiction. After enlarging $L$, if necessary, we may choose finitely many of the $z_{\lambda}$, say $z_{1}, \ldots, z_{h}$, such that $u$ and $f_{1}, \ldots, f_{n}$ are in $L\left(z_{1}, \ldots, z_{h}\right) \otimes_{L} R_{0}=R_{1}$. Since $c$ is a test element for $T$, we have that $c u^{q} \in\left(f_{1}^{q}, \ldots, f_{n}^{q}\right) T$ for all nonnegative integers $e$. Since $T$ is faithfully flat over $R_{1}$ and all of the elements $c, u, f_{i}$ are in $R_{1}$, it follows 
that $c u^{q} \in\left(f_{1}^{q}, \ldots, f_{n}^{q}\right) R_{1}$ for all $q$, and so $u$ is in the tight closure of $I_{1}=$ $\left(f_{1}, \ldots, f_{n}\right) R_{1}$ in $R_{1}$, but not in $I_{1}$, since it is not even in $I_{1} T$. It follows that $R_{1}$ is not weakly $F$-regular. However, we may choose $y_{1}, \ldots, y_{n}$ in $K$ algebraically independent over $L$, and the $L$-isomorphism of $L\left(z_{1}, \ldots, z_{h}\right) \cong$ $L\left(y_{1}, \ldots, y_{h}\right)=L(y)$ that sends $z_{i}$ to $y_{i}$ for every $i$ induces an isomorphism of $R_{1}$ with $R_{2}=L(y) \otimes_{L} R_{0}$. It follows that $R_{2}$ is not weakly $F$-regular. But since $L \subseteq L(y) \subseteq K$, we have that $R \cong K \otimes_{L(y)} R_{2}$, by the associativity of $\otimes$, and since $R$ is $\mathrm{f}$ aithfully flat over $R_{2}$ and weakly $F$-regular, it follows that $R_{2}$ is weakly $F$-regular, a contradiction.

\section{BIBLIOGRAPHY}

[Ab1] I. Aberbach, Finite phantom projective dimension, Amer. J. Math. 116 (1994), 447-477.

[Ab2] _ Test elements in excellent rings with an application to the uniform Artin-Rees property, Proc. Amer. Math. Soc. 118 (1993), 355-363.

[Ab3] - Tight closure in F-rational rings, Nagoya Math. J. (to appear).

[AHH] I. Aberbach, M. Hochster, and C. Huneke, Localization of tight closure and modules of finite phantom projective dimension, J. Reine Angew. Math. 434 (1993), 67-114.

[B] J.-F. Boutot, Singularités rationelles et quotients par les groupes réductifs, Invent. Math. 88 (1987), 65-68.

[BrS] J. Briançon and $\mathrm{H}$. Skoda, Sur la clôture intégrale d'un idéal de germes de fonctions holomorphes en un point de $C^{n}$, C. R. Acad. Sci. Paris Sér. I. Math. 278 (1974), 949-951.

[Du] S. P. Dutta, On the canonical element conjecture, Trans. Amer. Math. Soc. 299 (1987), 803-811.

[EGA] A. Grothendieck (rédigés avec la collaboration de J. Dieudonné), Éléments de géométrie algébrique IV: Étude local des schémas et des morphismes de schémas (Seconde partie), Inst. Hautes Etudes Sci. Publ. Math. 24 (1965), 1-231.

[EvG1] E. G. Evans and P. Griffith, The syzygy problem, Ann. of Math. (2) 114 (1981), 323-333.

[EvG2] _ Syzygies, London Math. Soc. Lecture Note Ser., vol. 106, Cambridge Univ. Press, London and New York, 1985.

[EvG3] _ Order ideals, Commutative Algebra, Math. Sci. Res. Inst. Publ. 15, SpringerVerlag, New York, Berlin, and Heidelberg, 1989, pp. 213-225.

[FeW] R. Fedder and K. Watanabe, $A$ characterization of $F$-regularity in terms of $F$-purity, Commutative Algebra, Math. Sci. Res. Inst. Publ. 15, Springer-Verlag, New York, Berlin, andHeidelberg, 1989, pp. 227-245.

[Gla] D. J. Glassbrenner, Invariant rings of group actions, determinantal rings, and tight closure, Thesis, Univ. of Michigan, 1992.

[GrHa] A. Grothendieck (notes by R. Hartshorne), Local cohomology, Lecture Notes in Math., vol. 41, Springer-Verlag, Heidelberg, 1967.

[Ho1] M. Hochster, Contracted ideals from integral extensions of regular rings, Nagoya Math. J. 51 (1973), 25-43.

[Ho2] _ Topics in the homological theory of modules over commutative rings, C.B.M.S. Regional Conf. Ser. in Math., no. 24, Amer. Math. Soc., Providence, RI, 1975.

[Ho3] _ Big Cohen-Macaulay modules and algebras and embeddability in rings of Witt vectors, (Proc. Queen's Univ. Commutative Algebra Conf.), Queen's Papers in Pure and Applied Math. 42 (1975), 106-195.

[Ho4] _ Cyclic purity versus purity in excellent Noetherian rings, Trans. Amer. Math. Soc. 231 (1977), 463-488.

[Ho5] - Some applications of the Frobenius in characteristic 0, Bull. Amer. Math. Soc. 84 (1978), 886-912.

[Ho6] - Cohen-Macaulay rings and modules, Proc. Internat. Congress Math., (Helsinki, Finland), Vol. I, Acad. Sci. Fennica, 1980, pp. 291-298. 
[Ho7] Canonical elements in local cohomology modules and the direct summand conjecture, J. Algebra 84 (1983), 503-553.

[Ho8] _ Solid closure, Commutative Algebra: Syzygies, Multiplicities and Birational Algebra, Contemp. Math., vol. 159, Amer. Math. Soc., Providence, RI, 1994, pp. 103-172.

[Ho9] _ Tight closure in equal characteristic, big Cohen-Macaulay algebras, and solid closure, Commutative Algebra: Syzygies, Multiplicities and Birational Algebra, Contemp. Math., vol. 159, Amer. Math. Soc., Providence, RI, 1994, pp. 173-196.

[HoE] M. Hochster and J. A. Eagon, Cohen-Macaulay rings, invariant theory, and the generic perfection of determinantal loci, Amer. J. Math. 93 (1971), 1020-1058.

[HH1] M. Hochster and C. Huneke, Tightly closed ideals, Bull. Amer. Math. Soc. 18 (1988), 45-48.

[HH2] , Tight closure, Commutative Algebra, Math. Sci. Res. Inst. Publ. 15, SpringerVerlag, New York, Berlin, Heidelberg, 1989, pp. 305-324.

[HH3] _ Tight closure and strong F-regularity, Mém. Soc. Math. France 38 (1989), 119133.

[HH4] _ Tight closure, invariant theory, and the Briançon-Skoda theorem, J. Amer. Math. Soc. 3 (1990), 31-116.

[HH5] _ Absolute integral closures are big Cohen-Macaulay algebras in characteristic $p$, Bull. Amer. Math. Soc. (N. S.) 24 (1991), 137-143.

[HH6] - Tight closure and elements of small order in integral extensions, J. Pure Appl. Algebra 71 (1991), 233-247.

[HH7] _ Infinite integral extensions and big Cohen-Macaulay algebras, Ann. of Math. (2) 135 (1992), 53-89.

[HH8] _ Phantom homology, Mem. Amer. Math. Soc. 103 (1993), 1-91.

[HH9] - Tight closures of parameter ideals and splitting in module-finite extensions, $\mathbf{J}$. Algebraic Geom. (to appear).

[HH10] - Tight closure in equal characteristic zero (in preparation).

[HH11] _ Applications of the existence of big Cohen-Macaulay algebras, Adv. Math. (to appear).

[HR1] M. Hochster and J. L. Roberts, Rings of invariants of reductive groups acting on regular rings are Cohen-Macaulay, Adv. Math. 13 (1974), 115-175.

[HR2] _ The purity of the Frobenius and local cohomology, Adv. Math. 21 (1976), 117-172.

[Hul] C. Huneke, An algebraist commuting in Berkeley, Math. Intelligencer 11 (1989), 40-52.

[Hu2] Absolute integral closures and big Cohen-Macaulay algebras, Proc. 1990 Internat Congress Math. (Kyoto, 1990), Vol. I, Math. Soc. Japan, Springer-Verlag, New York, Berlin, Heidelberg, 1991, pp. 339-349.

[Hu3] _ Uniform bounds in Noetherian rings, Invent. Math. 107 (1992), 203-223.

[Ke] G. Kempf, The Hochster-Roberts theorem of invariant theory, Michigan Math. J. 26 (1979), 19-32.

[Ku1] E. Kunz, Characterizations of regular local rings of characteristic p , Amer. J. Math. 91 (1969), 772-784.

[Ku2] - On Noetherian rings of characteristic p, Amer. J. Math. 98 (1976), 999-1013.

[LS] J. Lipman and A. Sathaye, Jacobian ideals and a theorem of Briançon-Skoda, Michigan Math. J. 28 (1981), 199-222.

[LT] J. Lipman and B. Teissier, Pseudo-rational local rings and a theorem of Briançon-Skoda about integral closures of ideals, Michigan Math. J. 28 (1981), 97-116.

[Ma] F. Ma, Splitting in integral extensions, Cohen-Macaulay modules and algebras, J. Algebra 116 (1988), 176-195.

[Mat] H. Matsumura, Commutative algebra, Benjamin, 1970.

[N] M. Nagata, Local rings, Interscience, New York, 1972.

[PS1] C. Peskine and L. Szpiro, Dimension projective finie et cohomologie locale, Inst. Hautes Etudes Sci. Publ. Math. 42 (1973), 323-395. 
[PS2] _ Syzygies et multiplicités, C. R. Acad. Sci. Paris Sér. A Math. 278 (1974), 14211424.

[Ro1] P. Roberts, Two applications of dualizing complexes over local rings, Ann. Sci. Ecole. Norm. Sup. 9 (1976), 103-106.

[Ro2] Cohen-Macaulay complexes and an analytic proof of the new intersection conjecture, J. Algebra 66 (1980), 225-230.

[Ro3] _ The vanishing of intersection multiplicities of perfect complexes, Bull. Amer. Math. Soc. 13 (1985), 127-130.

[Ro4] L Le théorème d'intersection, C. R. Acad. Sci. Paris Sér. I Math. 304 (1987), $177-180$.

[Ro5] _ Intersection theorems, Commutative Algebra, Math. Sci. Res. Inst. Publ. 15, Springer-Verlag, New York, Berlin, and Heidelberg, 1989, pp. 417-436.

[S] J.-P. Serre, Algèbre locale: Multiplicités, Lecture Notes in Math., vol. 11, Springer-Verlag, Berlin, Heidelberg, and New York, 1965.

[SGA4 $\frac{1}{2}$ ] P. Deligne avec la collaboration de J. F. Boutot, A. Grothendieck, L. Illusie, et J. L. Verdier, Cohomologie étale (Sém. Géométrie Algébrique du Bois-Marie $4 \frac{1}{2}$ ), Lecture Notes in Math., vol. 569, Springer-Verlag, Berlin, Heidelberg, and New York, 1977.

[Sk] H. Skoda, Applications des techniques $L^{2}$ a la théorie des idéaux d'une algèbre de fonctions holomorphes avec poids, Ann. Sci. Ecole Norm. Sup. (4) 5 (1972), 545-579.

[Sm1] K. E. Smith, Tight closure of parameter ideals and F-rationality, Thesis, Univ. of Michigan, 1993.

[Sm2] _ Tight closure of parameter ideals, Invent. Math. 115 (1994), 41-60.

[Sm3] _ F-rational rings have rational singularities, preprint.

[Sw1] I. Swanson, Joint reductions, tight closure, and the Briançon-Skoda theorem, J. Algebra 147 (1992), 128-136.

[Sw2] _ Tight closure, joint reductions, and mixed multiplicities, Thesis, Purdue Univ., 1992.

[Vel1] J. Velez, Openness of the F-rational locus, smooth base change, and Koh's conjecture, Thesis, Univ. of Michigan, 1993.

[Vel2] Openness of the F-rational locus and smooth base change, J. Algebra (to appear).

[W1] K.-I. Watanabe, Study of F-purity in dimension two, Algebraic Geometry and Commutative Algebra in Honor of Masayoshi Nagata, Vol. II, Kinokuniya, Tokyo, 1988, pp. 791-800.

[W2] _ F-regular and F-pure normal graded rings, J. Pure Appl. Algebra 71 (1991), $341-350$

[WITO] K. Watanabe, T. Ishikawa, S. Tachibana, and K. Otsuka, On tensor products Gorenstein rings, J. Math. Kyoto Univ. 9-3 (1969), 413-423.

[Wil1] L. Williams, Uniform stability of kernels of Koszul cohomology indexed by the Frobenius endomorphism, Thesis, Univ. of Michigan, 1992.

[Wil2] _ Uniform stability of kernels of Koszul cohomology indexed by the Frobenius endomorphism, J. Algebra (to appear).

Department of Mathematics, University of Michigan, Ann Arbor, Michigan 481091003

E-mail address: hochster@math.lsa.umich.edu

Department of Mathematics, Purdue University, West Lafayette, Indiana 47907

E-mail address: huneke@math.purdue.edu 